Supporting Information

\title{
Nickel/Molybdenum Bimetallic Alloy for Dry Reforming of Methane:A Coverage-Dependence Microkinetic Model Simulation Based on the First-Principles Calculation
}

Wan-Ying Wang, Gui-Chang Wang*

(Key Laboratory of Advanced Energy Materials Chemistry (Ministry of Education) and the Tianjin key Lab and Molecule-based Material Chemistry, College of Chemistry, Nankai University, Tianjin 300071, China)

*Corresponding author: Gui-Chang Wang. E-mail: wangguichang@nankai.edu.cn

Telephone: +86-22-23503824(O) Fax: +86-22-23502458 


\section{Part S1 Computational details}

\subsection{DFT calculations}

All DFT calculations in this study are performed by the Vienna ab initio simulation package (VASP). ${ }^{1-3}$ The core-valence electron interaction was represented by the projector augmented wave method (PAW). ${ }^{4} 5$ The exchange and correlation energy were calculated by the Perdew-Burke-Ernzerhof (PBE) ${ }^{6}$ functional within the generalized gradient approximation (GGA). ${ }^{7}$ All calculations were performed with a plane wave cut-off energy of $400 \mathrm{eV}$ and a k-points sampling of $3 \times 3 \times 1$ with Monkhorst-Pack scheme. ${ }^{8} \mathrm{~A}$ force tolerance of $0.05 \mathrm{eV} \AA^{-1}$ were employed to ensure high level of accuracy. Since the system contains magnetic elements, all calculations were considered the spin polarization effect. ${ }^{9}$ For gas molecules that interact weakly with the catalyst surface, their adsorption energies are all corrected by van der Waals using the DFT-D3 method. ${ }^{10}$ The adsorption of CO was corrected using rPBE functional. ${ }^{11}$ The nudged elastic band (CI-NEB) ${ }^{12}$ method was employed to locate the transition states (TSs) and the frequency analysis of the transition state structure is carried out to verify the correctness of the transition state structure.

The optimized lattice constant of unit cell volume of $\mathrm{Ni}$ is $\mathrm{a}_{0}=3.52 \AA$ and the optimized lattice constant of unit cell volume of $\mathrm{MoNi}_{4}$ is $\mathrm{a}_{0}=\mathrm{b}_{0}=5.72 \AA, \mathrm{c}_{0}=3.56 \AA$, which is close to the experimental data. ${ }^{13}$ The surface structure of $\mathrm{Ni}(111)$ consists of a 4-layer slab of $\mathrm{p}(3 \times 3)$ supercell with a $15 \AA$ vacuum separation. The surface structures of $\mathrm{MoNi}_{4}(001)$ and $\mathrm{MoNi}_{3}(100)$ consist of a 4-layer slab of $\mathrm{p}(2 \times 2)$ supercell with a $15 \AA$ vacuum separation. When calculating the adsorption energy and transition state, the bottom two layers of catalyst atoms are fixed, and when calculating the frequency, all the catalyst atoms are fixed.

The formula of adsorption energy $\left(E_{a d s}\right)$, desorption energy $\left(E_{d e s}\right)$, forward reaction activation energy $\left(E_{a}\right)$, reverse reaction activation energy $\left(E_{a}^{-l}\right)$ and reaction energies $(\Delta E)$ were calculated as follows:

$$
\begin{gathered}
E_{\text {ads }}=E_{\text {system }}-E_{\text {surface }}-E_{\text {absorbate }} \\
E_{\text {des }}=E_{\text {surface }}+E_{\text {absorbate }}-E_{\text {system }} \\
E_{a}=E_{T S}-E_{I S} \\
\Delta E=E_{F S}-E_{I S} \\
E_{a}^{-1}=E_{a}-\Delta E=E_{T S}-E_{F S}
\end{gathered}
$$

here, $E_{\text {system }}, E_{\text {surface }}, E_{\text {adsorbate }}, E_{I S}, E_{T S}$, and $E_{F S}$ represent the energy of the adsorption system, surface, adsorbate, initial state (IS), transition state (TS), and final state (FS), respectively.

\subsection{Thermodynamics}

In order to compare to the experimental condition more exactly, we calculated the Gibbs free energy of each species at $1073.15 \mathrm{~K}$ and 1 bar. In all calculations, we ignored the enthalpy correction, because we thought that this part of the energy has a small effect and can be ignored. In other words, the Gibbs free energy of species A at temperature $T$ and pressure $P$ is:

$$
G_{A}(T, P)=E_{\text {total }, A}+E_{Z P E}-T S(T, P)
$$

where $E_{\text {total }, A}$ is the total energy determined by DFT calculations, $E_{Z P E}$ is the zero-point energy and $S$ is the entropy of species $\mathrm{A}$ at that temperature and pressure.

The zero-point energies contributions of all species were considered in our calculations. The zero-point energies can be obtained by the following formula:

$$
E_{Z P E}=\sum_{i}\left(\frac{1}{2}\right) h v_{i}
$$

where $\mathrm{h}$ is Plank's constant and $v_{i}$ is the frequency of the normal mode.

The Gibbs free energy $(\mathrm{G})$ of gas phase species was taken from experimental data directly. ${ }^{14}$ For the adsorption of gas molecules, its adsorption entropy was estimated by using the following formula: ${ }^{15}$

$$
S_{a d s}(\mathrm{~T})=0.7 S_{\text {gas }}(\mathrm{T})-3.3 R
$$

where $S_{a d s}$ is the entropy of the adsorbate, $S_{\text {gas }}$ is the entropy of the corresponding gas phase material obtained from 
the thermodynamic database, and $R$ is the ideal gas constant.

The entropy of all strongly adsorbed species (including the transition state) can be obtained from their vibration frequencies. The contribution of translational and rotational entropy is neglected in the calculation of the entropy of strongly adsorbed species. The vibration entropy can be obtained by the following formula:

$$
S_{v i b}=\sum_{i}^{N}\left[-k_{B} \ln \left(1-e^{\frac{-h v_{i}}{k_{B} T}}\right)-\frac{h v_{i}}{T\left(1-e^{\frac{h v_{i}}{k_{B} T}}\right)}\right]
$$

where $k_{B}$ is Boltzmann's constant.

We plot the (free) energy profiles of elementary reaction or a series of elementary reactions. We used (free) energy barriers to describe the degree of difficulty of elementary reaction or a series of elementary reactions. (Free) Energy barriers mean the difference between the (free) energy of the starting reactant and the (free) energy of the highest transition state.

1.3. Microkinetic model simulation

Microkinetic simulation can provide the reaction rate of each elementary reaction and the coverage of each intermediate species on the catalyst surface under actual reaction conditions. The simulation results are based on the fact that all elementary reactions can be carried out on the surface of the catalyst at the same time, which is very different from the analysis of DFT results based on the sequential order of reactions.

Our microkinetic model was made a series of assumptions: All sites are equivalent; Each site can only adsorb an adsorbed specie; There is no lateral interaction force between adsorbed species; The reactant and product pressures remain constant during the simulation.

Based on the above considerations, our microkinetic model was considered 19 elementary reactions and 4 adsorption/desorption processes. These elementary reaction equations are following:

$$
\begin{aligned}
& \mathrm{CH}_{4} *+* \underset{k_{1 \_r}}{\stackrel{k_{1}}{\rightleftarrows}} \mathrm{CH}_{3} *+H^{*} \\
& \mathrm{CH}_{3} *+* \underset{k_{2-r}}{\stackrel{k_{2}}{\rightleftarrows}} \mathrm{CH}_{2} *+H^{*} \\
& \mathrm{CH}_{2}{ }^{*}+\underset{k_{3-r}}{\stackrel{k_{3}}{\rightleftarrows}} \mathrm{CH} H^{*}+H^{*} \\
& C H^{*}+* \underset{k_{4 \_}}{\stackrel{k_{4}}{\rightleftarrows}} C H^{*}+H^{*} \\
& \mathrm{CO}_{2} *+* \underset{k_{s_{-}}}{\stackrel{k_{5}}{\rightleftarrows}} \mathrm{CO} *+\mathrm{O}^{*} \\
& \mathrm{CO}_{2}{ }^{*}+\mathrm{H}^{*} \underset{k_{6-r}}{\stackrel{k_{6}}{\rightleftarrows}} \mathrm{COOH}^{*}+* \\
& \mathrm{COOH}^{*}+* \underset{k_{7_{-}}}{\stackrel{k_{7}}{\rightleftarrows}} \mathrm{CO}^{*}+\mathrm{OH}^{*} \\
& \mathrm{CH}_{3}{ }^{*}+\mathrm{O}^{*} \underset{k_{8_{-} r}}{\stackrel{k_{8}}{\rightleftarrows}} \mathrm{CH}_{3} \mathrm{O}^{*}+* \\
& \mathrm{CH}_{3} \mathrm{O}^{*}+* \underset{k_{9-r}}{\stackrel{k_{9}}{\rightleftarrows}} \mathrm{CH}_{2} \mathrm{O}^{*}+\mathrm{H}^{*} \\
& \mathrm{CH}_{2} *+\mathrm{O} * \underset{k_{10} r}{\stackrel{k_{10}}{\rightleftarrows}} \mathrm{CH}_{2} \mathrm{O} *+ \\
& \mathrm{CH}_{2} \mathrm{O} *+* \underset{k_{11 \_r}}{\stackrel{k_{11}}{\rightleftarrows}} \mathrm{CHO} *+\mathrm{H}^{*} \\
& \mathrm{CH}^{*}+\mathrm{O}^{*} \underset{k_{12 \_r}}{\stackrel{k_{12}}{\rightleftarrows}} \mathrm{CHO} \mathrm{H}^{*} \text { * }
\end{aligned}
$$




$$
\begin{aligned}
& C H O^{*}+* \underset{k_{13} r}{\stackrel{k_{13}}{\rightleftarrows}} C O^{*}+H^{*} \\
& C^{*}+O^{*} \underset{k_{14 \_r}}{\stackrel{k_{14}}{\rightleftarrows}} C O^{*}+* \\
& 2 H * \underset{k_{15 \_r}}{\stackrel{k_{15}}{\rightleftarrows}} H_{2}(g)+2 * \\
& O^{*}+H^{*} \underset{k_{16 \_}}{\stackrel{k_{16}}{\rightleftarrows}} O H^{*}+* \\
& O H^{*}+H^{*} \underset{k_{17 \_} r}{\stackrel{k_{17}}{\rightleftarrows}} H_{2} O^{*}+* \\
& 2 C O * \underset{k_{18 \_r}}{\stackrel{k_{18}}{\rightleftarrows}} C O_{2}{ }^{*}+C^{*} \\
& 2 \mathrm{OH} * \underset{k_{19 \_} r}{\stackrel{k_{19}}{\rightleftarrows}} H_{2} O^{*}+O^{*} \\
& C O * \underset{k_{20} r}{\stackrel{k_{20}}{\rightleftarrows}} C O(g)+* \\
& \mathrm{CO}_{2}(g)+* \underset{k_{21 \_r}}{\stackrel{k_{21}}{\rightleftarrows}} \mathrm{CO}_{2} * \\
& \mathrm{H}_{2} \mathrm{O} * \underset{k_{22}-r}{\stackrel{k_{22}}{\rightleftarrows}} H_{2} \mathrm{O}(\mathrm{g})+* \\
& \mathrm{CH}_{4}(\mathrm{~g})+* * \underset{k_{23}}{\stackrel{k_{23} r}{\rightleftarrows}} \mathrm{CH}_{4} *
\end{aligned}
$$

where * represents the vacancy on the surface of the catalyst, $k_{i}$ and $k_{i_{-} r}$ are the rate constants of the forward and reverse reactions.

The rate constant of adsorption process was treated by the general collision theory: ${ }^{16}$

$$
k_{a d s}=\frac{A_{s i t e} P_{i} \sigma_{i}}{\sqrt{2 \pi m_{i} k_{B} T}} \exp \left(-\frac{E_{a}}{R T}\right)=A_{a d s}^{0} P_{i} \exp \left(-\frac{E_{a}}{R T}\right)
$$

where $A_{\text {site, }}, P_{i}, \sigma_{i}, m_{i}, k_{B}, T, E_{a}$ and $A_{a d s}^{0}$ refer to the area of the adsorption site, the partial pressure, the sticking probability, the mass of component $i$, the Boltzmann constant, the reaction temperature, activation energy and the pre-exponential factor of adsorption process, respectively. For the adsorption process, as the reaction progresses, the active sites on the surface decrease, which in turn reduces the corresponding sticking probability. Therefore, we assume that the initial sticking probability is the square of surface active site *. In the current study, the initial viscosity coefficient is set to 1 . The rate constant of the desorption process was calculated by the following formula: ${ }^{17}$

$$
k_{\text {des }}=\frac{k_{B} T^{3}}{h^{3}} \frac{A_{\text {site }}\left(2 \pi m_{i} k_{B}\right)}{\sigma \theta_{\text {rot }}} \exp \left(-\frac{E_{d e s}}{k_{B} T}\right)=A_{d e s}^{0} \exp \left(-\frac{E_{d e s}}{k_{B} T}\right)
$$

where $\sigma$ is the (dimensionless) symmetry number, $\theta$ is the characteristic temperature for rotation, $E_{d e s}$ is the desorption energy, and $A_{d e s}^{0}$ is the pre-exponential factor of desorption process. For the adsorption process, its barrier was assumed to be zero due to the non-activated event. For the desorption process, its barrier was set to be the negative magnitude of related adsorption energy. For the surface reaction process, its rate constant was predicated according to the transition state theory:

$$
\mathrm{k}=\frac{k_{B} T}{h} \exp \left(\frac{-\Delta G}{k_{B} T}\right)=A^{0} \exp \left(\frac{-\Delta G}{k_{B} T}\right)
$$

Correspondingly, the reaction rate of each elementary step in the microkinetic model is expressed as:

$$
\begin{aligned}
& r_{1}=k_{1} \theta_{C_{4}{ }^{*}} \theta_{*}-k_{11_{-} r} \theta_{\mathrm{CH}_{3}{ }^{*}} \theta_{H^{*}} \\
& r_{2}=k_{2} \theta_{\mathrm{CH}_{3}{ }^{*}} \theta_{*}-k_{2{ }_{2} r} \theta_{\mathrm{CH}_{2}{ }^{*}} \theta_{H^{*}}
\end{aligned}
$$




$$
\begin{aligned}
& r_{3}=k_{3} \theta_{\mathrm{CH}_{2}}{ }^{*} \theta_{*}-k_{3 \_}{ }_{-} \theta_{C H^{*}} \theta_{H^{*}} \\
& r_{4}=k_{4} \theta_{C H^{*}} \theta_{*}-k_{4-r} \theta_{C^{*}} \theta_{H^{*}} \\
& r_{5}=k_{5} \theta_{\mathrm{CO}_{2}{ }^{*}} \theta_{*}-k_{5-r} \theta_{\mathrm{CO}^{*}} \theta_{O^{*}} \\
& r_{6}=k_{6} \theta_{\mathrm{CO}_{2}} \theta_{H^{*}}-k_{6 \_r} \theta_{\mathrm{COOH}^{*}} \theta_{*} \\
& r_{7}=k_{7} \theta_{\mathrm{COOH}^{*}} \theta_{*}-k_{7 \_r} \theta_{\mathrm{CO}^{*}} \theta_{\mathrm{OH}^{*}} \\
& r_{8}=k_{8} \theta_{C_{3}{ }_{3}} \theta_{O^{*}}-k_{8 \_}{ }_{-r} \theta_{C_{3} O^{*}} \theta_{*} \\
& r_{9}=k_{9} \theta_{\mathrm{CH}_{3} \mathrm{O}^{*}} \theta_{*}-k_{9{ }_{-} r} \theta_{\mathrm{CH}_{2} \mathrm{O}^{*}} \theta_{H^{*}} \\
& r_{10}=k_{10} \theta_{\mathrm{CH}_{2} *} \theta_{\mathrm{O}^{*}}-k_{10 \_r} \theta_{\mathrm{CH}_{2} \mathrm{O}^{*}} \theta_{*} \\
& r_{11}=k_{11} \theta_{\mathrm{CH}_{2} \mathrm{O}^{*}} \theta_{*}-k_{11{ }_{-} r} \theta_{\mathrm{CHO}^{*}} \theta_{H^{*}} \\
& r_{12}=k_{12} \theta_{C H^{*}} \theta_{O^{*}}-k_{12 \_} r \theta_{C H O^{*}} \theta_{*} \\
& r_{13}=k_{13} \theta_{C H O^{*}} \theta_{*}-k_{13 \_r} \theta_{C O^{*}} \theta_{H^{*}} \\
& r_{14}=k_{14} \theta_{C^{*}} \theta_{O^{*}}-k_{14 \_r} \theta_{C O^{*}} \theta_{*} \\
& r_{15}=k_{15} \theta_{H^{*}} \theta_{H^{*}}-k_{15_{-} r} \theta_{*} \theta_{*} P\left(H_{2}\right) \\
& r_{16}=k_{16} \theta_{O^{*}} \theta_{H^{*}}-k_{16 \_r} \theta_{O H} \theta_{*} \\
& r_{17}=k_{17} \theta_{\mathrm{OH}^{*}} \theta_{H^{*}}-k_{17 \_} \theta_{\mathrm{H}_{2} \mathrm{O}^{*}} \theta_{*} \\
& r_{18}=k_{18} \theta_{C O^{*}} \theta_{C O^{*}}-k_{18 \_} r \theta_{\mathrm{CO}_{2} *} \theta_{C^{*}} \\
& r_{19}=k_{19} \theta_{\mathrm{OH}^{*}} \theta_{\mathrm{OH}^{*}}-k_{19 \_r} \theta_{\mathrm{H}_{2} \mathrm{O}^{*}} \theta_{\mathrm{O}^{*}} \\
& r_{20}=k_{20} \theta_{C O^{*}}-k_{20 r} \theta_{*} P(C O) \\
& r_{21}=k_{21} \theta_{*} P\left(C O_{2}\right)-k_{21}{ }_{r} \theta_{C_{2}{ }^{*}} \\
& r_{22}=k_{22} \theta_{H_{2} O^{*}}-k_{22}{ }_{r} \theta_{*} P\left(H_{2} \mathrm{O}\right) \\
& r_{23}=k_{23} \theta_{*} P\left(\mathrm{CH}_{4}\right)-k_{23_{-} r} \theta_{\mathrm{CH}_{4}{ }^{*}}
\end{aligned}
$$

where $\theta_{i *}$ represents the coverage of specie $\mathrm{i}$ on the catalyst surface. A total of 16 species (including vacancy) coverage are included in our model.

The rate of change of species coverage over time is affected by the reaction rate. In our model, the rate of change of the coverage of each species over time is expressed as:

$$
\begin{gathered}
\frac{d}{d t} \theta_{*}=-r 1-r 2-r 3-r 4-r 5+r 6-r 7+r 8-r 9+r 10-r 11+r 12-r 13+r 14+2 * r 15+r 16+r 17+r 20-r 21+r 22-r 23 \\
\frac{d}{d t} \theta_{\mathrm{CH}_{3}{ }^{*}}=r 1-r 2-r 8 \\
\frac{d}{d t} \theta_{H^{*}}=r 1+r 2+r 3+r 4-r 6+r 9+r 11+r 13-2 * r 15-r 16-r 17 \\
\frac{d}{d t} \theta_{\mathrm{CH}_{2}^{*}}=r 2-r 3-r 10 \\
\frac{d}{d t} \theta_{\mathrm{CH}^{*}}=r 3-r 4-r 12 \\
\frac{d}{d t} \theta_{C^{*}}=r 4-r 14+r 18
\end{gathered}
$$




$$
\begin{gathered}
\frac{d}{d t} \theta_{\mathrm{CO}^{*}}=r 5+r 7+r 13+r 14-2 * r 18-r 20 \\
\frac{d}{d t} \theta_{O^{*}}=r 5-r 8-r 10-r 12-r 14-r 16+r 19 \\
\frac{d}{d t} \theta_{\mathrm{COOH}^{*}}=r 6-r 7 \\
\frac{d}{d t} \theta_{\mathrm{OH}^{*}}=r 7+r 16-r 17-2 * r 19 \\
\frac{d}{d t} \theta_{\mathrm{CH}_{3} O^{*}}=r 8-r 9 \\
\frac{d}{d t} \theta_{\mathrm{CH}_{2} \mathrm{O}^{*}}=r 9+r 10-r 11 \\
\frac{d}{d t} \theta_{\mathrm{CHO}^{*}}=r 11+r 12-r 13 \\
\frac{d}{d t} \theta_{\mathrm{CO}^{*}}=-r 5-r 6+r 18+r 21 \\
\frac{d}{d t} \theta_{\mathrm{H}_{2} \mathrm{O}^{*}}=r 17+r 19-r 22 \\
\frac{d}{d t} \theta_{\mathrm{CH}_{4^{*}}}=-r 1+r 23
\end{gathered}
$$

The linear ordinary differential equation was integrated numerically by the ISODA integrator in the ODEPACK ${ }^{18}$ package wrapped in SciPytools, which is able to automatically select methods based on the stiffness of the problem (Adams method for non-stiff problems, and the backward differentiation formula method for stiff cases). ${ }^{19}$

The basic microkinetic model (the above principle) ignores the influence of coverage on the adsorption and reaction process, which is contrary to the actual situation. The actual situation is that the coverage has a great influence on chemical adsorption and reaction. And there are quite a lot of experiments and theoretical calculations to confirm this. Therefore, we additionally consider the impact of coverage. ${ }^{20}$ Here we mainly consider the impact of $\mathrm{O}^{*}$ coverage on the $\mathrm{CO}_{2} *$ activation process to simplify the calculation. This is because $\mathrm{CO}_{2} *$ is very easy to activate on the alloy, making the $\mathrm{O}^{*}$ coverage extremely high. Therefore, the influence of $\mathrm{O}^{*}$ on the $\mathrm{CO}_{2}$ * activation process cannot be easily ignored. The influence of $\mathrm{O}^{*}$ coverage on the activation energy of $\mathrm{CO}_{2} *$ exists in this form:

$$
E_{a}\left(\mathrm{CO}_{2} * \text { activation }\right)=a \theta_{O} *^{2}+b \theta_{O} *+c
$$

where $\theta_{O}$ is the coverage of $\mathrm{O}^{*}$ on the catalyst surface, a, b, and c are the constants calculated by DFT respectively. Although we have greatly simplified the impact of coverage, only considering the impact of $\mathrm{O}^{*}$ coverage on $\mathrm{CO}_{2}$ * activation can still effectively help us understand the impact of surface species coverage on the reaction. 


\section{Part S2 Results and discussions}

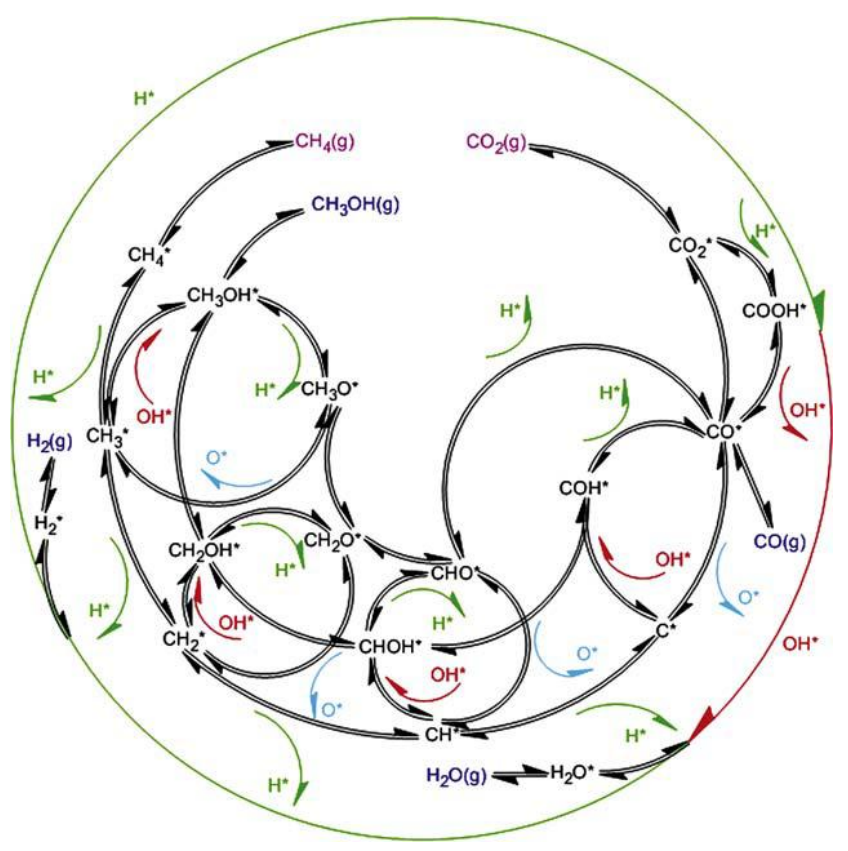

Scheme S1 Complete reaction network for dry reforming reaction. ${ }^{21}$

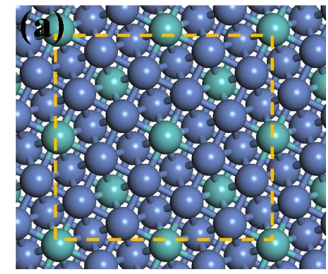

(001)

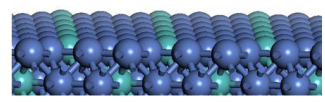

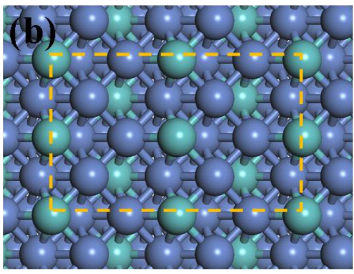

(100)

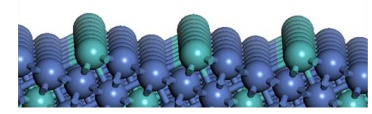

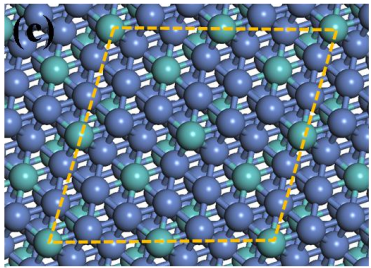

(111)

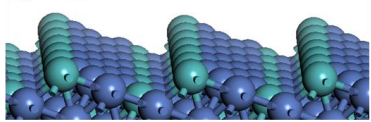

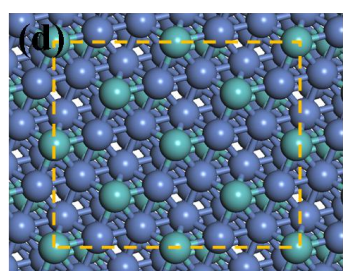

(101)

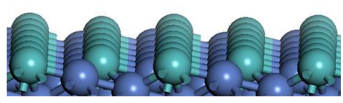

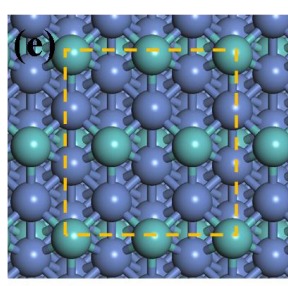

(110)

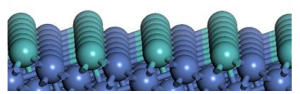

Fig. S1 Top and side views of different crystal planes of $\mathrm{MoNi}_{4}$ alloy. $\mathrm{Ni}$ atoms are represented as blue balls and Mo in cyan. 

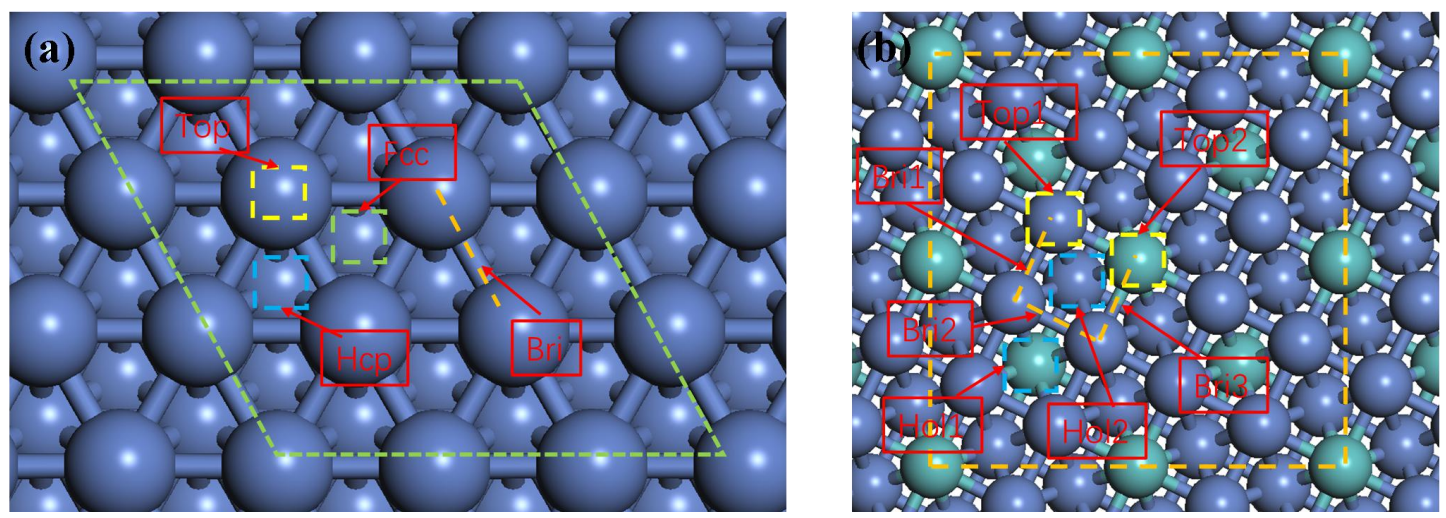

Fig. S2 All possible adsorption sites on $\mathrm{Ni}(111)$ and $\mathrm{MoNi}_{4}(001)$ surfaces. $\mathrm{Ni}$ atoms are represented as blue balls and Mo in cyan.

Table S1 The adsorption energies $\left(E_{a d s}\right)$ and favored adsorption site of the species involved in DRM on Ni(111) and $\mathrm{MoNi}_{4}(001)$.

\begin{tabular}{|c|c|c|c|c|}
\hline \multirow{2}{*}{ Species } & \multicolumn{2}{|c|}{$\mathrm{Ni}(111)$} & \multicolumn{2}{|c|}{$\mathrm{MoNi}_{4}(001)$} \\
\hline & $E_{a d s}(\mathrm{eV})$ & Adsorption site & $E_{a d s}(\mathrm{eV})$ & Adsorption site \\
\hline $\mathrm{CH}_{4} *$ & -0.20 & - & -0.19 & Top2 \\
\hline $\mathrm{CO}_{2} *$ & -0.23 & - & -1.19 & Bri3 \\
\hline $\mathrm{H}_{2} *$ & -0.38 & Top & -0.63 & Top2 \\
\hline $\mathrm{H}_{2} \mathrm{O}^{*}$ & -0.48 & Top & -1.08 & Top2 \\
\hline $\mathrm{CO}^{*}$ & -1.45 & Hcp & -1.45 & Holl \\
\hline $\mathrm{CH}_{3}$ * & -1.95 & Fcc & -2.22 & Top2 \\
\hline $\mathrm{CH}_{2} *$ & -4.12 & Fcc & -4.67 & Hol2 \\
\hline $\mathrm{CH}^{*}$ & -6.50 & Fcc & -7.34 & Hol1 \\
\hline$C^{*}$ & -6.72 & Hep & -8.17 & Hol1 \\
\hline $\mathrm{H}^{*}$ & -2.80 & Fcc & -2.86 & Holl \\
\hline $\mathrm{O}^{*}$ & -5.47 & Fcc & -6.49 & Top2 \\
\hline $\mathrm{OH}^{*}$ & -3.46 & Fcc & -4.30 & Bri3 \\
\hline $\mathrm{CH}_{3} \mathrm{O}^{*}$ & -2.56 & Fcc & -3.57 & Top2 \\
\hline $\mathrm{CH}_{2} \mathrm{O}^{*}$ & -0.55 & Hep & -1.88 & Bri3 \\
\hline $\mathrm{CHO}^{*}$ & -2.24 & Fcc & -3.18 & Hol2 \\
\hline $\mathrm{COOH}^{*}$ & -2.14 & Fec & -3.28 & Hol2 \\
\hline
\end{tabular}

Note: Zero point energy (ZPE) and entropic contributions are not included. 


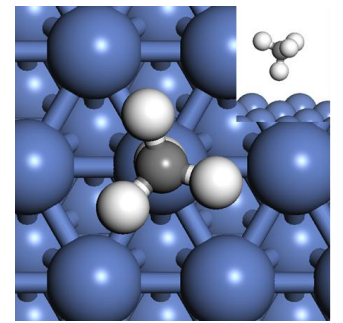

(a) $\mathrm{CH}_{4}$ *

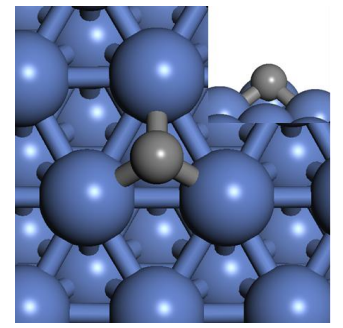

(e) $\mathrm{C}^{*}$

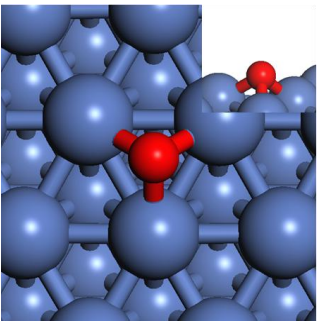

(i) $\mathrm{O}^{*}$

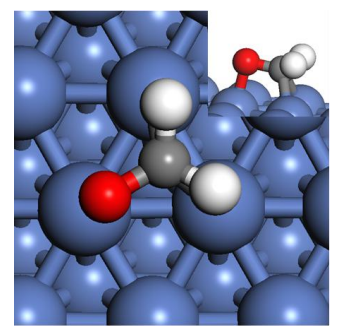

(m) $\mathrm{CH}_{2} \mathrm{O}^{*}$

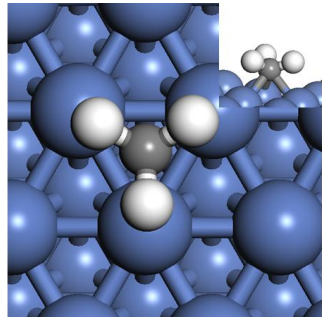

(b) $\mathrm{CH}_{3}$ *

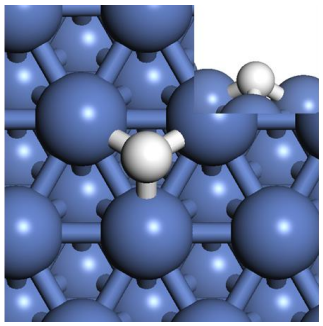

(f) $\mathrm{H}^{*}$

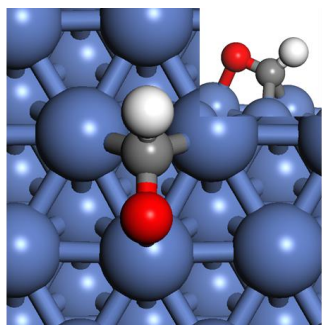

(j) $\mathrm{CHO}^{*}$

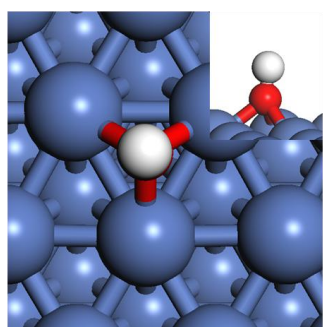

(n) $\mathrm{OH}^{*}$

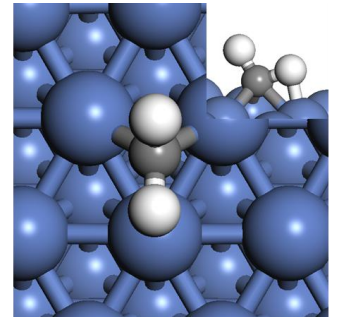

(c) $\mathrm{CH}_{2}$ *

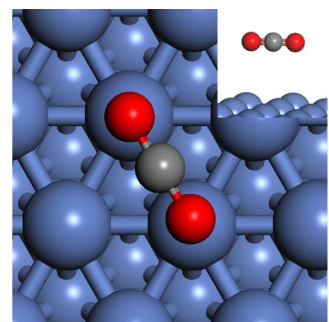

(g) $\mathrm{CO}_{2}{ }^{*}$

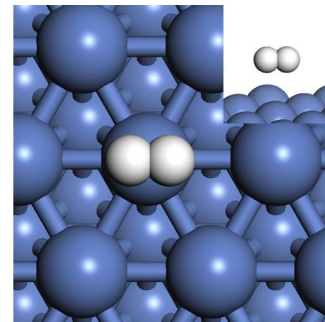

(k) $\mathrm{H}_{2}$ *

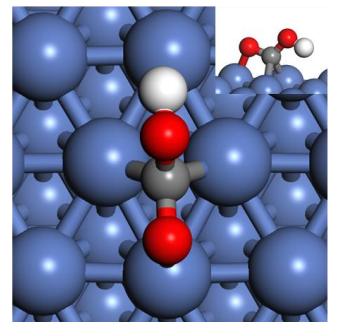

(o) $\mathrm{COOH}^{*}$

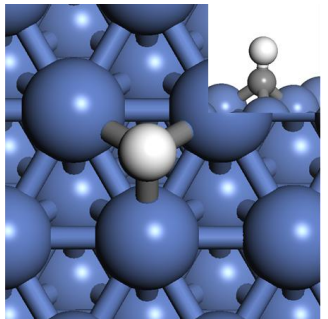

(d) $\mathrm{CH}^{*}$

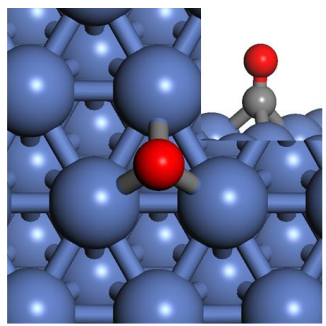

(h) $\mathrm{CO}^{*}$

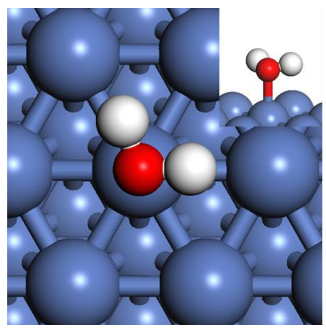

(l) $\mathrm{H}_{2} \mathrm{O}^{*}$

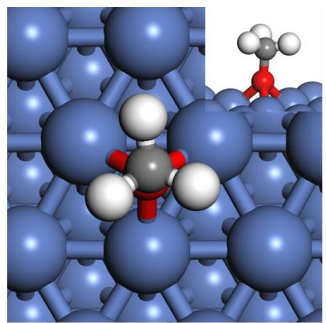

(p) $\mathrm{CH}_{3} \mathrm{O}^{*}$

Fig. S3 The adsorption configurations of the intermediate species involved in the DRM reaction on Ni(111). Ni atoms are represented as blue balls, $\mathrm{C}$ in gray, $\mathrm{O}$ in red and $\mathrm{H}$ in white. 

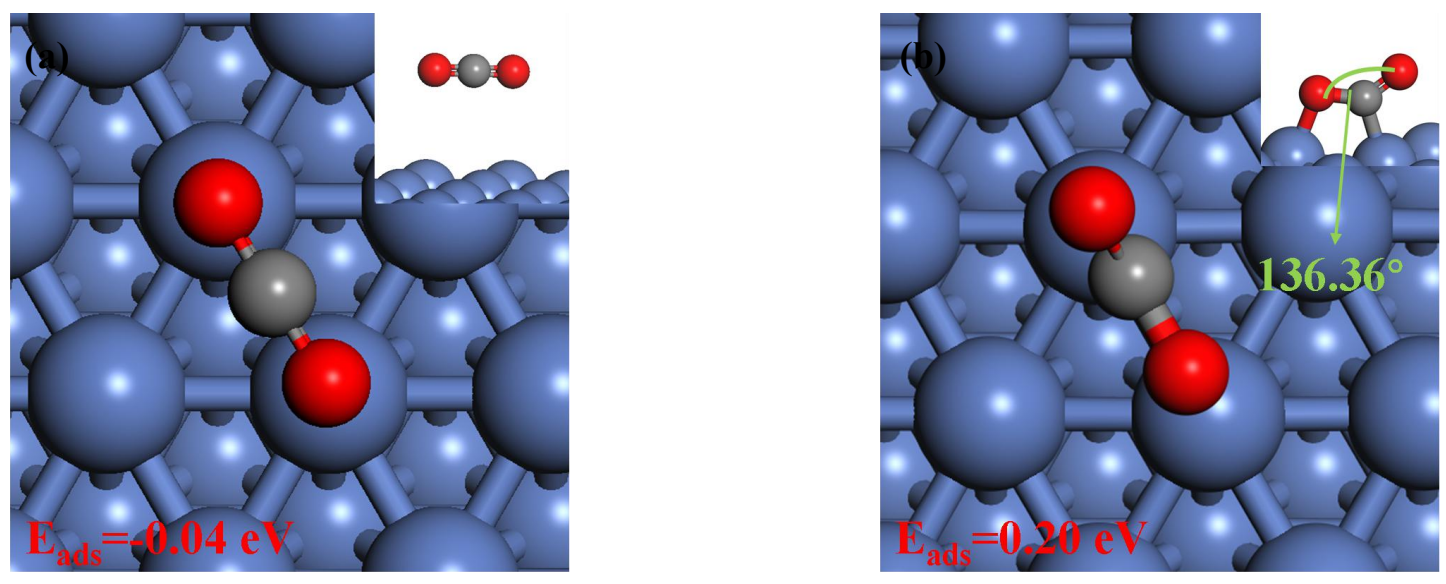

Fig. S4 Two adsorption configurations of $\mathrm{CO}_{2}$ on $\mathrm{Ni}$ (111) : linear (a) and curved (b). The corresponding adsorption energies of the two adsorption configurations are indicated. $\mathrm{Ni}$ atoms are represented as blue balls, $\mathrm{C}$ in gray, $\mathrm{O}$ in red and $\mathrm{H}$ in white.

Table S2 Comparison of adsorption energies of intermediate species on $\mathrm{Ni}$ (111) with previous results. ${ }^{21}$

\begin{tabular}{ccc}
\hline Species & $\mathrm{Ni}(111)$ & Ref. Ni(111) \\
\hline $\mathrm{CH}_{3}{ }^{*}$ & -1.95 & -1.91 \\
$\mathrm{CH}_{2}{ }^{*}$ & -4.12 & -4.01 \\
$\mathrm{CH}^{*}$ & -6.50 & -6.43 \\
$\mathrm{C}^{*}$ & -6.72 & -6.78 \\
$\mathrm{H}^{*}$ & -2.80 & -2.81 \\
$\mathrm{O}^{*}$ & -5.47 & -5.67 \\
$\mathrm{OH}^{*}$ & -3.46 & -3.42 \\
$\mathrm{CH}_{3} \mathrm{O}^{*}$ & -2.56 & -2.63 \\
$\mathrm{CH}_{2} \mathrm{O}^{*}$ & -0.55 & -0.75 \\
$\mathrm{CHO}^{*}$ & -2.24 & -2.26 \\
$\mathrm{COOH}^{*}$ & -2.14 & -2.26 \\
\hline
\end{tabular}

Note: All values are expressed in eV. Zero point energy (ZPE) and entropic contributions are not included. 


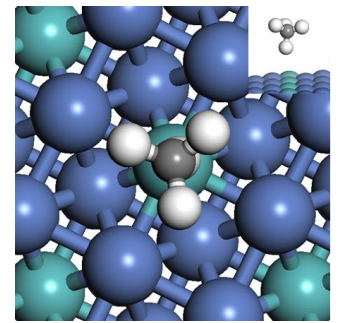

(a) $\mathrm{CH}_{4}$ *

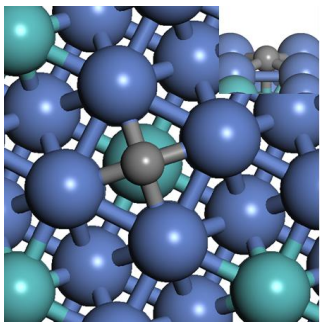

(e) $\mathrm{C}^{*}$

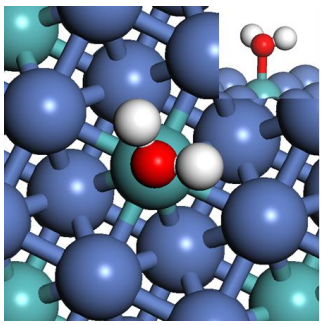

(i) $\mathrm{H}_{2} \mathrm{O}$ *

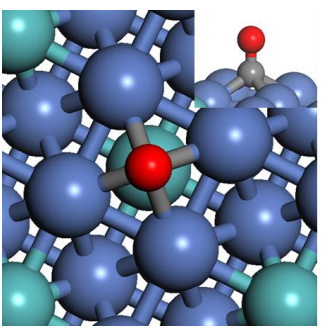

(m) $\mathrm{CO}^{*}$

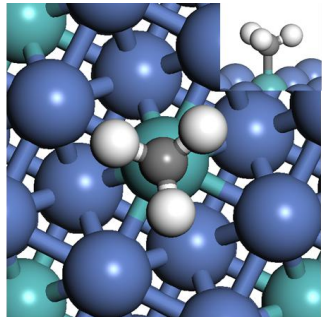

(b) $\mathrm{CH}_{3}$ *

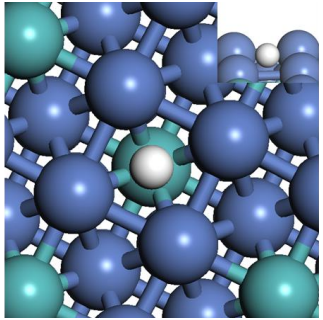

(f) $\mathrm{H}^{*}$

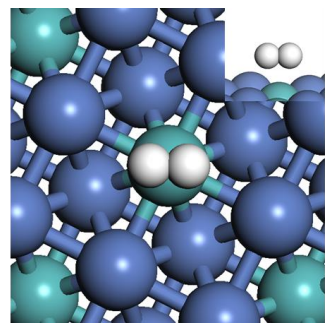

(j) $\mathrm{H}_{2}$ *

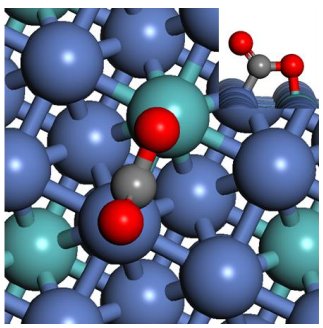

(n) $\mathrm{CO}_{2}^{*}$

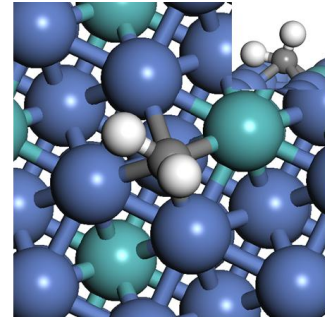

(c) $\mathrm{CH}_{2}$ *

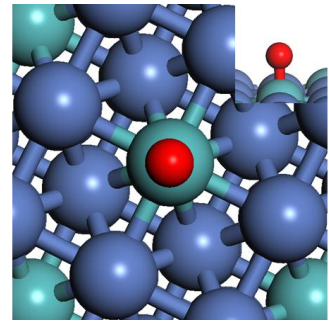

(g) $\mathrm{O}^{*}$

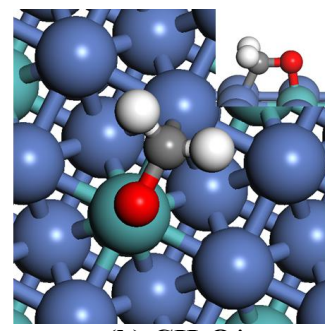

(k) $\mathrm{CH}_{2} \mathrm{O}^{*}$

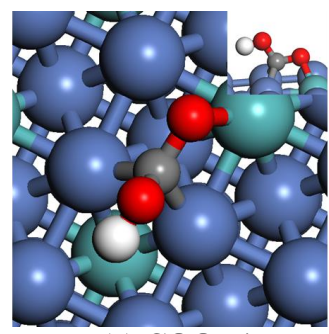

(o) $\mathrm{COOH}^{*}$

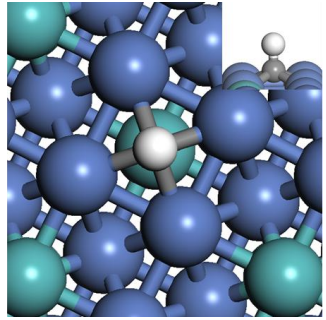

(d) $\mathrm{CH}^{*}$

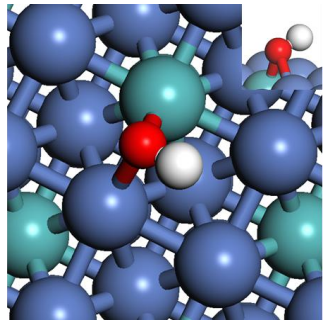

(h) $\mathbf{O H}^{*}$

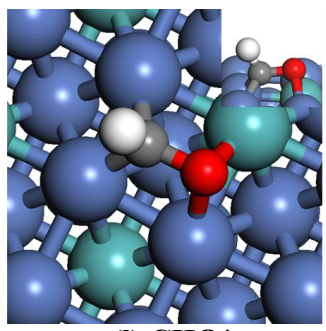

(I) $\mathrm{CHO}^{*}$

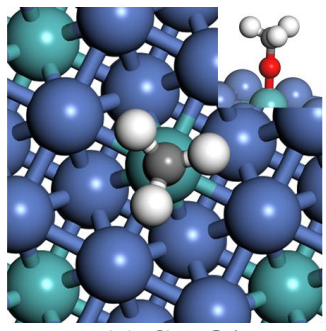

(p) $\mathrm{CH}_{3} \mathrm{O}$ *

Fig. S5 The adsorption configurations of the intermediate species involved in the DRM reaction on $\mathrm{MoNi}_{4}(001)$.

$\mathrm{Ni}$ atoms are represented as blue balls, Mo in cyan, $\mathrm{C}$ in gray, $\mathrm{O}$ in red and $\mathrm{H}$ in white.
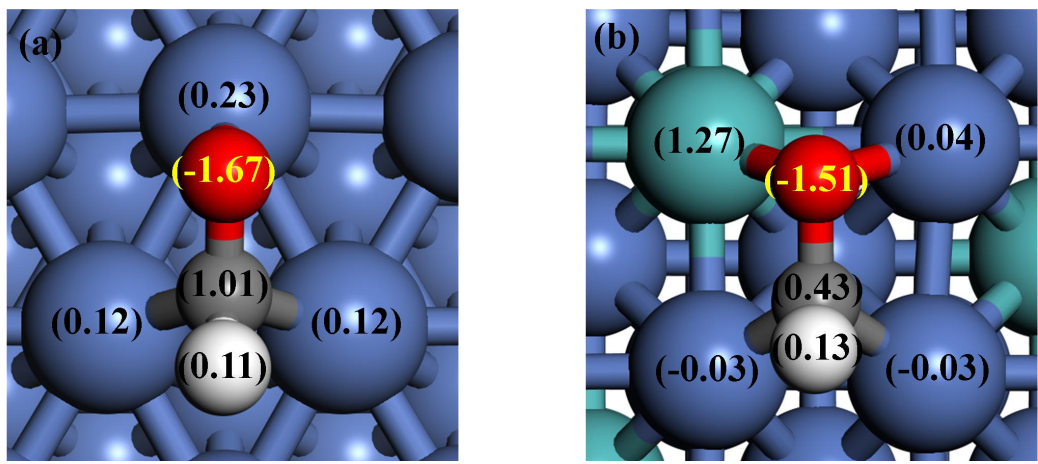

Fig. S6 The bader charge analysis of the adsorption of $\mathrm{CHO}^{*}$ on $\mathrm{Ni}(111)$ (a) and $\mathrm{MoNi}_{4}(001)$ (b) surfaces. The charged state of each atom is shown. $\mathrm{Ni}$ atoms are represented as blue balls, Mo in cyan, $\mathrm{C}$ in gray, $\mathrm{O}$ in red and $\mathrm{H}$ in white. 

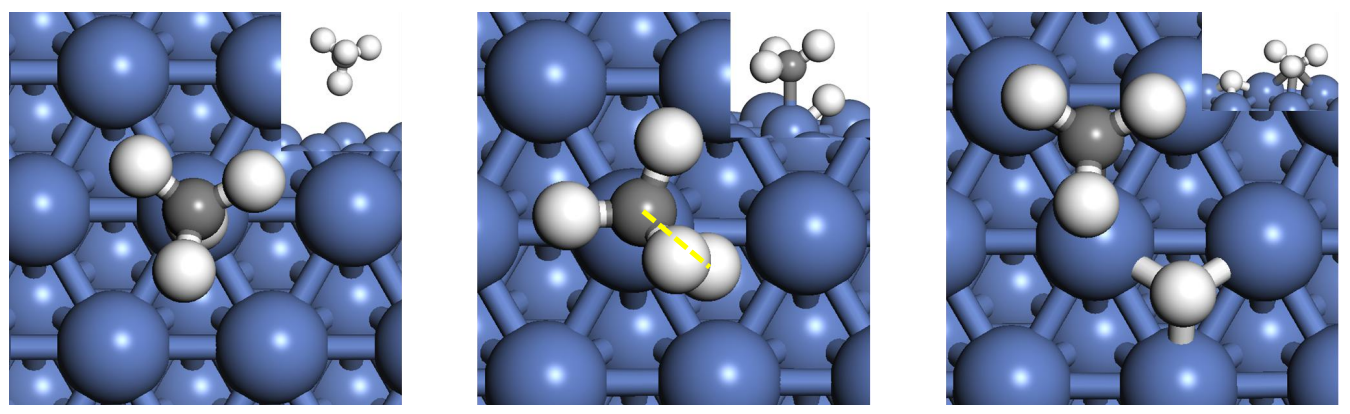

(a) $\mathrm{CH}_{4} *=\mathrm{CH}_{3} *+\mathrm{H}^{*}\left(\mathrm{~d}_{\mathrm{C}-\mathrm{H}}=1.60 \AA\right)$
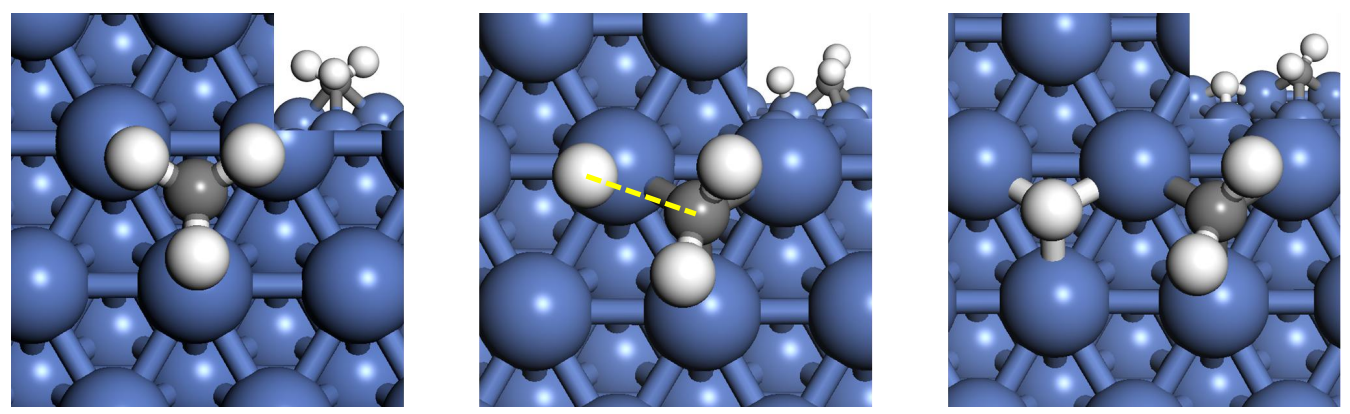

(b) $\mathrm{CH}_{3}{ }^{*}=\mathrm{CH}_{2}{ }^{*}+\mathrm{H}^{*}\left(\mathrm{~d}_{\mathrm{C}-\mathrm{H}}=1.78 \AA\right)$
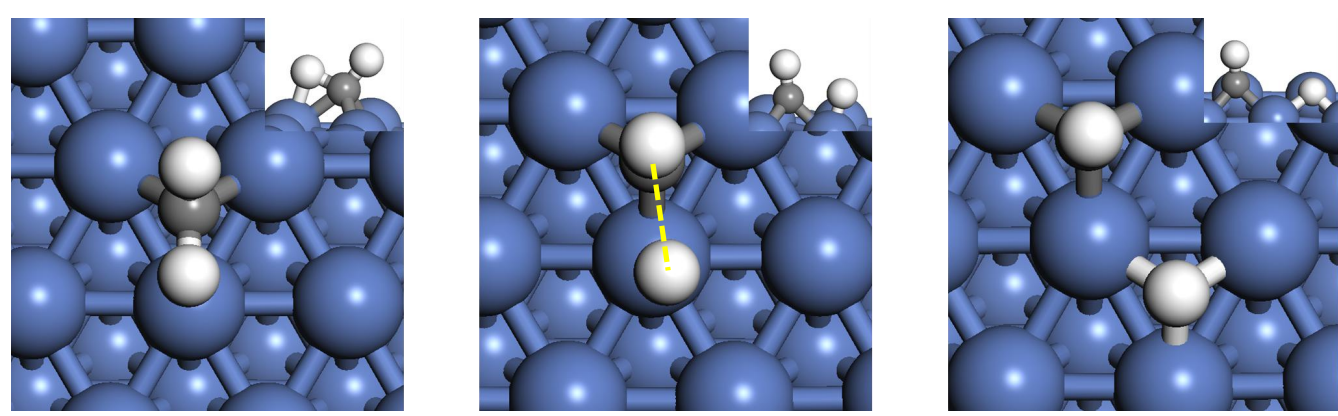

(c) $\mathrm{CH}_{2} *=\mathrm{CH}^{*}+\mathrm{H}^{*}\left(\mathrm{~d}_{\mathrm{C}-\mathrm{H}}=1.73 \AA\right)$
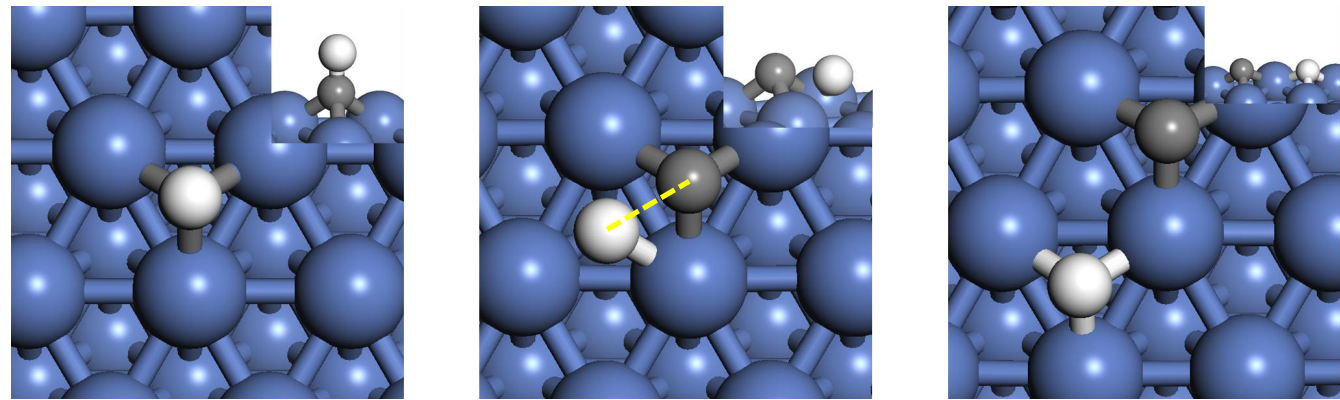

(d) $\mathrm{CH}^{*}=\mathrm{C}^{*}+\mathrm{H}^{*}\left(\mathrm{~d}_{\mathrm{C}-\mathrm{H}}=1.46 \AA\right)$
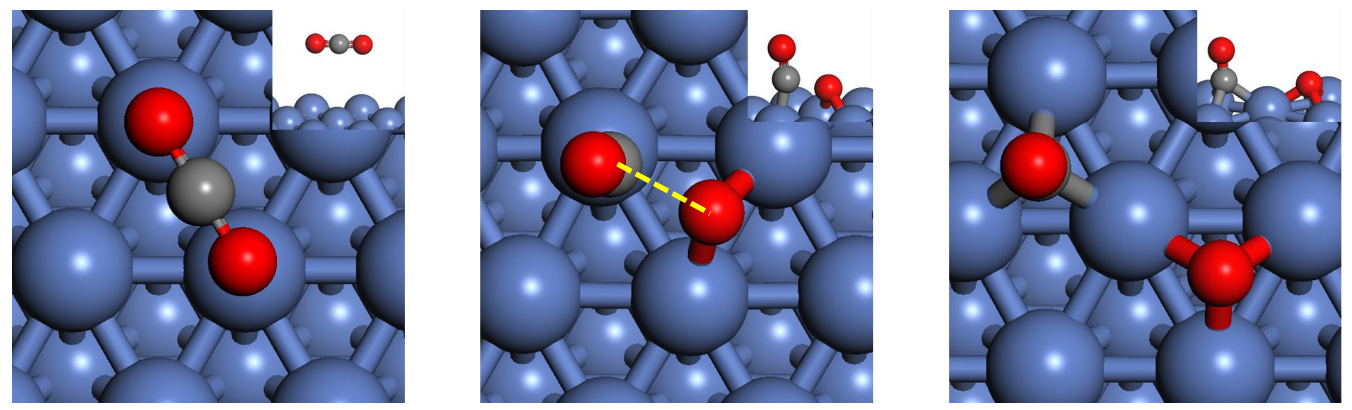

(e) $\mathrm{CO}_{2} *=\mathrm{CO}^{*}+\mathrm{O}^{*}\left(\mathrm{~d}_{\mathrm{C}-\mathrm{O}}=1.64 \AA\right)$ 

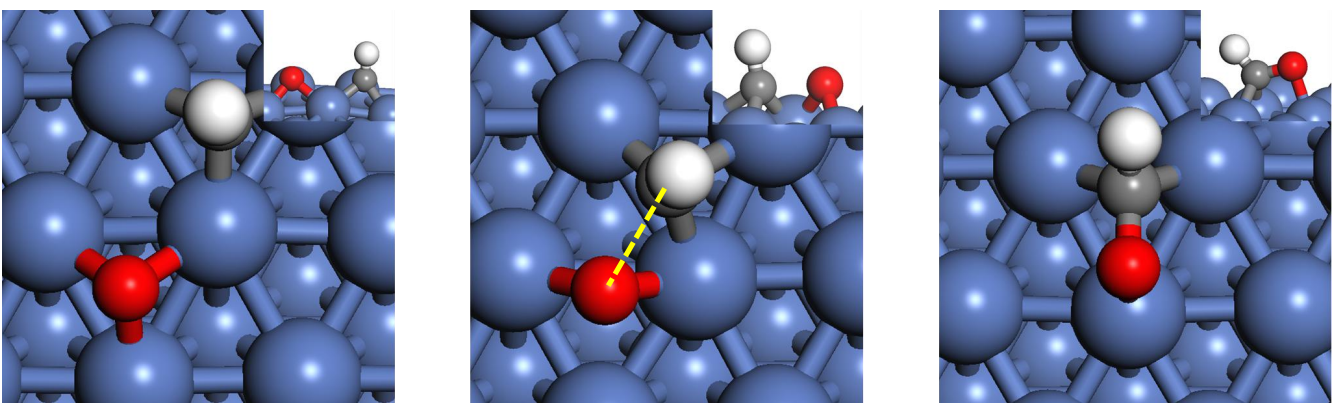

(f) $\mathrm{CH}^{*}+\mathrm{O}^{*}=\mathrm{CHO}^{*}\left(\mathrm{~d}_{\mathrm{C}-\mathrm{O}}=1.81 \AA\right)$
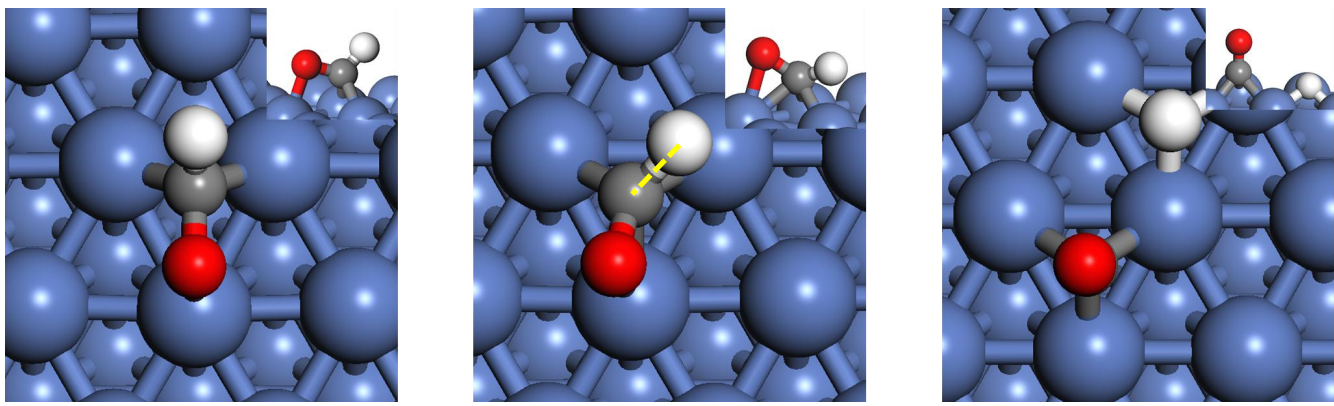

(g) $\mathrm{CHO}^{*}=\mathrm{CO}^{*}+\mathrm{H}^{*}\left(\mathrm{~d}_{\mathrm{C}-\mathrm{H}}=1.16 \AA\right)$
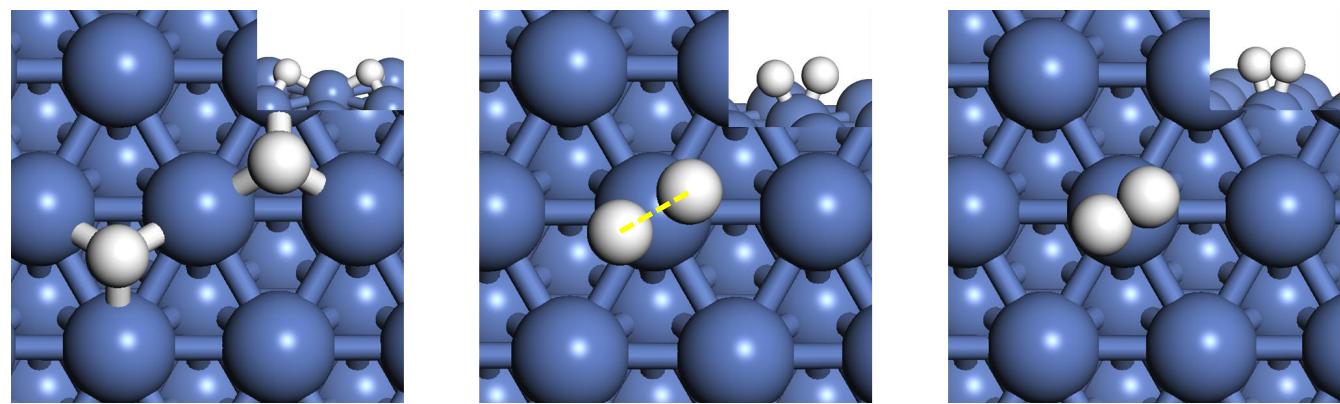

(h) $2 \mathrm{H}^{*}=\mathrm{H}_{2} *\left(\mathrm{~d}_{\mathrm{H}-\mathrm{H}}=1.22 \AA\right)$
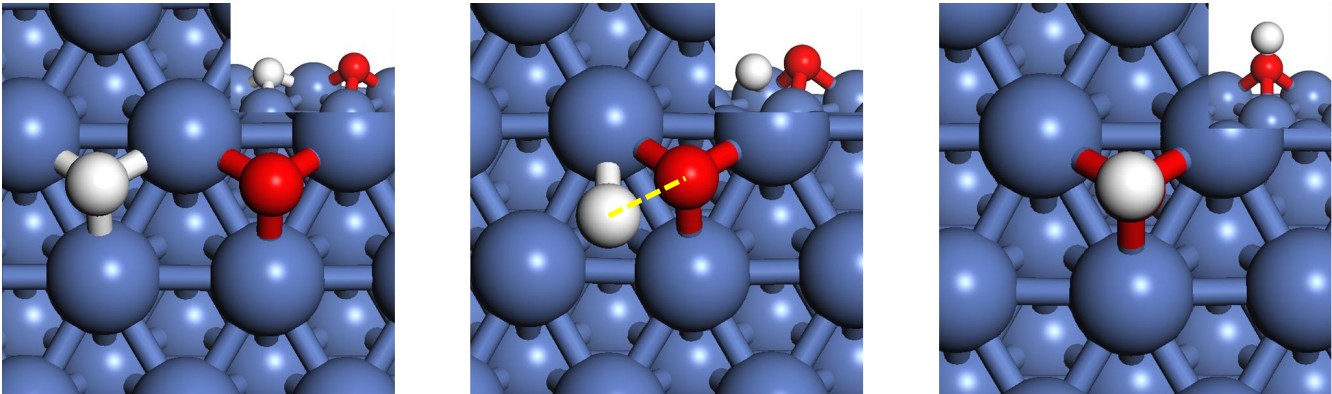

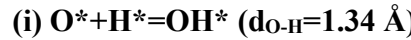
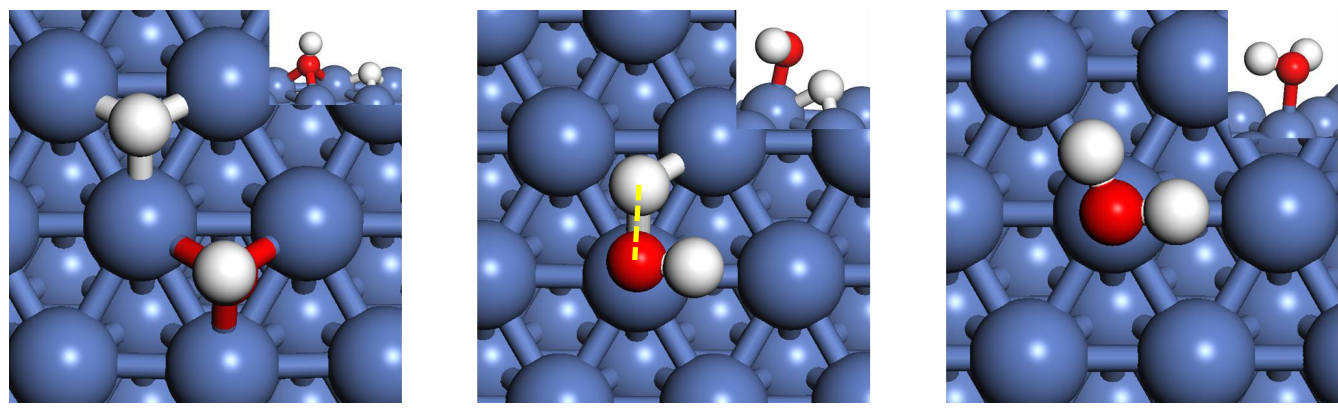

(j) $\mathrm{OH}^{*}+\mathrm{H}^{*}=\mathrm{H}_{2} \mathrm{O}^{*}\left(\mathrm{~d}_{\mathrm{O}-\mathrm{H}}=1.54 \AA\right)$ 

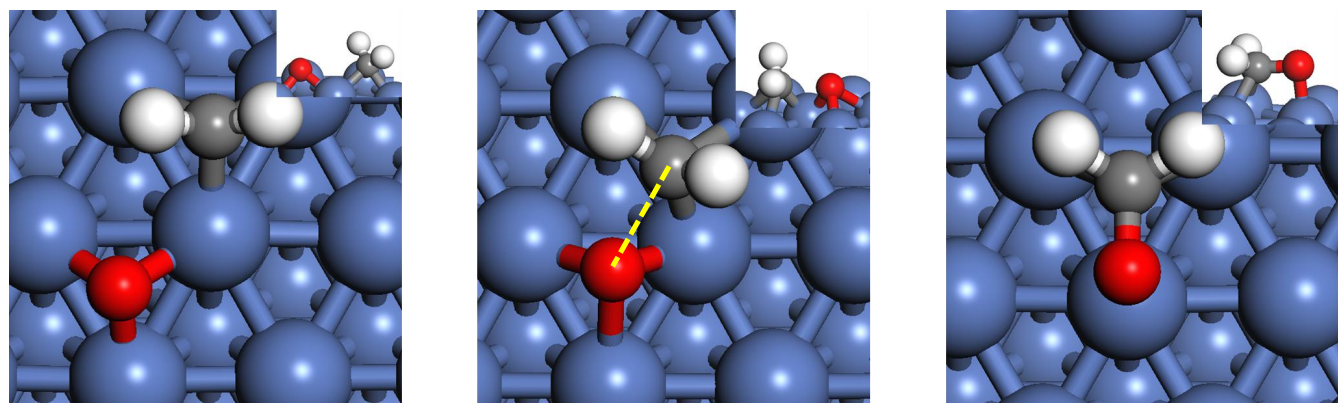

(k) $\mathrm{CH}_{2} *+\mathrm{O} *=\mathrm{CH}_{2} \mathrm{O} *\left(\mathrm{~d}_{\mathrm{C}-\mathrm{O}}=1.82 \AA\right)$
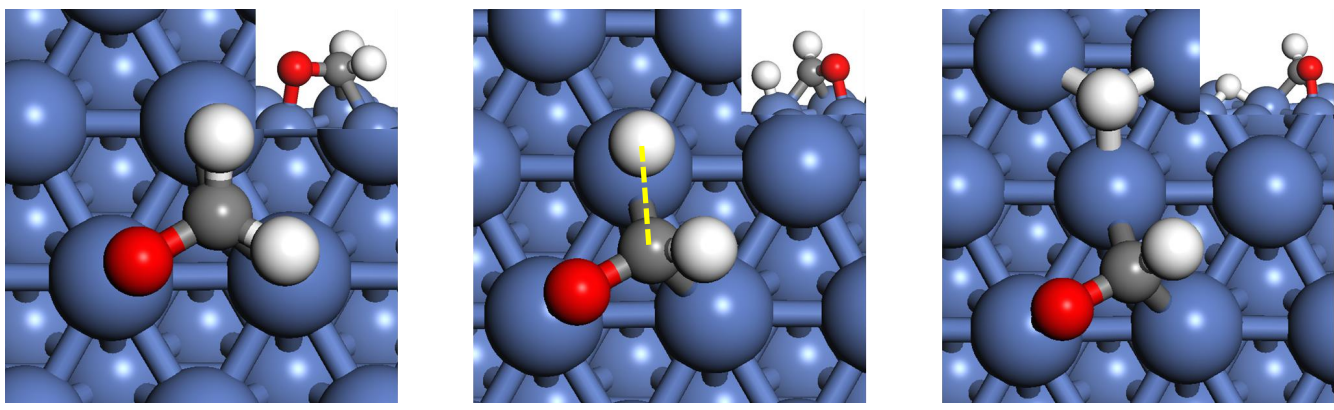

(l) $\mathrm{CH}_{2} \mathrm{O}^{*}=\mathrm{CHO}^{*}+\mathrm{H}^{*}\left(\mathrm{~d}_{\mathrm{C}-\mathrm{H}}=1.59 \AA\right)$
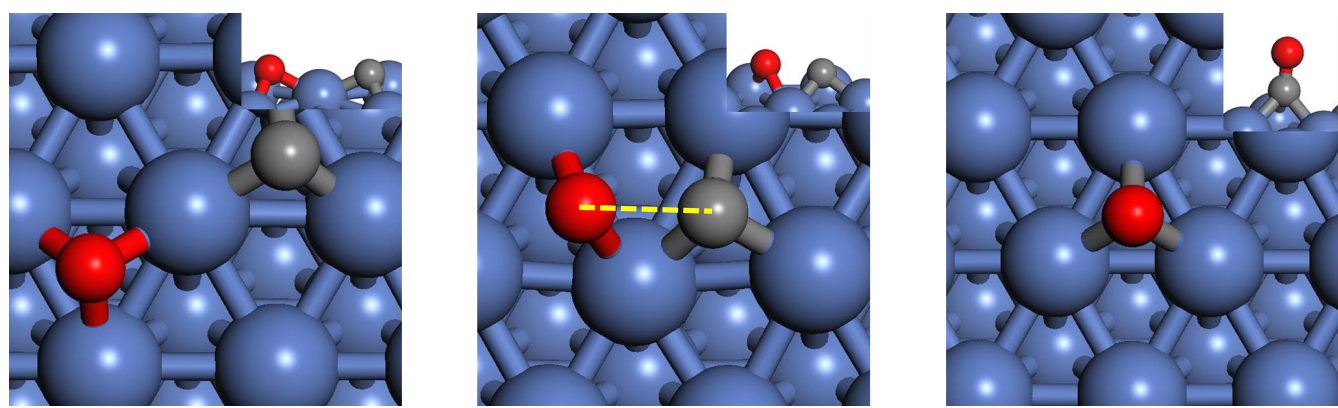

(m) $\mathrm{C}^{*}+\mathrm{O}^{*}=\mathrm{CO}^{*}\left(\mathrm{~d}_{\mathrm{C}-\mathrm{O}}=1.88 \AA\right)$
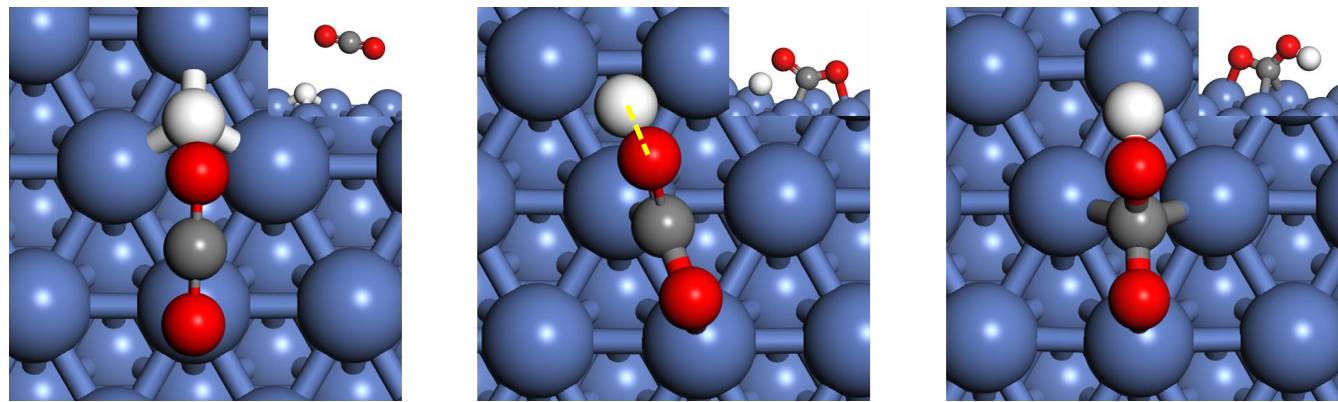

(n) $\mathrm{CO}_{2} *+\mathrm{H}^{*}=\mathrm{COOH}^{*}\left(\mathrm{~d}_{\mathrm{O}-\mathrm{H}}=1.42 \AA\right)$
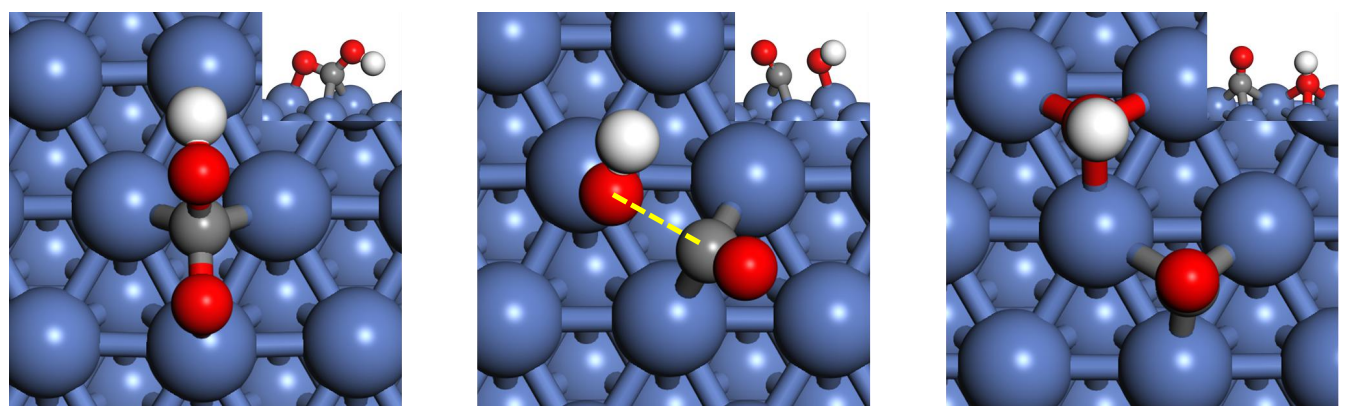

(o) $\mathrm{COOH}^{*}=\mathrm{CO}^{*}+\mathrm{OH}^{*}\left(\mathrm{~d}_{\mathrm{C}-\mathrm{O}}=1.77 \AA\right)$ 

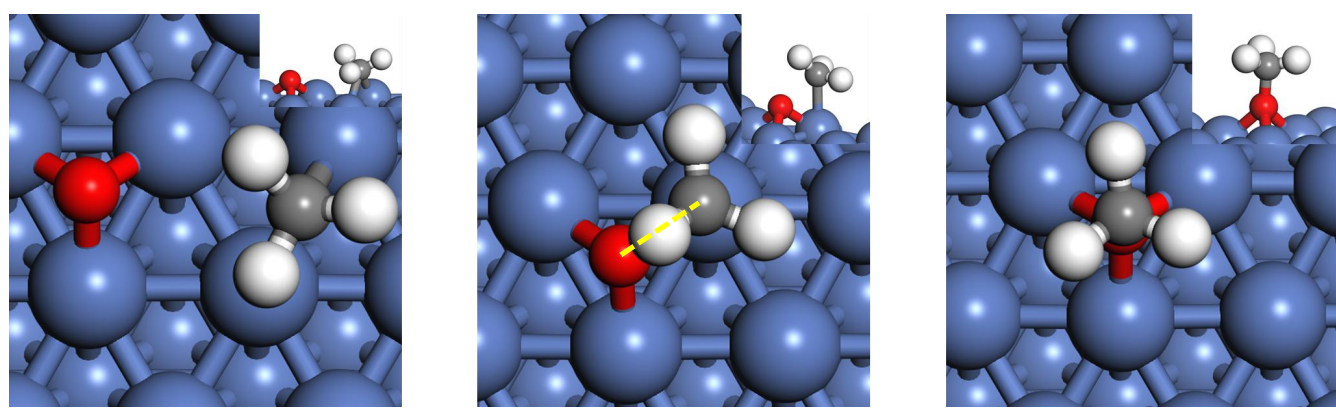

(p) $\mathrm{CH}_{3} *+\mathrm{O}^{*}=\mathrm{CH}_{3} \mathrm{O} *\left(\mathrm{~d}_{\mathrm{C}-\mathrm{O}}=1.94 \AA\right)$
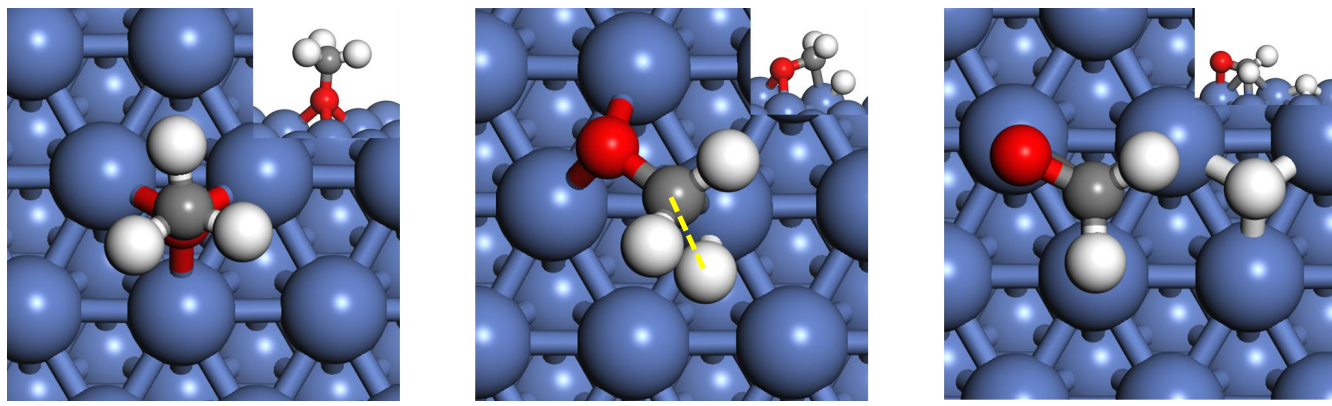

(q) $\mathrm{CH}_{3} \mathrm{O}^{*}=\mathrm{CH}_{2} \mathrm{O}^{*}+\mathrm{H}^{*}\left(\mathrm{~d}_{\mathrm{C}-\mathrm{H}}=1.53 \AA\right)$
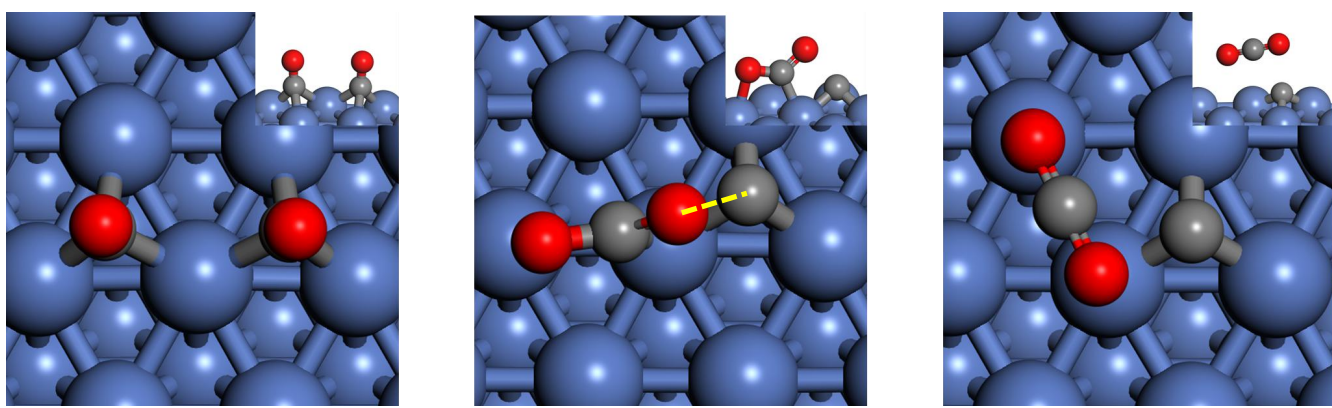

(r) $2 \mathrm{CO} *=\mathrm{CO}_{2}{ }^{*}+\mathrm{C}^{*}\left(\mathrm{~d}_{\mathrm{C}-\mathrm{O}}=\mathbf{2 . 0 0} \AA\right)$
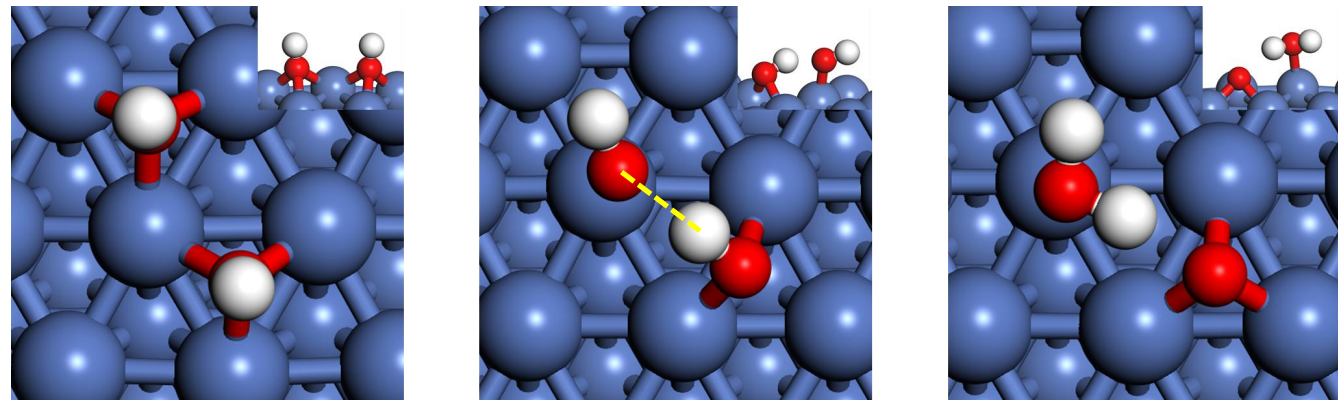

(s) $2 \mathrm{OH}^{*}=\mathrm{H}_{2} \mathrm{O} *+\mathrm{O} *\left(\mathrm{~d}_{\mathrm{O}-\mathrm{H}}=1.66 \AA\right)$

Fig. S7 The initial state, transition state and final state configurations of each elementary reaction on Ni(111). The distances between bonding and breaking atoms in the transition state structures are marked. $\mathrm{Ni}$ atoms are represented as blue balls, $\mathrm{C}$ in gray, $\mathrm{O}$ in red and $\mathrm{H}$ in white. 
Table S3 Comparison of activation energies $\left(E_{a}\right)$ for elementary reactions involved in DRM on Ni (111) with previous results. ${ }^{21}$

\begin{tabular}{|c|c|c|}
\hline Elementary reaction & $\mathrm{Ni}(111)$ & Ref. Ni(111) \\
\hline $\mathrm{CH}_{4} *=\mathrm{CH}_{3} *+\mathrm{H}^{*}$ & 0.94 & 0.91 \\
\hline $\mathrm{CH}_{3}{ }^{*}=\mathrm{CH}_{2} *+\mathrm{H}^{*}$ & 0.73 & 0.70 \\
\hline $\mathrm{CH}_{2} *=\mathrm{CH}^{*}+\mathrm{H}^{*}$ & 0.35 & 0.35 \\
\hline $\mathrm{CH}^{*}=\mathrm{C}^{*}+\mathrm{H}^{*}$ & 1.33 & 1.33 \\
\hline $\mathrm{CO}_{2} *=\mathrm{CO}^{*}+\mathrm{O}^{*}$ & 0.73 & 0.67 \\
\hline $\mathrm{CH}^{*}+\mathrm{O}^{*}=\mathrm{CHO}^{*}$ & 1.22 & 1.53 \\
\hline $\mathrm{CHO}^{*}=\mathrm{CO}^{*}+\mathrm{H}^{*}$ & 0.20 & 0.20 \\
\hline $2 \mathrm{H}^{*}=\mathrm{H}_{2} *$ & 0.99 & 0.92 \\
\hline $\mathrm{O}^{*+}+\mathrm{H}^{*}=\mathrm{OH}^{*}$ & 1.08 & 1.35 \\
\hline $\mathrm{OH}^{*+}+\mathrm{H}^{*}=\mathrm{H}_{2} \mathrm{O}^{*}$ & 1.24 & 1.33 \\
\hline $\mathrm{CH}_{2} *+\mathrm{O}^{*}=\mathrm{CH}_{2} \mathrm{O}^{*}$ & 1.11 & 1.45 \\
\hline $\mathrm{CH}_{2} \mathrm{O}^{*}=\mathrm{CHO}^{*}+\mathrm{H}^{*}$ & 0.25 & 0.36 \\
\hline $\mathrm{C}^{*}+\mathrm{O}^{*}=\mathrm{CO}^{*}$ & 1.45 & 1.59 \\
\hline $\mathrm{CO}_{2} *+\mathrm{H}^{*}=\mathrm{COOH}^{*}$ & 1.11 & 1.13 \\
\hline $\mathrm{COOH}^{*}=\mathrm{CO}^{*+}+\mathrm{OH}^{*}$ & 0.48 & 0.57 \\
\hline $\mathrm{CH}_{3} *+\mathrm{O}^{*}=\mathrm{CH}_{3} \mathrm{O}^{*}$ & 1.44 & 1.59 \\
\hline $\mathrm{CH}_{3} \mathrm{O}^{*}=\mathrm{CH}_{2} \mathrm{O}^{*}+\mathrm{H}^{*}$ & 0.94 & 0.93 \\
\hline
\end{tabular}

Note: All values are expressed in eV. Zero point energy (ZPE) and entropic contributions are not included. 


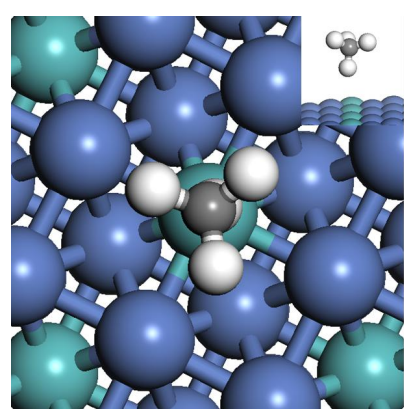

(a) $\mathrm{CH}_{4} *=\mathrm{CH}_{3} *+\mathrm{H}^{*}\left(\mathrm{~d}_{\mathrm{C}-\mathrm{H}}=1.58 \AA\right)$

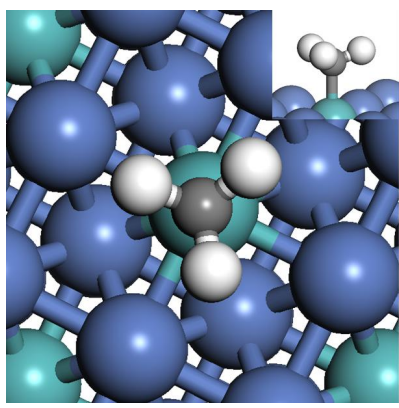

(b) $\mathrm{CH}_{3}{ }^{*}=\mathrm{CH}_{2}{ }^{*}+\mathrm{H}^{*}\left(\mathrm{~d}_{\mathrm{C}-\mathrm{H}}=1.78 \AA\right)$

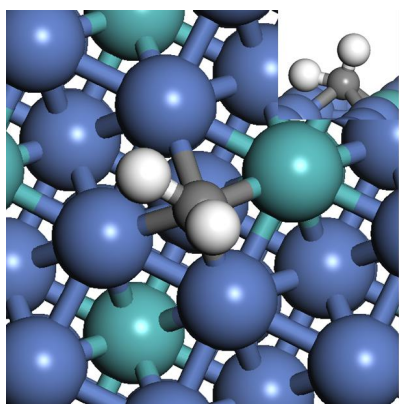

(c) $\mathrm{CH}_{2} *=\mathrm{CH}^{*}+\mathrm{H}^{*}\left(\mathrm{~d}_{\mathrm{C}-\mathrm{H}}=1.59 \AA\right)$

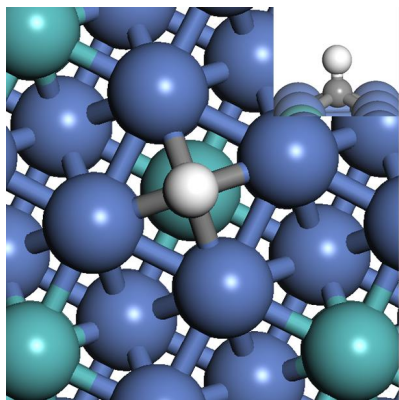

(d) $\mathrm{CH}^{*}=\mathrm{C}^{*}+\mathrm{H}^{*}\left(\mathrm{~d}_{\mathrm{C}-\mathrm{H}}=1.58 \AA\right)$

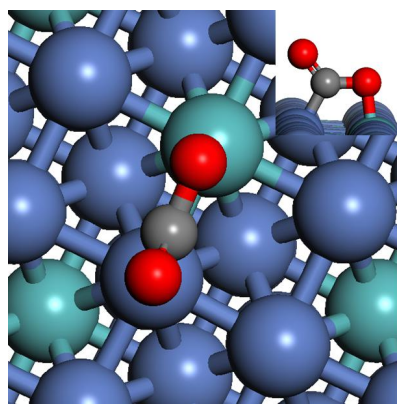

(e) $\mathrm{CO}_{2} *=\mathrm{CO}^{*}+\mathrm{O}^{*}\left(\mathrm{~d}_{\mathrm{C}-\mathrm{O}}=1.69 \AA\right)$
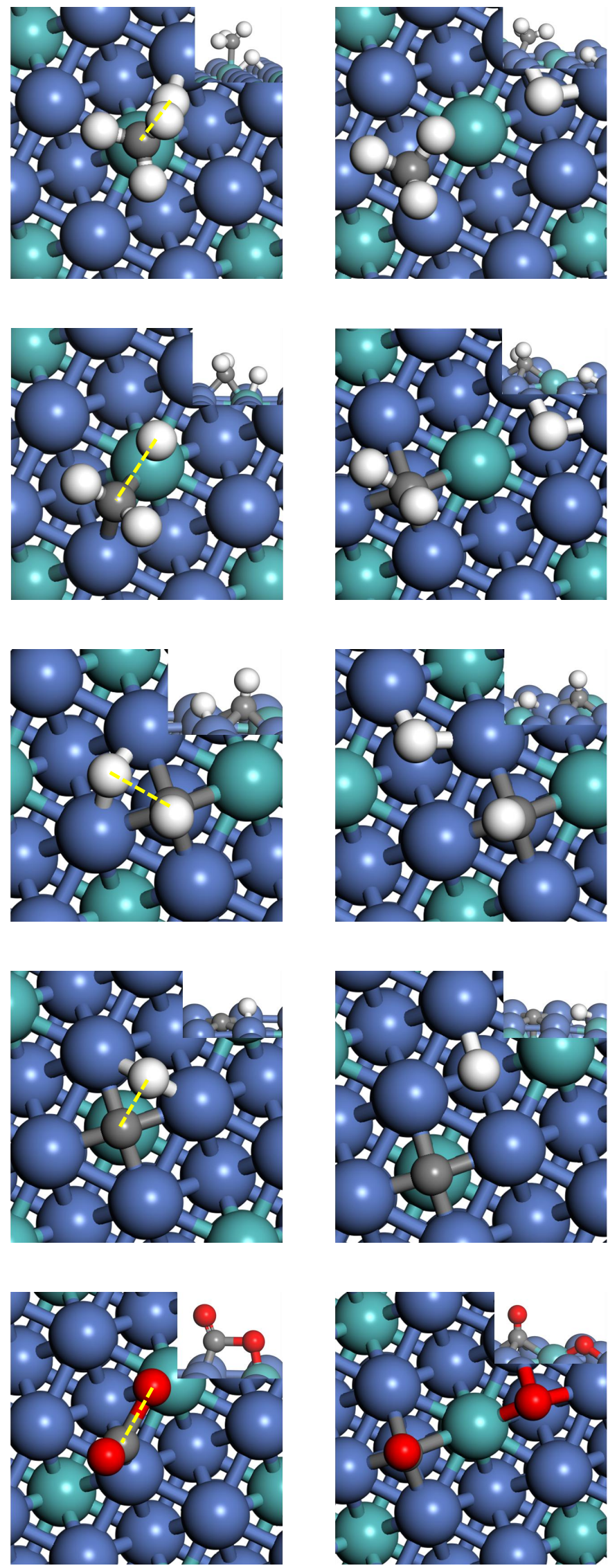


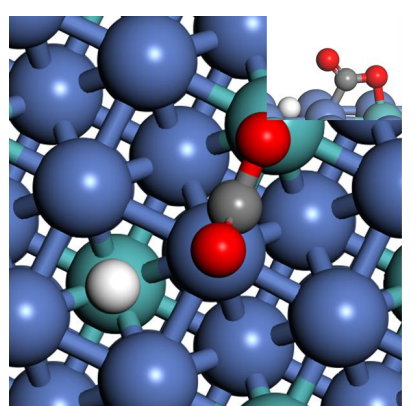

(f) $\mathrm{CO}_{2} *+\mathrm{H}^{*}=\mathrm{COOH}^{*}\left(\mathrm{~d}_{\mathrm{O}-\mathrm{H}}=1.40 \AA\right)$

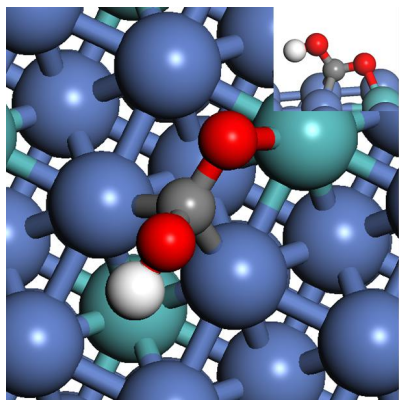

(g) $\mathrm{COOH}^{*}=\mathrm{CO}^{*}+\mathrm{OH}^{*}\left(\mathrm{~d}_{\mathrm{C}-\mathrm{O}}=1.65 \AA\right)$

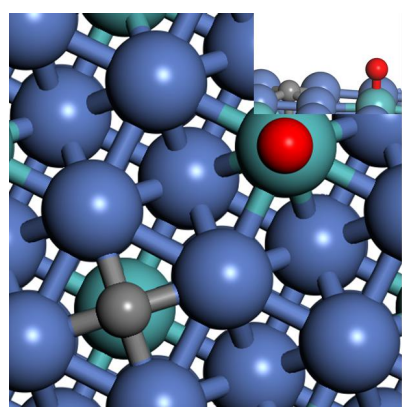

(h) $\mathrm{C}^{*}+\mathrm{O}^{*}=\mathrm{CO}^{*}\left(\mathrm{~d}_{\mathrm{C}-\mathrm{O}}=1.86 \AA\right)$

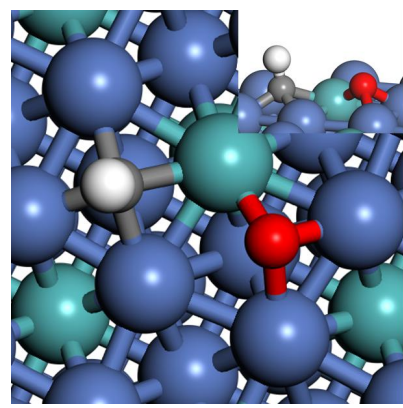

(i) $\mathrm{CH}^{*}+\mathrm{O}^{*}=\mathrm{CHO}^{*}\left(\mathrm{~d}_{\mathrm{C}-\mathrm{O}}=1.82 \AA\right)$

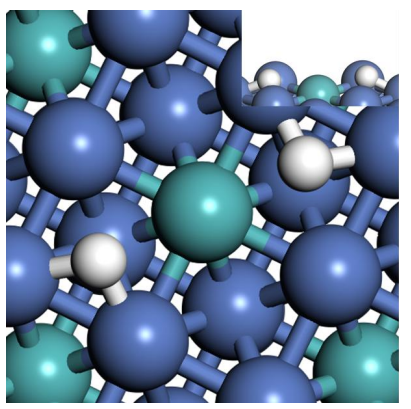

(j) $2 \mathrm{H}^{*}=\mathrm{H}_{2} *\left(\mathrm{~d}_{\mathrm{H}-\mathrm{H}}=1.13 \AA\right)$
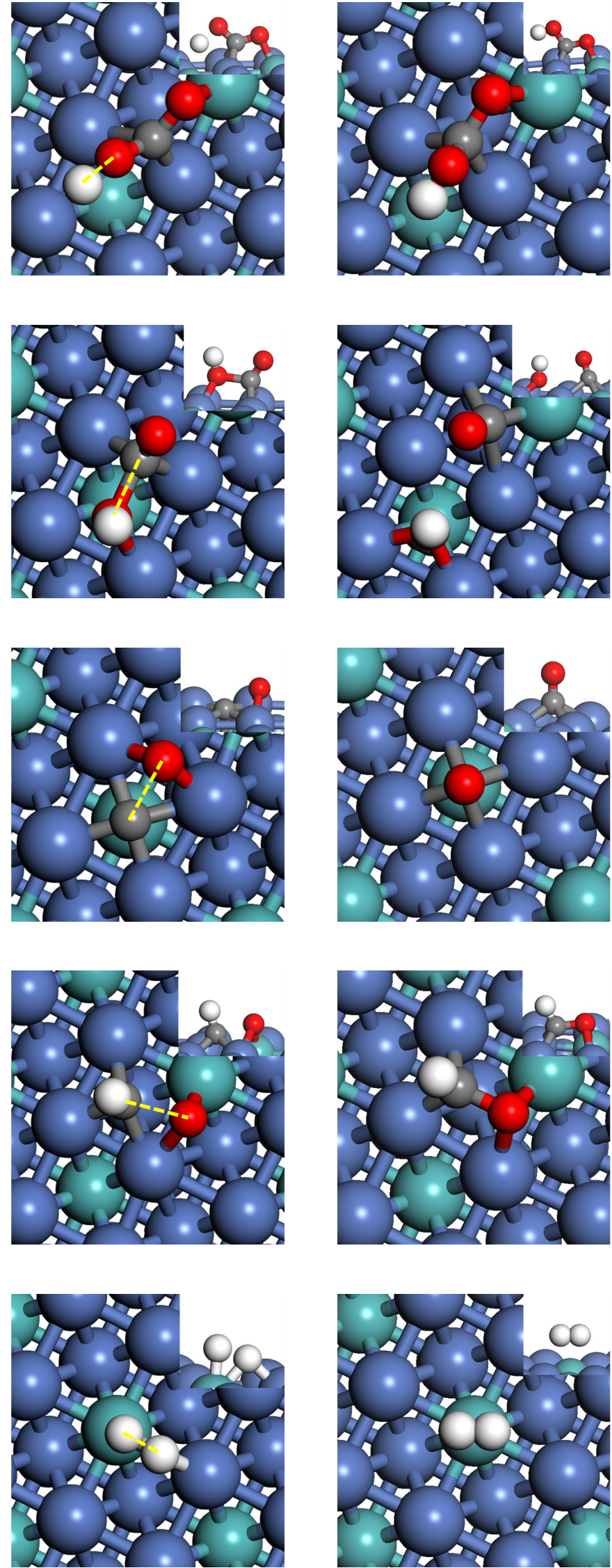


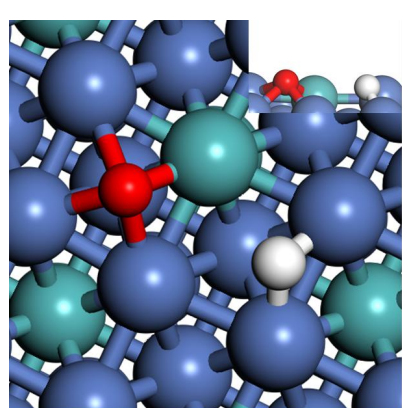

(k) $\mathrm{O}^{*}+\mathrm{H}^{*}=\mathrm{OH}^{*}\left(\mathrm{~d}_{\mathrm{O}-\mathrm{H}}=1.35 \AA\right)$

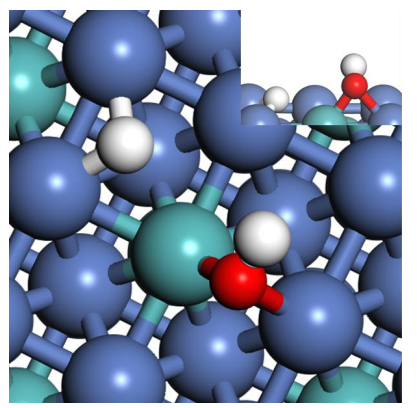

(l) $\mathrm{OH}^{*}+\mathrm{H}^{*}=\mathrm{H}_{2} \mathrm{O}^{*}\left(\mathrm{~d}_{\mathrm{O}-\mathrm{H}}=1.37 \AA\right)$

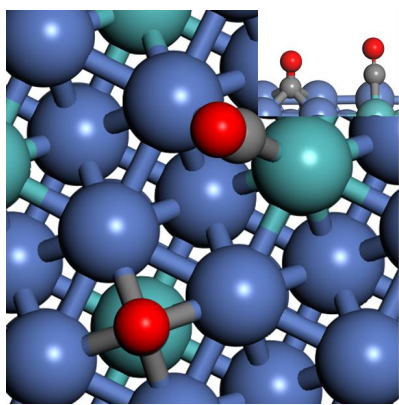

(m) $2 \mathrm{CO}^{*}=\mathrm{CO}_{2} *+\mathrm{C} *\left(\mathrm{~d}_{\mathrm{C}-\mathrm{O}}=1.68 \AA\right)$

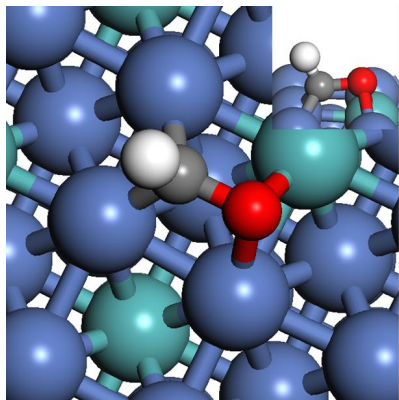

(n) $\mathrm{CHO}^{*}=\mathrm{CO}^{*}+\mathrm{H}^{*}\left(\mathrm{~d}_{\mathrm{C}-\mathrm{H}}=1.43 \AA\right)$

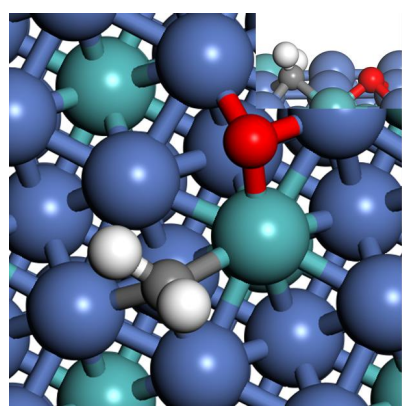

(o) $\mathrm{CH}_{2}{ }^{*}+\mathrm{O}^{*}=\mathrm{CH}_{2} \mathrm{O} *\left(\mathrm{~d}_{\mathrm{C}-\mathrm{O}}=2.00 \AA\right)$
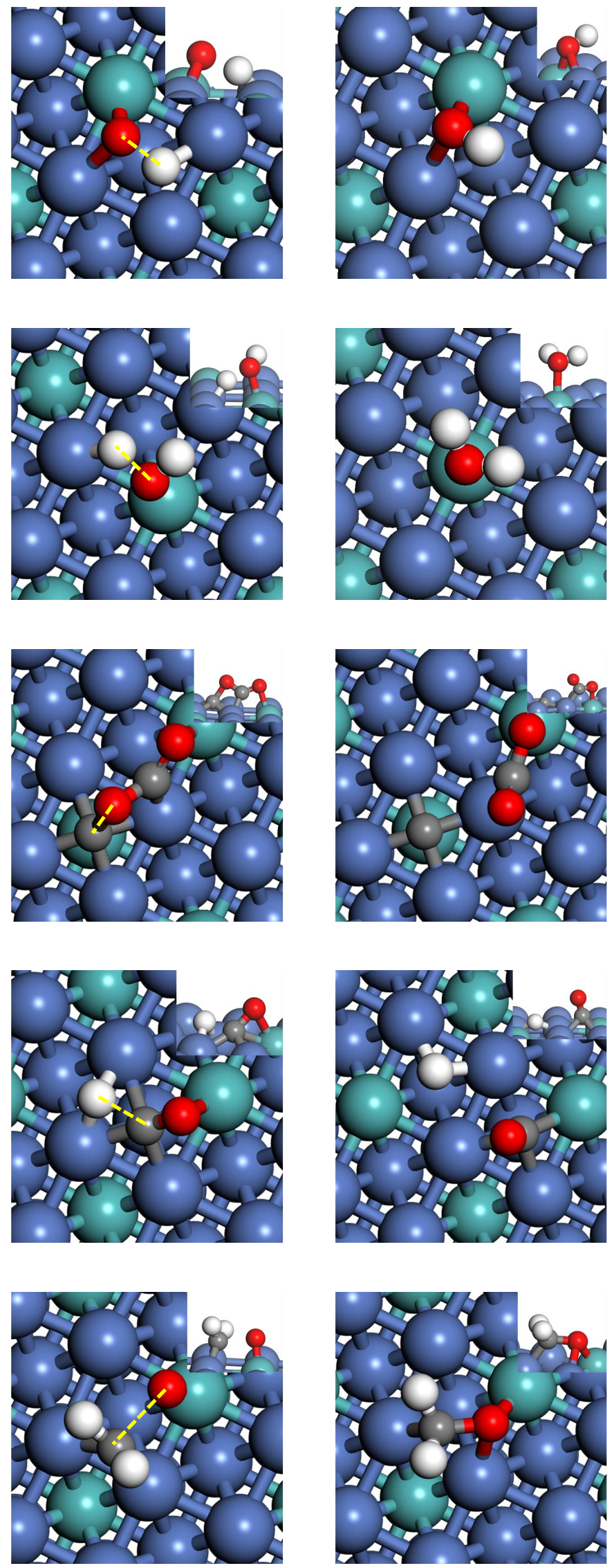

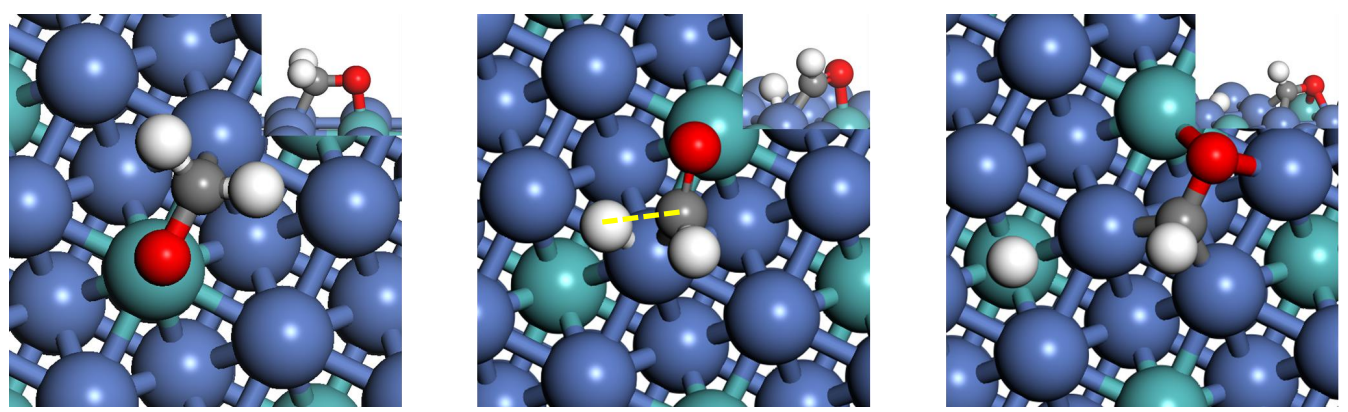

(p) $\mathrm{CH}_{2} \mathrm{O} *=\mathrm{CHO}^{*}+\mathrm{H}^{*}\left(\mathrm{~d}_{\mathrm{C}-\mathrm{H}}=1.48 \AA\right)$
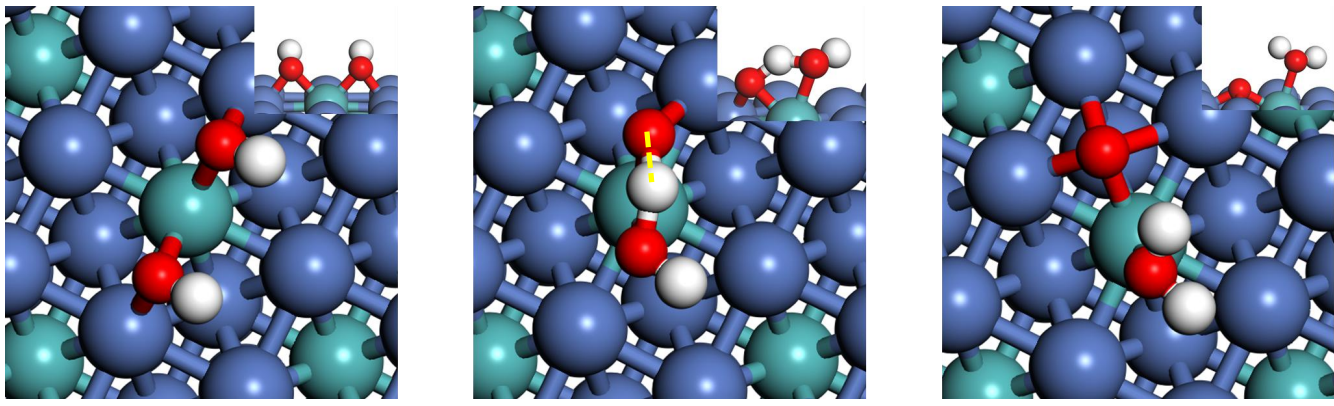

(q) $2 \mathrm{OH}^{*}=\mathrm{H}_{2} \mathrm{O} *+\mathrm{O}^{*}\left(\mathrm{~d}_{\mathrm{O}-\mathrm{H}}=1.22 \AA\right)$
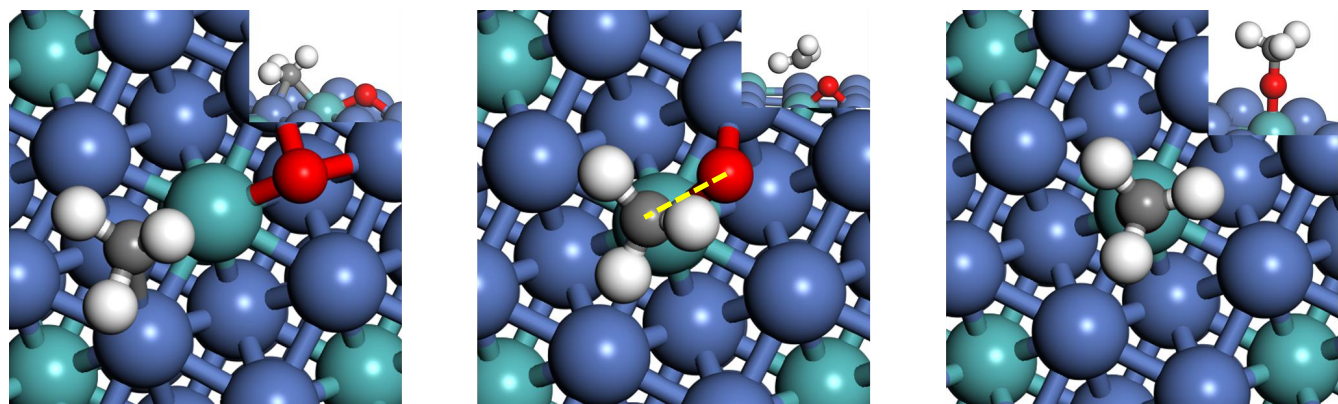

(r) $\mathrm{CH}_{3} *+\mathrm{O} *=\mathrm{CH}_{3} \mathrm{O} *\left(\mathrm{~d}_{\mathrm{C}-\mathrm{O}}=1.92 \AA\right)$
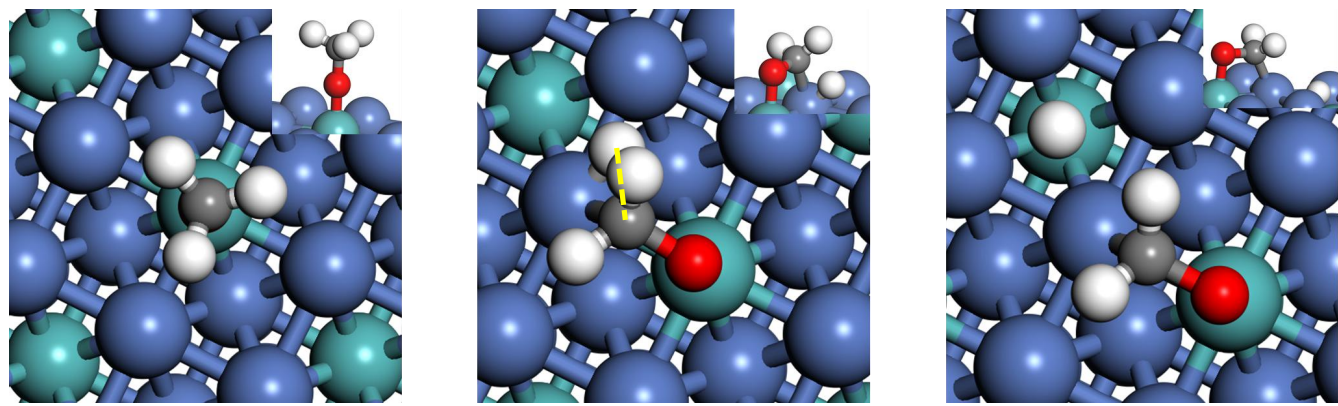

(s) $\mathrm{CH}_{3} \mathrm{O} *=\mathrm{CH}_{2} \mathrm{O}^{*}+\mathrm{H}^{*}\left(\mathrm{~d}_{\mathrm{C}-\mathrm{H}}=1.56 \AA\right)$

Fig. S8 The initial state, transition state and final state configurations of each elementary reaction on $\mathrm{MoNi}_{4}(001)$. The distances between bonding and breaking atoms in the transition state structures are marked. Ni atoms are represented as blue balls, Mo in cyan, $\mathrm{C}$ in gray, $\mathrm{O}$ in red and $\mathrm{H}$ in white. 


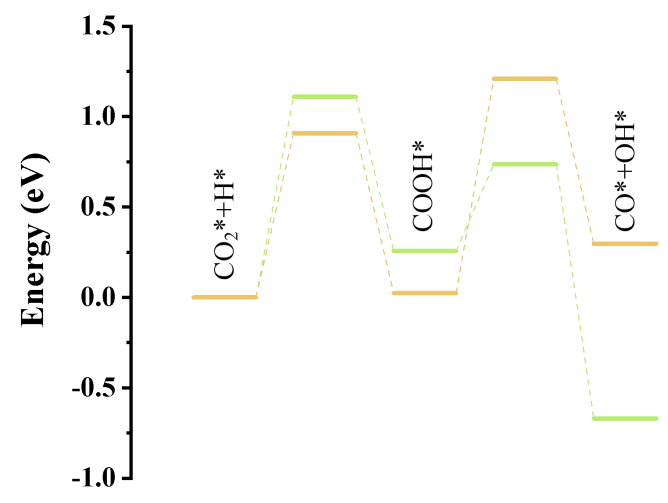

Fig. S9 Energy profiles of hydrogen-assisted $\mathrm{CO}_{2} *$ dissociation on $\mathrm{Ni}(111)$ (green) and $\mathrm{MoNi}_{4}(001)$ (orange) surfaces.

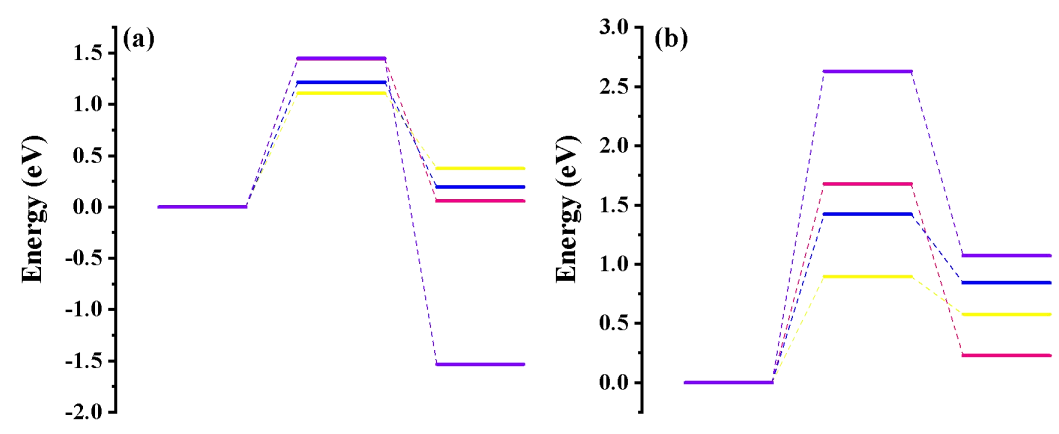

Fig. S10 Energy profiles of $\mathrm{CH}_{x}{ }^{*}(\mathrm{x}=0-3)$ oxidation on $\mathrm{Ni}(111)$ (a) and $\mathrm{MoNi}_{4}(001)$ (b) surfaces: $\mathrm{CH}_{3}-\mathrm{O}$ oxidation (red), $\mathrm{CH}_{2}-\mathrm{O}$ oxidation (yellow), $\mathrm{CH}-\mathrm{O}$ oxidation (blue), and $\mathrm{C}-\mathrm{O}$ oxidation (purple).

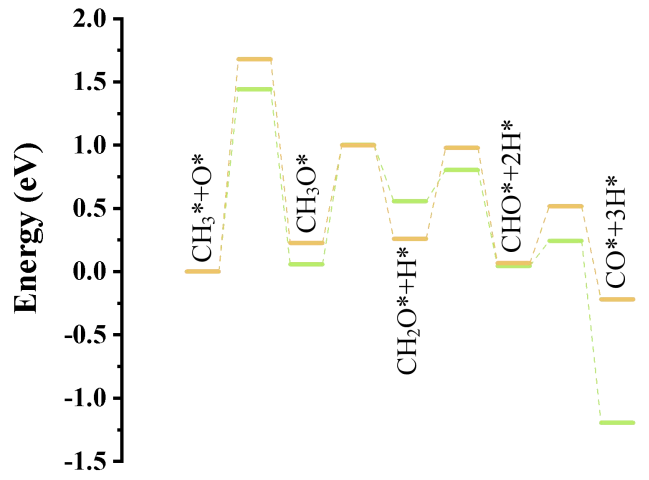

Fig. S11 Energy profiles of $\mathrm{CH}_{3}-\mathrm{O}$ oxidation and subsequent dissociation on $\mathrm{Ni}(111)$ (green) and $\mathrm{MoNi}_{4}(001)$ (orange) surfaces. 


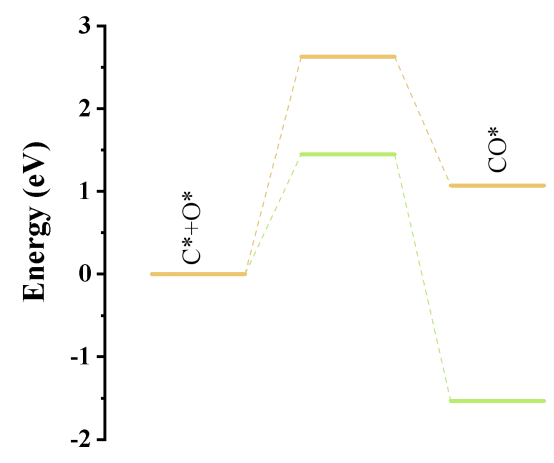

Fig. S12 Energy profiles of C-O oxidation on $\mathrm{Ni}(111)$ (green) and $\mathrm{MoNi}_{4}(001)$ (orange) surfaces.

Table S4 Activation free energies for all the forward $\left(G_{a}\right)$ and reverse $\left(G_{a}^{-1}\right)$ elementary reactions involved in DRM on $\mathrm{Ni}(111)$ and $\mathrm{MoNi}_{4}(001)$ surfaces.

\begin{tabular}{|c|c|c|c|c|}
\hline \multirow{2}{*}{ Reactions } & \multicolumn{2}{|c|}{$\mathrm{Ni}(111)$} & \multicolumn{2}{|c|}{$\mathrm{MoNi}_{4}(001)$} \\
\hline & $G_{a}(\mathrm{eV})$ & $G_{a}^{-1}(\mathrm{eV})$ & $G_{a}(\mathrm{eV})$ & $G_{a}^{-1}(\mathrm{eV})$ \\
\hline $\mathrm{CH}_{4}(\mathrm{~g})=\mathrm{CH}_{3} *+\mathrm{H}^{*}$ & 2.53 & 0.88 & 2.10 & 0.76 \\
\hline $\mathrm{CH}_{3} *=\mathrm{CH}_{2} *+\mathrm{H}^{*}$ & 0.64 & 0.61 & 0.75 & 1.12 \\
\hline $\mathrm{CH}_{2} *=\mathrm{CH}^{*}+\mathrm{H}^{*}$ & 0.35 & 0.66 & 0.21 & 0.72 \\
\hline $\mathrm{CH}^{*}=\mathrm{C}^{*}+\mathrm{H}^{*}$ & 1.25 & 0.66 & 0.68 & 0.72 \\
\hline $\mathrm{CO}_{2}(\mathrm{~g})=\mathrm{CO}^{*}+\mathrm{O}^{*}$ & 2.08 & 1.61 & 0.94 & 1.39 \\
\hline $\mathrm{CO}_{2}(\mathrm{~g})+\mathrm{H}^{*}=\mathrm{COOH}^{*}$ & 2.40 & 0.72 & 1.59 & 0.75 \\
\hline $\mathrm{COOH}^{*}=\mathrm{CO}^{*}+\mathrm{OH}^{*}$ & 0.51 & 1.47 & 1.19 & 1.02 \\
\hline $\mathrm{CH}_{3} *+\mathrm{O}^{*}=\mathrm{CH}_{3} \mathrm{O} *$ & 1.53 & 1.38 & 1.80 & 1.44 \\
\hline $\mathrm{CH}_{3} \mathrm{O}^{*}=\mathrm{CH}_{2} \mathrm{O}^{*+}+\mathrm{H}^{*}$ & 0.87 & 0.57 & 0.73 & 0.85 \\
\hline $\mathrm{CH}_{2} *+\mathrm{O}^{*}=\mathrm{CH}_{2} \mathrm{O}^{*}$ & 1.22 & 0.83 & 1.00 & 0.34 \\
\hline $\mathrm{CH}_{2} \mathrm{O}^{*}=\mathrm{CHO}^{*}+\mathrm{H}^{*}$ & 0.27 & 0.82 & 0.65 & 1.01 \\
\hline $\mathrm{CH}^{*}+\mathrm{O}^{*}=\mathrm{CHO}^{*}$ & 1.26 & 1.09 & 1.51 & 0.62 \\
\hline $\mathrm{CHO}^{*}=\mathrm{CO}^{*}+\mathrm{H}^{*}$ & 0.22 & 1.55 & 0.39 & 0.84 \\
\hline $\mathrm{C}^{*}+\mathrm{O}^{*}=\mathrm{CO}^{*}$ & 1.48 & 3.04 & 2.71 & 1.64 \\
\hline $2 \mathrm{H}^{*}=\mathrm{H}_{2}(\mathrm{~g})$ & 0.92 & 1.02 & 0.75 & 0.71 \\
\hline $\mathrm{O}^{*+}+\mathrm{H}^{*}=\mathrm{OH}^{*}$ & 1.06 & 0.89 & 0.91 & 0.52 \\
\hline $\mathrm{OH}^{*}+\mathrm{H}^{*}=\mathrm{H}_{2} \mathrm{O}(\mathrm{g})$ & 1.20 & 2.13 & 1.16 & 1.43 \\
\hline $2 \mathrm{CO}^{*}=\mathrm{CO}_{2}(\mathrm{~g})+\mathrm{C}^{*}$ & 3.38 & 2.51 & 1.60 & 2.05 \\
\hline $2 \mathrm{OH}^{*}=\mathrm{H}_{2} \mathrm{O}(\mathrm{g})+\mathrm{O}^{*}$ & 0.30 & 1.95 & 0.69 & 1.74 \\
\hline $\mathrm{CO}^{*}=\mathrm{CO}(\mathrm{g})$ & 0.12 & 0.00 & 0.16 & 0.00 \\
\hline
\end{tabular}

Note: Zero point energy (ZPE) and entropic contributions are included. 


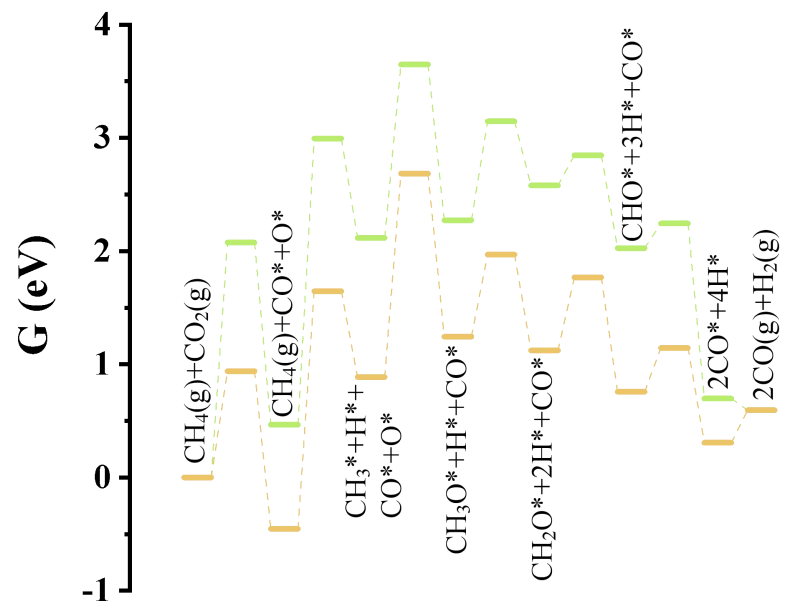

Fig. S13 Free energy profiles of DRM reaction through $\mathrm{CH}_{3}-\mathrm{O}$ oxidation path on $\mathrm{Ni}(111)$ (green) and $\mathrm{MoNi}_{4}(001)$ (orange) surfaces at $1073.15 \mathrm{~K}$.

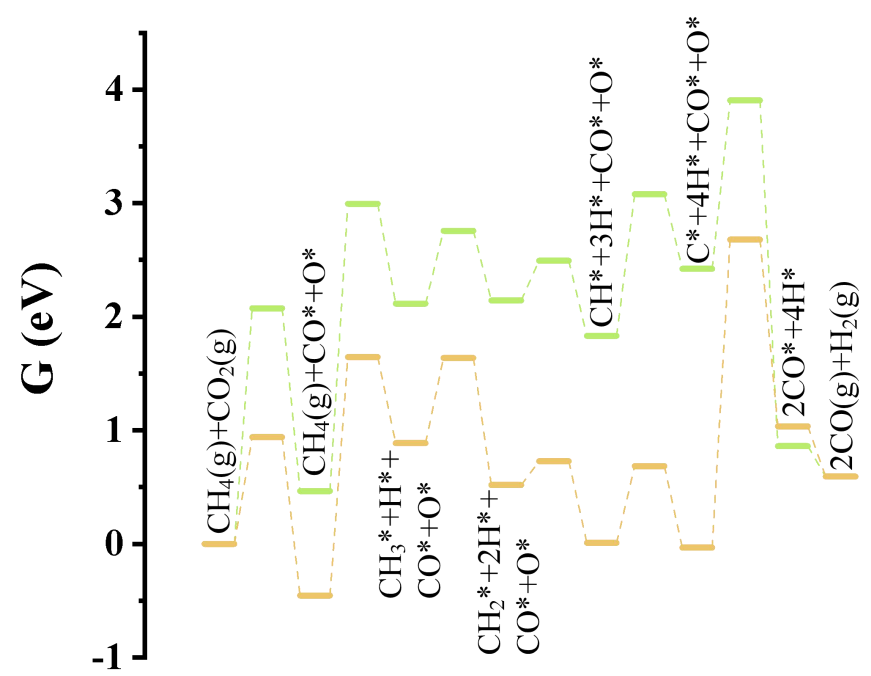

Fig. S14 Free energy profiles of DRM reaction through C-O oxidation path on Ni(111) (green) and $\mathrm{MoNi}_{4}(001)$ (orange) surfaces at $1073.15 \mathrm{~K}$. 


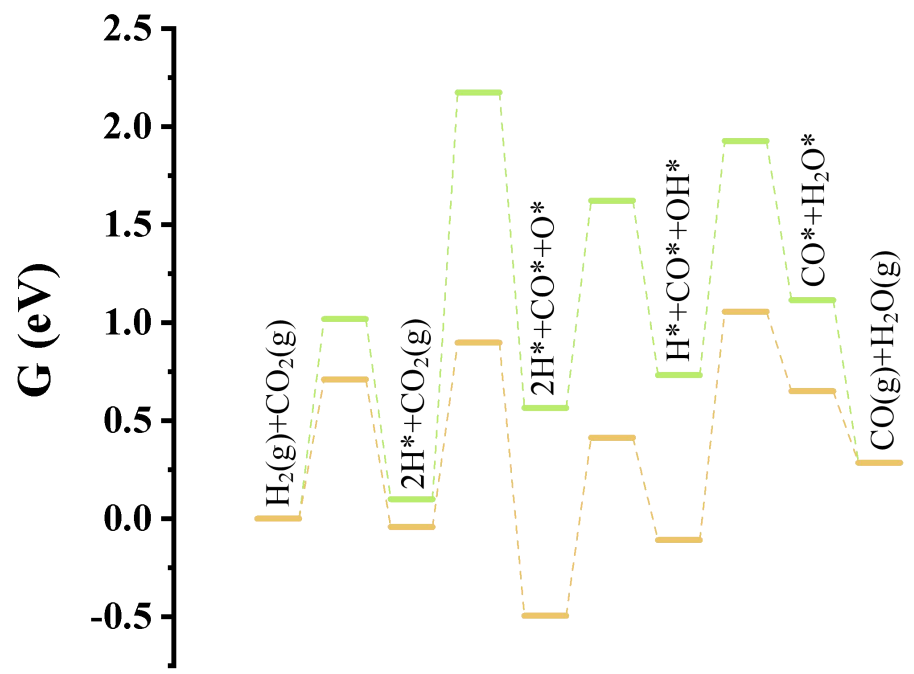

Fig. S15 Free energy profiles of RWGS reaction on $\mathrm{Ni}(111)$ (green) and $\mathrm{MoNi}_{4}(001)$ (orange) surfaces at 1073.15 K.

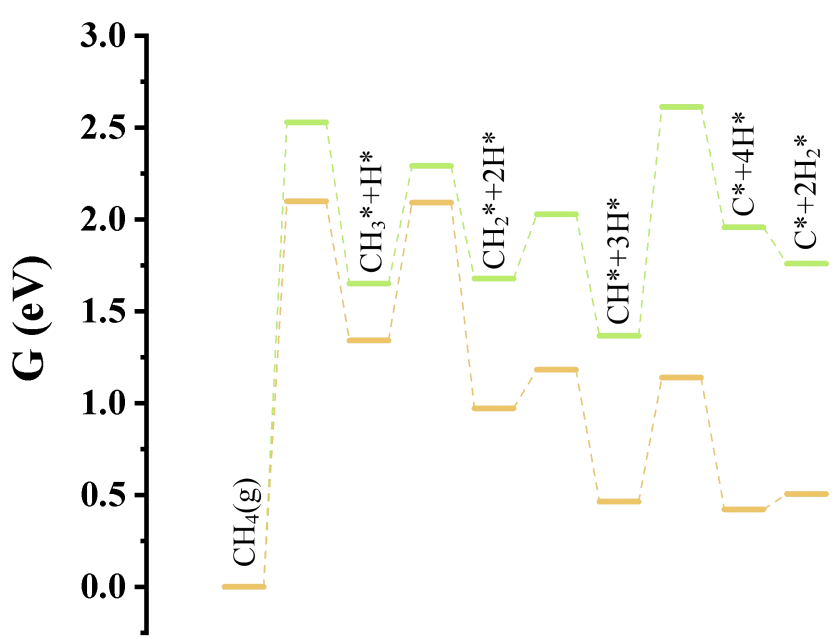

Fig. S16 Free energy profiles of $\mathrm{CH}_{4}$ cracking reaction on $\mathrm{Ni}(111)$ (green) and $\mathrm{MoNi}_{4}(001)$ (orange) surfaces at $1073.15 \mathrm{~K}$. 


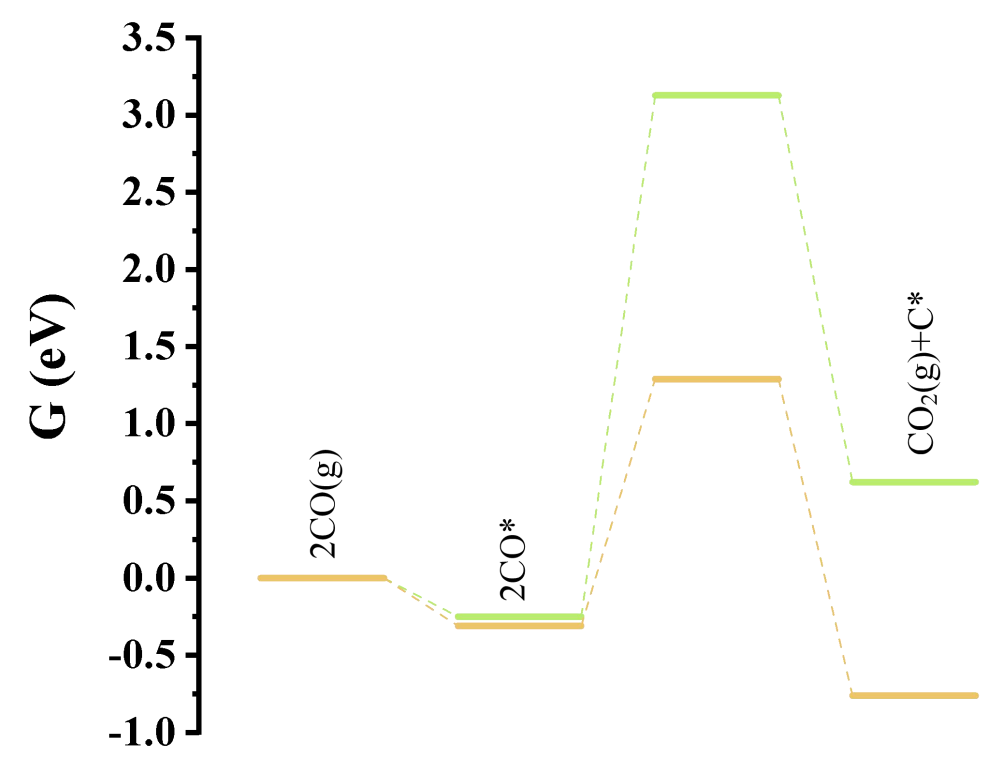

Fig. S17 Free energy profiles of the Boudouard reaction on $\mathrm{Ni}(111)$ (green) and $\mathrm{MoNi}_{4}(001)$ (orange) surfaces at $1073.15 \mathrm{~K}$.

Table S5 Energy barrier $\left(E_{a}\right)$ and reaction energies $(\Delta E)$ of $\mathrm{C}^{*}$ diffusion process on $\mathrm{Ni}(111)$ and $\mathrm{MoNi}_{4}(001)$.

\begin{tabular}{ccc}
\hline C-diffusion & $\mathrm{Ni}(111)$ & $\mathrm{MoNi}_{4}(001)$ \\
\hline$E_{a}(\mathrm{eV})$ & 0.34 & 2.28 \\
$\Delta E(\mathrm{eV})$ & 0.06 & 0.07 \\
\hline
\end{tabular}

Note: Zero point energy (ZPE) and entropic contributions are not included.
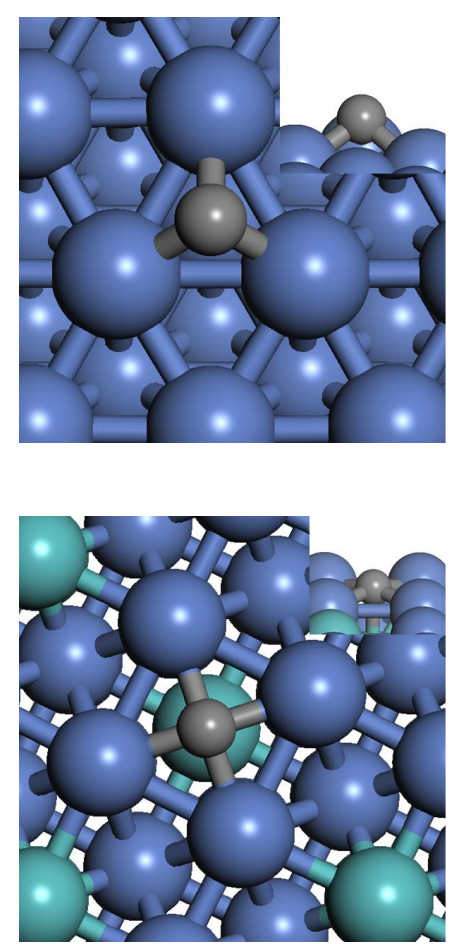

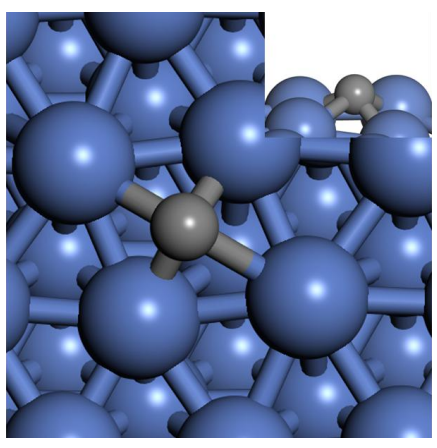

(a)

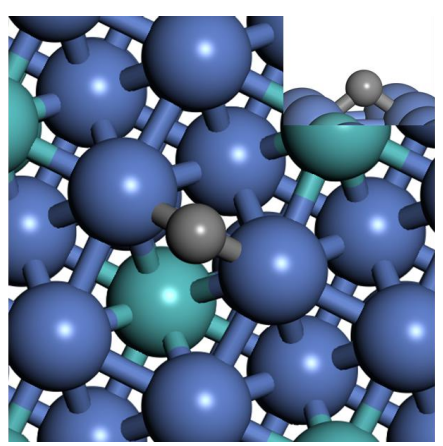

(b)
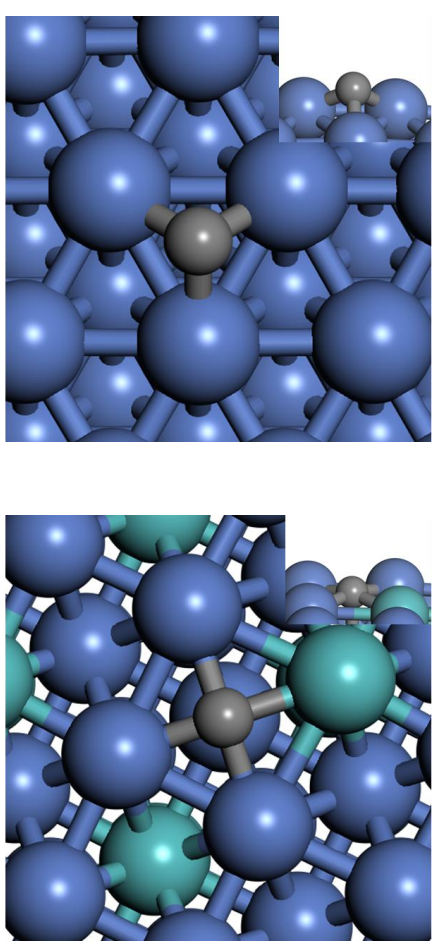

Fig. S18 The initial state, transition state and final state configurations of $C^{*}$ diffusion process on $\mathrm{Ni}(111)$ (a) and $\mathrm{MoNi}_{4}(001)$ (b) surfaces. 
Table S6 Activation free energies and pre-exponential factors for all the forward $\left(G_{a}, A\right)$ and reverse $\left(G_{a}^{-1}, A^{-1}\right)$ elementary reactions involved in DRM on $\mathrm{Ni}(111)$ in the microkinetic model at $1073.15 \mathrm{~K}$ and 1 bar.

\begin{tabular}{|c|c|c|c|c|c|}
\hline Label & Elementary reaction & $G_{a}(\mathrm{eV})$ & $A\left(\mathrm{~s}^{-1}\right)$ & $G_{a}^{-1}(\mathrm{eV})$ & $A^{-1}\left(\mathrm{~s}^{-1}\right)$ \\
\hline $\mathrm{R} 1$ & $\mathrm{CH}_{4} *+*=\mathrm{CH}_{3} *+\mathrm{H}^{*}$ & 0.93 & $2.24 \mathrm{E}+13$ & 0.88 & $2.24 \mathrm{E}+13$ \\
\hline $\mathrm{R} 2$ & $\mathrm{CH}_{3} *+*=\mathrm{CH}_{2} *+\mathrm{H}^{*}$ & 0.64 & $2.24 \mathrm{E}+13$ & 0.61 & $2.24 \mathrm{E}+13$ \\
\hline $\mathrm{R} 3$ & $\mathrm{CH}_{2}{ }^{*+*}=\mathrm{CH}^{*+} \mathrm{H}^{*}$ & 0.35 & $2.24 \mathrm{E}+13$ & 0.66 & $2.24 \mathrm{E}+13$ \\
\hline R4 & $\mathrm{CH}^{*+*}=\mathrm{C}^{*+\mathrm{H}^{*}}$ & 1.25 & $2.24 \mathrm{E}+13$ & 0.66 & $2.24 \mathrm{E}+13$ \\
\hline R5 & $\mathrm{CO}_{2} *+*=\mathrm{CO} *+\mathrm{O}^{*}$ & 0.78 & $2.24 \mathrm{E}+13$ & 1.61 & $2.24 \mathrm{E}+13$ \\
\hline R6 & $\mathrm{CO}_{2} *+\mathrm{H}^{*}=\mathrm{COOH}^{*+*}$ & 1.11 & $2.24 \mathrm{E}+13$ & 0.72 & $2.24 \mathrm{E}+13$ \\
\hline R7 & $\mathrm{COOH}^{*+*}=\mathrm{CO}^{*}+\mathrm{OH}^{*}$ & 0.51 & $2.24 \mathrm{E}+13$ & 1.47 & $2.24 \mathrm{E}+13$ \\
\hline $\mathrm{R} 8$ & $\mathrm{CH}_{3} *+\mathrm{O}^{*}=\mathrm{CH}_{3} \mathrm{O}^{*+*}$ & 1.53 & $2.24 \mathrm{E}+13$ & 1.38 & $2.24 \mathrm{E}+13$ \\
\hline R9 & $\mathrm{CH}_{3} \mathrm{O}^{*+*}=\mathrm{CH}_{2} \mathrm{O}^{*+} \mathrm{H}^{*}$ & 0.87 & $2.24 \mathrm{E}+13$ & 0.57 & $2.24 \mathrm{E}+13$ \\
\hline $\mathrm{R} 10$ & $\mathrm{CH}_{2}{ }^{*}+\mathrm{O}^{*}=\mathrm{CH}_{2} \mathrm{O}^{*+*}$ & 1.22 & $2.24 \mathrm{E}+13$ & 0.83 & $2.24 \mathrm{E}+13$ \\
\hline R11 & $\mathrm{CH}_{2} \mathrm{O}^{*+*}=\mathrm{CHO}^{*+}+\mathrm{H}^{*}$ & 0.27 & $2.24 \mathrm{E}+13$ & 0.82 & $2.24 \mathrm{E}+13$ \\
\hline $\mathrm{R} 12$ & 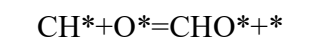 & 1.26 & $2.24 \mathrm{E}+13$ & 1.09 & $2.24 \mathrm{E}+13$ \\
\hline $\mathrm{R} 13$ & $\mathrm{CHO}^{*+*}=\mathrm{CO}^{*}+\mathrm{H}^{*}$ & 0.22 & $2.24 \mathrm{E}+13$ & 1.55 & $2.24 \mathrm{E}+13$ \\
\hline $\mathrm{R} 14$ & $\mathrm{C}^{*+} \mathrm{O}^{*}=\mathrm{CO}^{*+*}$ & 1.48 & $2.24 \mathrm{E}+13$ & 3.04 & $2.24 \mathrm{E}+13$ \\
\hline $\mathrm{R} 15$ & $2 \mathrm{H}^{*}=\mathrm{H}_{2}(\mathrm{~g})+2 *$ & 0.92 & $2.24 \mathrm{E}+13$ & 1.02 & $2.24 \mathrm{E}+13$ \\
\hline $\mathrm{R} 16$ & $\mathrm{O}^{*+}+\mathrm{H}^{*}=\mathrm{OH}^{*+*}$ & 1.06 & $2.24 \mathrm{E}+13$ & 0.89 & $2.24 \mathrm{E}+13$ \\
\hline $\mathrm{R} 17$ & $\mathrm{OH}^{*+}+\mathrm{H}^{*}=\mathrm{H}_{2} \mathrm{O}^{*+*}$ & 1.20 & $2.24 \mathrm{E}+13$ & 0.81 & $2.24 \mathrm{E}+13$ \\
\hline $\mathrm{R} 18$ & $2 \mathrm{CO}^{*}=\mathrm{CO}_{2} *+\mathrm{C}^{*}$ & 3.38 & $2.24 \mathrm{E}+13$ & 1.21 & $2.24 \mathrm{E}+13$ \\
\hline R19 & $2 \mathrm{OH}^{*}=\mathrm{H}_{2} \mathrm{O} *+\mathrm{O}^{*}$ & 0.30 & $2.24 \mathrm{E}+13$ & 0.63 & $2.24 \mathrm{E}+13$ \\
\hline M1 & $\mathrm{CO}^{*}=\mathrm{CO}(\mathrm{g})^{+*}$ & 1.45 & $5.27 \mathrm{E}+18$ & 0.00 & $9.43 \mathrm{E}+02$ \\
\hline M2 & $\mathrm{CO}_{2}(\mathrm{~g})^{+*}=\mathrm{CO}_{2} *$ & 0.00 & $7.52 \mathrm{E}+02$ & 0.28 & $2.05 \mathrm{E}+19$ \\
\hline M3 & $\mathrm{H}_{2} \mathrm{O}^{*}=\mathrm{H}_{2} \mathrm{O}(\mathrm{g})^{+*}$ & 0.48 & $1.17 \mathrm{E}+17$ & 0.00 & $1.18 \mathrm{E}+03$ \\
\hline M4 & $\mathrm{CH}_{4}(\mathrm{~g})+*=\mathrm{CH}_{4} *$ & 0.00 & $1.25 \mathrm{E}+03$ & 0.24 & $9.18 \mathrm{E}+16$ \\
\hline
\end{tabular}

Note: In the table, M1-M4 show adsorption energy instead of adsorption free energy. On Ni(111), the adsorption of $\mathrm{CO}_{2}$ and $\mathrm{CH}_{4}$ is too weak to make the simulation difficult to carry out. We have enlarged the adsorption energy of $\mathrm{CO}_{2}$ and $\mathrm{CH}_{4}$ in the same proportion (20\%) during the simulation. Since the adsorption process is generally not considered to be a key step in determining the reaction rate, it is understandable that we operate in this way. 
Table S7 Activation free energies and pre-exponential factors for all the forward $\left(G_{a}, A\right)$ and reverse $\left(G_{a}^{-1}, A^{-1}\right)$ elementary reactions involved in $\mathrm{DRM}$ on $\mathrm{MoNi}_{4}(001)$ in the microkinetic model at $1073.15 \mathrm{~K}$ and 1 bar.

\begin{tabular}{|c|c|c|c|c|c|}
\hline Label & Elementary reaction & $G_{a}(\mathrm{eV})$ & $A\left(\mathrm{~s}^{-1}\right)$ & $G_{a}^{-1}(\mathrm{eV})$ & $A^{-1}\left(\mathrm{~s}^{-1}\right)$ \\
\hline $\mathrm{R} 1$ & $\mathrm{CH}_{4} *+*=\mathrm{CH}_{3} *+\mathrm{H}^{*}$ & 0.48 & $2.24 \mathrm{E}+13$ & 0.76 & $2.24 \mathrm{E}+13$ \\
\hline $\mathrm{R} 2$ & $\mathrm{CH}_{3} *+*=\mathrm{CH}_{2} *+\mathrm{H}^{*}$ & 0.75 & $2.24 \mathrm{E}+13$ & 1.12 & $2.24 \mathrm{E}+13$ \\
\hline $\mathrm{R} 3$ & $\mathrm{CH}_{2} *+*=\mathrm{CH}^{*+} \mathrm{H}^{*}$ & 0.21 & $2.24 \mathrm{E}+13$ & 0.72 & $2.24 \mathrm{E}+13$ \\
\hline $\mathrm{R} 4$ & $\mathrm{CH}^{*+*}=\mathrm{C}^{*+} \mathrm{H}^{*}$ & 0.68 & $2.24 \mathrm{E}+13$ & 0.72 & $2.24 \mathrm{E}+13$ \\
\hline R5 & $\mathrm{CO}_{2} *+*=\mathrm{CO}^{*+}+\mathrm{O}^{*}$ & 0.30 & $2.24 \mathrm{E}+13$ & 1.39 & $2.24 \mathrm{E}+13$ \\
\hline R6 & $\mathrm{CO}_{2} *+\mathrm{H}^{*}=\mathrm{COOH}^{*+*}$ & 0.95 & $2.24 \mathrm{E}+13$ & 0.75 & $2.24 \mathrm{E}+13$ \\
\hline R7 & $\mathrm{COOH}^{*+*}=\mathrm{CO}^{*+}+\mathrm{OH}^{*}$ & 1.19 & $2.24 \mathrm{E}+13$ & 1.02 & $2.24 \mathrm{E}+13$ \\
\hline $\mathrm{R} 8$ & $\mathrm{CH}_{3} *+\mathrm{O}^{*}=\mathrm{CH}_{3} \mathrm{O}^{*+} *$ & 1.80 & $2.24 \mathrm{E}+13$ & 1.44 & $2.24 \mathrm{E}+13$ \\
\hline R9 & $\mathrm{CH}_{3} \mathrm{O}^{*+*}=\mathrm{CH}_{2} \mathrm{O}^{*}+\mathrm{H}^{*}$ & 0.73 & $2.24 \mathrm{E}+13$ & 0.85 & $2.24 \mathrm{E}+13$ \\
\hline $\mathrm{R} 10$ & $\mathrm{CH}_{2}{ }^{*}+\mathrm{O}^{*}=\mathrm{CH}_{2} \mathrm{O}^{*+*}$ & 1.00 & $2.24 \mathrm{E}+13$ & 0.34 & $2.24 \mathrm{E}+13$ \\
\hline R11 & $\mathrm{CH}_{2} \mathrm{O}^{*+*}=\mathrm{CHO}^{*}+\mathrm{H}^{*}$ & 0.65 & $2.24 \mathrm{E}+13$ & 1.01 & $2.24 \mathrm{E}+13$ \\
\hline $\mathrm{R} 12$ & $\mathrm{CH}^{*+}+\mathrm{O}^{*}=\mathrm{CHO}^{*+*}$ & 1.51 & $2.24 \mathrm{E}+13$ & 0.62 & $2.24 \mathrm{E}+13$ \\
\hline $\mathrm{R} 13$ & $\mathrm{CHO}^{*+*}=\mathrm{CO}^{*+}+\mathrm{H}^{*}$ & 0.39 & $2.24 \mathrm{E}+13$ & 0.84 & $2.24 \mathrm{E}+13$ \\
\hline $\mathrm{R} 14$ & $\mathrm{C}^{*+} \mathrm{O}^{*}=\mathrm{CO}^{*+*}$ & 2.71 & $2.24 \mathrm{E}+13$ & 1.64 & $2.24 \mathrm{E}+13$ \\
\hline $\mathrm{R} 15$ & $2 \mathrm{H}^{*}=\mathrm{H}_{2}(\mathrm{~g})+2^{*}$ & 0.75 & $2.24 \mathrm{E}+13$ & 0.07 & $2.24 \mathrm{E}+13$ \\
\hline $\mathrm{R} 16$ & $\mathrm{O}^{*+}+\mathrm{H}^{*}=\mathrm{OH}^{*+*}$ & 0.91 & $2.24 \mathrm{E}+13$ & 0.52 & $2.24 \mathrm{E}+13$ \\
\hline $\mathrm{R} 17$ & $\mathrm{OH}^{*+}+\mathrm{H}^{*}=\mathrm{H}_{2} \mathrm{O}^{*+*}$ & 1.16 & $2.24 \mathrm{E}+13$ & 0.41 & $2.24 \mathrm{E}+13$ \\
\hline $\mathrm{R} 18$ & $2 \mathrm{CO}^{*}=\mathrm{CO}_{2}{ }^{*}+\mathrm{C}^{*}$ & 1.60 & $2.24 \mathrm{E}+13$ & 1.41 & $2.24 \mathrm{E}+13$ \\
\hline $\mathrm{R} 19$ & $2 \mathrm{OH}^{*}=\mathrm{H}_{2} \mathrm{O} *+\mathrm{O}^{*}$ & 0.69 & $2.24 \mathrm{E}+13$ & 0.71 & $2.24 \mathrm{E}+13$ \\
\hline M1 & $\left.\mathrm{CO}^{*}=\mathrm{CO}(\mathrm{g})\right)^{*}$ & 1.45 & $5.66 \mathrm{E}+18$ & 0.00 & $1.01 \mathrm{E}+03$ \\
\hline M2 & $\mathrm{CO}_{2}(\mathrm{~g})^{+*}=\mathrm{CO}_{2} *$ & 0.00 & $8.08 \mathrm{E}+02$ & 0.84 & $2.20 \mathrm{E}+19$ \\
\hline M3 & $\mathrm{H}_{2} \mathrm{O}^{*}=\mathrm{H}_{2} \mathrm{O}(\mathrm{g})^{+*}$ & 0.81 & $1.25 \mathrm{E}+17$ & 0.00 & $1.26 \mathrm{E}+03$ \\
\hline M4 & $\mathrm{CH}_{4}(\mathrm{~g})^{+*}=\mathrm{CH}_{4} *$ & 0.00 & $1.34 \mathrm{E}+03$ & 0.19 & $9.85 \mathrm{E}+16$ \\
\hline
\end{tabular}


Table $\mathrm{S8} \mathrm{CO}_{2} *$ activation energy barrier under different pre-adsorbed $\mathrm{O}^{*}$ coverage on $\mathrm{MoNi}_{4}(001)$.

\begin{tabular}{cc}
\hline $\mathrm{O}^{*}$ coverage $(\mathrm{ML})$ & $E_{a}(\mathrm{eV})$ \\
\hline 0 & 0.28 \\
0.2 & 0.37 \\
0.3 & 0.41 \\
0.5 & 0.53 \\
\hline
\end{tabular}

Note: Zero point energy (ZPE) and entropic contributions are not included.

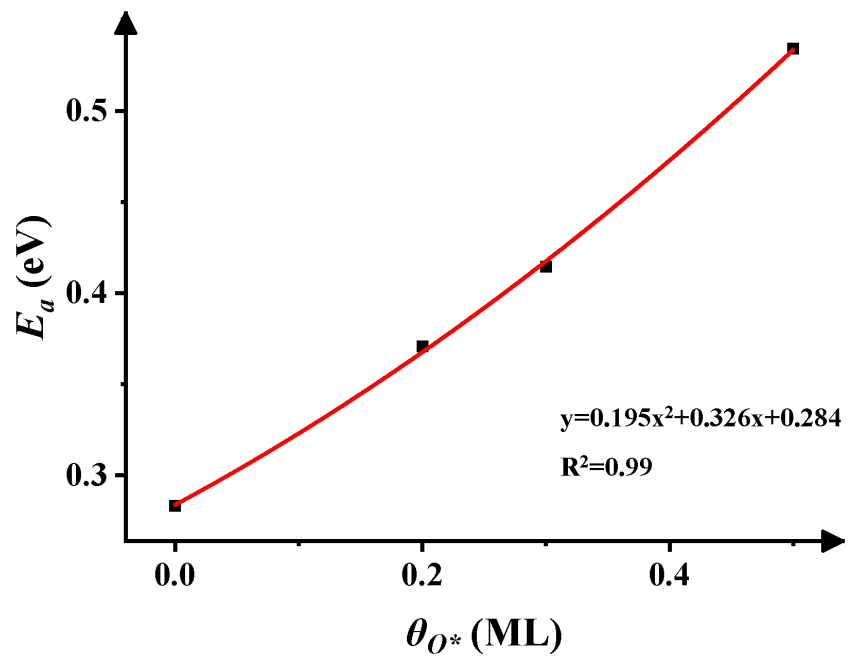

Fig. S19 Fitting curve of pre-adsorbed $\mathrm{O} *$ coverage and $\mathrm{CO}_{2} *$ activation energy barrier on $\mathrm{MoNi}_{4}(001)$.
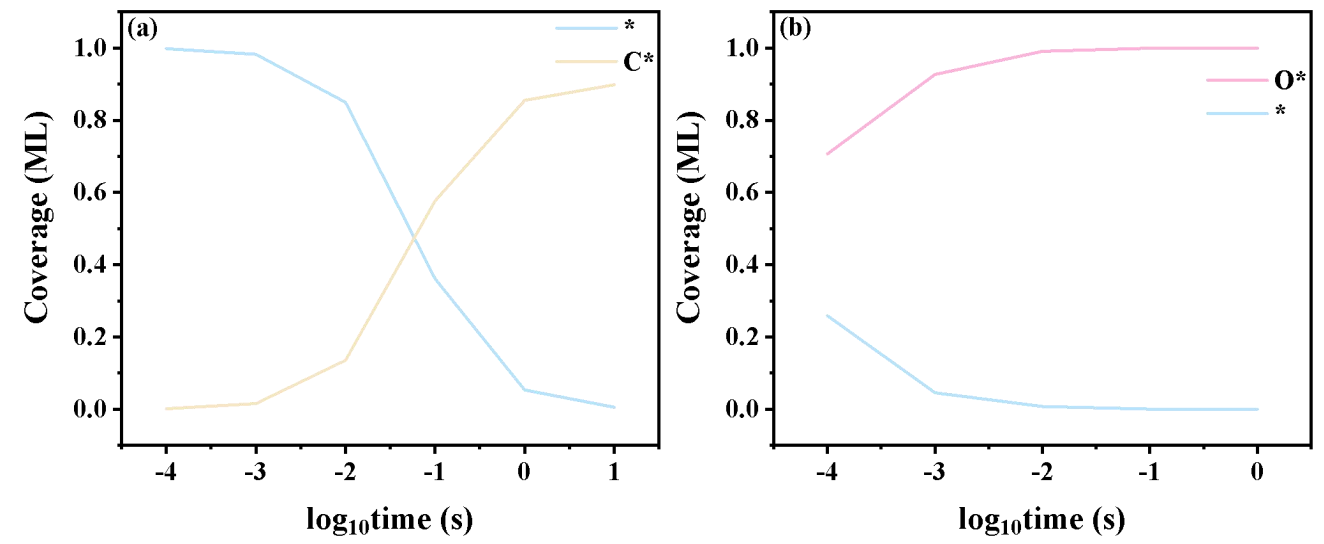

Fig. S20 The variation of the coverage of the main species over time on $\mathrm{Ni}(111)$ (a) and $\mathrm{MoNi}_{4}(001)$ (b) at 1073.15 $\mathrm{K}$ and 1 bar. 
Table S9 Under kinetic limitations, the reaction rate of the elementary reactions on Ni(111) from the microkinetic simulation at $1073.15 \mathrm{~K}$ and 1 bar.

\begin{tabular}{|c|c|c|}
\hline Label & Elementary reaction & Reaction rate $\left(\mathrm{s}^{-1}\right)$ \\
\hline $\mathrm{R} 1$ & $\mathrm{CH}_{4} *+*=\mathrm{CH}_{3} *+\mathrm{H}^{*}$ & $1.80 \mathrm{E}+01$ \\
\hline $\mathrm{R} 2$ & $\mathrm{CH}_{3} *+*=\mathrm{CH}_{2} *+\mathrm{H}^{*}$ & $1.80 \mathrm{E}+01$ \\
\hline R3 & $\mathrm{CH}_{2} *+*=\mathrm{CH}^{*+}+\mathrm{H}^{*}$ & $5.62 \mathrm{E}+02$ \\
\hline R4 & $\mathrm{CH}^{*+*}=\mathrm{C}^{*+} \mathrm{H}^{*}$ & $5.45 \mathrm{E}+03$ \\
\hline R5 & $\mathrm{CO}_{2}{ }^{*+} *=\mathrm{CO}^{*+} \mathrm{O}^{*}$ & $3.54 \mathrm{E}-01$ \\
\hline R6 & $\mathrm{CO}_{2}{ }^{*}+\mathrm{H}^{*}=\mathrm{COOH}^{*+*}$ & $1.82 \mathrm{E}-06$ \\
\hline $\mathrm{R} 7$ & $\mathrm{COOH}^{*+} *=\mathrm{CO}^{*}+\mathrm{OH}^{*}$ & $1.65 \mathrm{E}-06$ \\
\hline $\mathrm{R} 8$ & $\mathrm{CH}_{3}{ }^{*}+\mathrm{O}^{*}=\mathrm{CH}_{3} \mathrm{O}^{*+} *$ & $3.12 \mathrm{E}-08$ \\
\hline R9 & $\mathrm{CH}_{3} \mathrm{O}^{*+*}=\mathrm{CH}_{2} \mathrm{O}^{*}+\mathrm{H}^{*}$ & $3.11 \mathrm{E}-08$ \\
\hline $\mathrm{R} 10$ & $\mathrm{CH}_{2} *+\mathrm{O}^{*}=\mathrm{CH}_{2} \mathrm{O}^{*+*}$ & $1.23 \mathrm{E}-06$ \\
\hline R11 & $\mathrm{CH}_{2} \mathrm{O}^{*+*}=\mathrm{CHO}^{*+}+\mathrm{H}^{*}$ & $1.29 \mathrm{E}-06$ \\
\hline $\mathrm{R} 12$ & $\mathrm{CH}^{*+} \mathrm{O}^{*}=\mathrm{CHO}^{*+*}$ & $1.21 \mathrm{E}-01$ \\
\hline $\mathrm{R} 13$ & $\mathrm{CHO}^{*+*}=\mathrm{CO}^{*+}+\mathrm{H}^{*}$ & $1.21 \mathrm{E}-01$ \\
\hline R14 & $\mathrm{C}^{*+\mathrm{O}^{*}=\mathrm{CO}^{*+}+}$ & $1.06 \mathrm{E}-01$ \\
\hline $\mathrm{R} 15$ & $2 \mathrm{H}^{*}=\mathrm{H}_{2}(\mathrm{~g})+2^{*}$ & $3.51 \mathrm{E}+01$ \\
\hline R16 & $\mathrm{O}^{*+}+\mathrm{H}^{*}=\mathrm{OH}^{*+*}$ & $1.16 \mathrm{E}+00$ \\
\hline $\mathrm{R} 17$ & $\mathrm{OH}^{*+}+\mathrm{H}^{*}=\mathrm{H}_{2} \mathrm{O}^{*+*}$ & $7.80 \mathrm{E}-06$ \\
\hline $\mathrm{R} 18$ & $2 \mathrm{CO}^{*}=\mathrm{CO}_{2} *+\mathrm{C}^{*}$ & $1.56 \mathrm{E}-27$ \\
\hline $\mathrm{R} 19$ & $2 \mathrm{OH}^{*}=\mathrm{H}_{2} \mathrm{O}^{*}+\mathrm{O}^{*}$ & $5.29 \mathrm{E}-07$ \\
\hline M1 & $\mathrm{CO}^{*}=\mathrm{CO}(\mathrm{g})^{+*}$ & 5.81E-01 \\
\hline M2 & $\mathrm{CO}_{2}(\mathrm{~g})+*=\mathrm{CO}_{2} *$ & $7.51 \mathrm{E}+07$ \\
\hline M3 & $\mathrm{H}_{2} \mathrm{O}^{*}=\mathrm{H}_{2} \mathrm{O}(\mathrm{g})+^{*}$ & $8.33 \mathrm{E}-06$ \\
\hline M4 & $\mathrm{CH}_{4}(\mathrm{~g})+*=\mathrm{CH}_{4} *$ & $1.25 \mathrm{E}+08$ \\
\hline
\end{tabular}


Table S10 Under kinetic limitations, the coverage of intermediate species involved in DRM on Ni(111) from the microkinetic simulation at $1073.15 \mathrm{~K}$ and 1 bar.

\begin{tabular}{ccc}
\hline Label & Species & Coverage (ML) \\
\hline 1 & $*$ & $9.98 \mathrm{E}-01$ \\
2 & $\mathrm{CH}^{*}$ & $8.18 \mathrm{E}-10$ \\
4 & $\mathrm{H}^{*}$ & $1.80 \mathrm{E}-04$ \\
5 & $\mathrm{CH}^{*}$ & $1.12 \mathrm{E}-09$ \\
6 & $\mathrm{CH}^{*}$ & $1.73 \mathrm{E}-04$ \\
7 & $\mathrm{C}^{*}$ & $1.62 \mathrm{E}-03$ \\
8 & $\mathrm{CO}^{*}$ & $7.11 \mathrm{E}-13$ \\
9 & $\mathrm{O}^{*}$ & $2.70 \mathrm{E}-05$ \\
10 & $\mathrm{COOH}^{*}$ & $1.87 \mathrm{E}-17$ \\
11 & $\mathrm{OH}^{*}$ & $7.97 \mathrm{E}-10$ \\
12 & $\mathrm{CH}_{3} \mathrm{O}^{*}$ & $1.77 \mathrm{E}-17$ \\
13 & $\mathrm{CH}_{2} \mathrm{O}^{*}$ & $1.05 \mathrm{E}-18$ \\
14 & $\mathrm{CHO}^{*}$ & $5.75 \mathrm{E}-14$ \\
15 & $\mathrm{CO}_{2}{ }^{*}$ & $7.25 \mathrm{E}-11$ \\
16 & $\mathrm{H}_{2} \mathrm{O}^{*}$ & $1.28 \mathrm{E}-20$ \\
\hline
\end{tabular}

Note: The simulation results of previous studies often show that $\mathrm{CO}^{*}$ is the species with the highest coverage on the catalyst surface because it is difficult to desorb. But in our simulation, the pressure of $\mathrm{CO}(\mathrm{g})$ is constant (always 0), so the desorption of $\mathrm{CO}^{*}$ is instant in our simulation. Therefore, the difference between the simulation results and the previous research results is understandable. 
Table S11 The reaction rate of the elementary reactions on Ni(111) from the microkinetic simulation with a simulation time of $10 \mathrm{~s}$ at $1073.15 \mathrm{~K}$ and 1 bar.

\begin{tabular}{|c|c|c|}
\hline Label & Elementary reaction & Reaction rate $\left(\mathrm{s}^{-1}\right)$ \\
\hline $\mathrm{R} 1$ & $\mathrm{CH}_{4} *+*=\mathrm{CH}_{3} *+\mathrm{H}^{*}$ & $5.88 \mathrm{E}-04$ \\
\hline $\mathrm{R} 2$ & $\mathrm{CH}_{3} *+*=\mathrm{CH}_{2} *+\mathrm{H}^{*}$ & $1.88 \mathrm{E}-02$ \\
\hline R3 & $\mathrm{CH}_{2} *+*=\mathrm{CH}^{*+}+\mathrm{H}^{*}$ & $1.72 \mathrm{E}+03$ \\
\hline R4 & $\mathrm{CH}^{*+*}=\mathrm{C}^{*+} \mathrm{H}^{*}$ & $1.73 \mathrm{E}+04$ \\
\hline R5 & $\mathrm{CO}_{2}{ }^{*+*}=\mathrm{CO}^{*}+\mathrm{O}^{*}$ & $1.16 \mathrm{E}-05$ \\
\hline R6 & $\mathrm{CO}_{2}{ }^{*+} \mathrm{H}^{*}=\mathrm{COOH}^{*+*}$ & $5.96 \mathrm{E}-11$ \\
\hline R7 & $\mathrm{COOH}^{*+*}=\mathrm{CO}^{*}+\mathrm{OH}^{*}$ & $3.19 \mathrm{E}-12$ \\
\hline $\mathrm{R} 8$ & $\mathrm{CH}_{3} *+\mathrm{O}^{*}=\mathrm{CH}_{3} \mathrm{O}^{*+} *$ & $5.22 \mathrm{E}-16$ \\
\hline R9 & $\mathrm{CH}_{3} \mathrm{O}^{*+} *=\mathrm{CH}_{2} \mathrm{O}^{*}+\mathrm{H}^{*}$ & $9.57 \mathrm{E}-16$ \\
\hline $\mathrm{R} 10$ & $\mathrm{CH}_{2} *+\mathrm{O}^{*}=\mathrm{CH}_{2} \mathrm{O}^{*+*}$ & $6.07 \mathrm{E}-11$ \\
\hline $\mathrm{R} 11$ & $\mathrm{CH}_{2} \mathrm{O}^{*+*}=\mathrm{CHO}^{*+}+\mathrm{H}^{*}$ & $6.22 \mathrm{E}-11$ \\
\hline $\mathrm{R} 12$ & $\mathrm{CH}^{*+\mathrm{O}^{*}=\mathrm{CHO}^{*+} *}$ & $6.16 \mathrm{E}-06$ \\
\hline $\mathrm{R} 13$ & $\mathrm{CHO}^{*+*}=\mathrm{CO}^{*}+\mathrm{H}^{*}$ & $6.16 \mathrm{E}-06$ \\
\hline $\mathrm{R} 14$ & $\mathrm{C}^{*+\mathrm{O}^{*}=\mathrm{CO}^{*}+*}$ & $5.42 \mathrm{E}-06$ \\
\hline R15 & $2 \mathrm{H}^{*}=\mathrm{H}_{2}(\mathrm{~g})+2^{*}$ & $1.15 \mathrm{E}-03$ \\
\hline R16 & $\mathrm{O}^{*+}+\mathrm{H}^{*}=\mathrm{OH}^{*+*}$ & $6.12 \mathrm{E}-10$ \\
\hline $\mathrm{R} 17$ & $\mathrm{OH}^{*+}+\mathrm{H}^{*}=\mathrm{H}_{2} \mathrm{O}^{*+*}$ & 4.12E-15 \\
\hline $\mathrm{R} 18$ & $2 \mathrm{CO}^{*}=\mathrm{CO}_{2} *+\mathrm{C}^{*}$ & $1.57 \mathrm{E}-35$ \\
\hline R19 & $2 \mathrm{OH}^{*}=\mathrm{H}_{2} \mathrm{O}^{*}+\mathrm{O}^{*}$ & $4.51 \mathrm{E}-21$ \\
\hline M1 & $\mathrm{CO}^{*}=\mathrm{CO}(\mathrm{g})^{+*}$ & $5.83 \mathrm{E}-05$ \\
\hline M2 & $\mathrm{CO}_{2}(\mathrm{~g})+*=\mathrm{CO}_{2} *$ & $4.30 \mathrm{E}+05$ \\
\hline M3 & $\mathrm{H}_{2} \mathrm{O}^{*}=\mathrm{H}_{2} \mathrm{O}(\mathrm{g}){ }^{*} *$ & $4.12 \mathrm{E}-15$ \\
\hline M4 & $\mathrm{CH}_{4}(\mathrm{~g})+*=\mathrm{CH}_{4} *$ & $7.14 \mathrm{E}+05$ \\
\hline
\end{tabular}


Table S12 The coverage of intermediate species involved in DRM on Ni(111) from the microkinetic simulation with a simulation time of $10 \mathrm{~s}$ at $1073.15 \mathrm{~K}$ and 1 bar.

\begin{tabular}{ccc}
\hline Label & Species & Coverage (ML) \\
\hline 1 & $*$ & $5.71 \mathrm{E}-03$ \\
2 & $\mathrm{CH}_{3}{ }^{*}$ & $1.49 \mathrm{E}-10$ \\
3 & $\mathrm{H}^{*}$ & $1.03 \mathrm{E}-06$ \\
4 & $\mathrm{CH}^{*}$ & $5.98 \mathrm{E}-07$ \\
5 & $\mathrm{CH}^{*}$ & $9.59 \mathrm{E}-02$ \\
6 & $\mathrm{C}^{*}$ & $8.98 \mathrm{E}-01$ \\
7 & $\mathrm{CO}^{*}$ & $7.12 \mathrm{E}-17$ \\
8 & $\mathrm{O}^{*}$ & $2.48 \mathrm{E}-12$ \\
9 & $\mathrm{COOH}^{*}$ & $6.31 \mathrm{E}-21$ \\
10 & $\mathrm{OH}^{*}$ & $7.36 \mathrm{E}-17$ \\
11 & $\mathrm{CH}_{3} \mathrm{O}^{*}$ & $9.50 \mathrm{E}-23$ \\
12 & $\mathrm{CH}_{2} \mathrm{O}^{*}$ & $8.80 \mathrm{E}-21$ \\
13 & $\mathrm{CHO}^{*}$ & $5.10 \mathrm{E}-16$ \\
14 & $\mathrm{CO}_{2}{ }^{*}$ & $4.15 \mathrm{E}-13$ \\
15 & $\mathrm{H}_{2} \mathrm{O}^{*}$ & $6.33 \mathrm{E}-30$ \\
16 & $\mathrm{CH}_{4}{ }^{*}$ & $1.04 \mathrm{E}-10$ \\
\hline
\end{tabular}

Note: When the simulation time reaches $10 \mathrm{~s}$, the coverage of the vacancies on the surface is almost 0 , which indicates that the reaction has been difficult to proceed. Therefore, we did not continue to extend the time to simulate. 
Table S13 Under kinetic limitations, the reaction rate of the elementary reactions on $\mathrm{MoNi}_{4}(001)$ from the microkinetic simulation without considering the impact of coverage at $1073.15 \mathrm{~K}$ and 1 bar.

\begin{tabular}{|c|c|c|}
\hline Label & Elementary reaction & Reaction rate $\left(\mathrm{s}^{-1}\right)$ \\
\hline $\mathrm{R} 1$ & $\mathrm{CH}_{4} *+*=\mathrm{CH}_{3} *+\mathrm{H}^{*}$ & $8.97 \mathrm{E}+01$ \\
\hline $\mathrm{R} 2$ & $\mathrm{CH}_{3} *+*=\mathrm{CH}_{2} *+\mathrm{H}^{*}$ & $8.96 \mathrm{E}+01$ \\
\hline R3 & $\mathrm{CH}_{2} *+*=\mathrm{CH}^{*}+\mathrm{H}^{*}$ & $1.11 \mathrm{E}+02$ \\
\hline R4 & $\mathrm{CH}^{*+*}=\mathrm{C}^{*+} \mathrm{H}^{*}$ & $5.30 \mathrm{E}+04$ \\
\hline R5 & $\mathrm{CO}_{2} *+*=\mathrm{CO}^{*}+\mathrm{O}^{*}$ & $1.84 \mathrm{E}+03$ \\
\hline R6 & $\mathrm{CO}_{2} *+\mathrm{H}^{*}=\mathrm{COOH}^{*+*}$ & $1.07 \mathrm{E}-03$ \\
\hline R7 & $\mathrm{COOH}^{*+*}=\mathrm{CO}^{*+}+\mathrm{OH}^{*}$ & $2.36 \mathrm{E}-06$ \\
\hline $\mathrm{R} 8$ & $\mathrm{CH}_{3} *+\mathrm{O}^{*}=\mathrm{CH}_{3} \mathrm{O}^{*+*}$ & $2.94 \mathrm{E}-03$ \\
\hline R9 & $\mathrm{CH}_{3} \mathrm{O}^{*+*}=\mathrm{CH}_{2} \mathrm{O}^{*}+\mathrm{H}^{*}$ & $2.94 \mathrm{E}-03$ \\
\hline $\mathrm{R} 10$ & $\mathrm{CH}_{2} *+\mathrm{O}^{*}=\mathrm{CH}_{2} \mathrm{O}^{*+*}$ & 5.82E-02 \\
\hline $\mathrm{R} 11$ & $\mathrm{CH}_{2} \mathrm{O}^{*+*}=\mathrm{CHO}^{*}+\mathrm{H}^{*}$ & 2.09E-03 \\
\hline $\mathrm{R} 12$ & $\mathrm{CH}^{*+}+\mathrm{O}^{*}=\mathrm{CHO}^{*+*}$ & $1.79 \mathrm{E}+01$ \\
\hline $\mathrm{R} 13$ & $\mathrm{CHO}^{*+*}=\mathrm{CO}^{*+}+\mathrm{H}^{*}$ & $1.65 \mathrm{E}+01$ \\
\hline $\mathrm{R} 14$ & $\mathrm{C}^{*+}+\mathrm{O}^{*}=\mathrm{CO}^{*+*}$ & $9.80 \mathrm{E}-02$ \\
\hline $\mathrm{R} 15$ & $2 \mathrm{H}^{*}=\mathrm{H}_{2}(\mathrm{~g})+2^{*}$ & $1.79 \mathrm{E}+02$ \\
\hline R16 & $\mathrm{O}^{*+}+\mathrm{H}^{*}=\mathrm{OH}^{*+*}$ & $1.42 \mathrm{E}+05$ \\
\hline $\mathrm{R} 17$ & $\mathrm{OH}^{*+}+\mathrm{H}^{*}=\mathrm{H}_{2} \mathrm{O}^{*+*}$ & 8.71E-02 \\
\hline $\mathrm{R} 18$ & $2 \mathrm{CO}^{*}=\mathrm{CO}_{2} *+\mathrm{C}^{*}$ & $3.03 \mathrm{E}-12$ \\
\hline $\mathrm{R} 19$ & $2 \mathrm{OH}^{*}=\mathrm{H}_{2} \mathrm{O}^{*}+\mathrm{O}^{*}$ & $6.07 \mathrm{E}-01$ \\
\hline M1 & $\mathrm{CO}^{*}=\mathrm{CO}(\mathrm{g})+^{*}$ & $1.86 \mathrm{E}+03$ \\
\hline M2 & $\mathrm{CO}_{2}(\mathrm{~g})+*=\mathrm{CO}_{2} *$ & $2.09 \mathrm{E}+07$ \\
\hline M3 & $\mathrm{H}_{2} \mathrm{O}^{*}=\mathrm{H}_{2} \mathrm{O}(\mathrm{g})+*$ & $6.92 \mathrm{E}-01$ \\
\hline M4 & $\mathrm{CH}_{4}(\mathrm{~g})+*=\mathrm{CH}_{4} *$ & $3.47 \mathrm{E}+07$ \\
\hline
\end{tabular}


Table S14 Under kinetic limitations, the coverage of each species on $\mathrm{MoNi}_{4}(001)$ from the microkinetic simulation without considering the impact of coverage at $1073.15 \mathrm{~K}$ and 1 bar.

\begin{tabular}{ccc}
\hline Label & Species & Coverage (ML) \\
\hline 1 & $*$ & $2.59 \mathrm{E}-01$ \\
2 & $\mathrm{CH}^{*}$ & $5.20 \mathrm{E}-08$ \\
3 & $\mathrm{H}^{*}$ & $1.66 \mathrm{E}-04$ \\
4 & $\mathrm{CH}^{*}$ & $1.86 \mathrm{E}-10$ \\
5 & $\mathrm{CH}^{*}$ & $1.37 \mathrm{E}-05$ \\
6 & $\mathrm{C}^{*}$ & $3.35 \mathrm{E}-02$ \\
7 & $\mathrm{CO}^{*}$ & $2.10 \mathrm{E}-09$ \\
8 & $\mathrm{O}^{*}$ & $7.07 \mathrm{E}-01$ \\
9 & $\mathrm{COOH}^{*}$ & $1.52 \mathrm{E}-13$ \\
10 & $\mathrm{OH}^{*}$ & $6.91 \mathrm{E}-06$ \\
11 & $\mathrm{CH}_{3} \mathrm{O}^{*}$ & $1.29 \mathrm{E}-12$ \\
12 & $\mathrm{CH}_{2} \mathrm{O}^{*}$ & $3.92 \mathrm{E}-13$ \\
13 & $\mathrm{CHO}^{*}$ & $1.90 \mathrm{E}-10$ \\
14 & $\mathrm{CO}_{2}{ }^{*}$ & $8.28 \mathrm{E}-09$ \\
15 & $\mathrm{H}_{2} \mathrm{O}^{*}$ & $3.62 \mathrm{E}-14$ \\
16 & $\mathrm{CH}_{4}{ }^{*}$ & $2.83 \mathrm{E}-09$ \\
\hline
\end{tabular}


Table S15 Under kinetic limitations, the reaction rate of the elementary reactions on $\mathrm{MoNi}_{4}(001)$ from the microkinetic simulation considering the impact of coverage at $1073.15 \mathrm{~K}$ and 1 bar.

\begin{tabular}{|c|c|c|}
\hline Label & Elementary reaction & Reaction rate $\left(\mathrm{s}^{-1}\right)$ \\
\hline $\mathrm{R} 1$ & $\mathrm{CH}_{4} *+*=\mathrm{CH}_{3} *+\mathrm{H}^{*}$ & $3.56 \mathrm{E}+02$ \\
\hline $\mathrm{R} 2$ & $\mathrm{CH}_{3} *+*=\mathrm{CH}_{2} *+\mathrm{H}^{*}$ & $3.56 \mathrm{E}+02$ \\
\hline R3 & $\mathrm{CH}_{2} *+*=\mathrm{CH}^{*}+\mathrm{H}^{*}$ & $4.29 \mathrm{E}+02$ \\
\hline R4 & $\mathrm{CH}^{*+*}=\mathrm{C}^{*+} \mathrm{H}^{*}$ & $1.81 \mathrm{E}+05$ \\
\hline R5 & $\mathrm{CO}_{2}{ }^{*+} *=\mathrm{CO}^{*}+\mathrm{O}^{*}$ & $1.11 \mathrm{E}+03$ \\
\hline R6 & $\mathrm{CO}_{2} *+\mathrm{H}^{*}=\mathrm{COOH}^{*+*}$ & 4.24E-03 \\
\hline R7 & $\mathrm{COOH}^{*+*}=\mathrm{CO}^{*}+\mathrm{OH}^{*}$ & $1.86 \mathrm{E}-05$ \\
\hline $\mathrm{R} 8$ & $\mathrm{CH}_{3} *+\mathrm{O}^{*}=\mathrm{CH}_{3} \mathrm{O}^{*+*}$ & $3.53 \mathrm{E}-03$ \\
\hline R9 & $\mathrm{CH}_{3} \mathrm{O}^{*+*}=\mathrm{CH}_{2} \mathrm{O}^{*+}+\mathrm{H}^{*}$ & $3.53 \mathrm{E}-03$ \\
\hline $\mathrm{R} 10$ & $\mathrm{CH}_{2} *+\mathrm{O}^{*}=\mathrm{CH}_{2} \mathrm{O}^{*+*}$ & $6.80 \mathrm{E}-02$ \\
\hline R11 & $\mathrm{CH}_{2} \mathrm{O}^{*+*}=\mathrm{CHO}^{*+}+\mathrm{H}^{*}$ & $2.44 \mathrm{E}-03$ \\
\hline $\mathrm{R} 12$ & $\mathrm{CH}^{*+}+\mathrm{O}^{*}=\mathrm{CHO}^{*+*}$ & $1.84 \mathrm{E}+01$ \\
\hline $\mathrm{R} 13$ & $\mathrm{CHO}^{*+} *=\mathrm{CO}^{*}+\mathrm{H}^{*}$ & $1.71 \mathrm{E}+01$ \\
\hline $\mathrm{R} 14$ & $\mathrm{C}^{*+}+\mathrm{O}^{*}=\mathrm{CO}^{*+*}$ & $1.01 \mathrm{E}-01$ \\
\hline $\mathrm{R} 15$ & $2 \mathrm{H}^{*}=\mathrm{H}_{2}(\mathrm{~g})+2^{*}$ & $7.12 \mathrm{E}+02$ \\
\hline R16 & $\mathrm{O}^{*+}+\mathrm{H}^{*}=\mathrm{OH}^{*+*}$ & $1.71 \mathrm{E}+05$ \\
\hline $\mathrm{R} 17$ & $\mathrm{OH}^{*+}+\mathrm{H}^{*}=\mathrm{H}_{2} \mathrm{O}^{*+*}$ & $1.05 \mathrm{E}-01$ \\
\hline $\mathrm{R} 18$ & $2 \mathrm{CO}^{*}=\mathrm{CO}_{2} *+\mathrm{C}^{*}$ & $1.12 \mathrm{E}-12$ \\
\hline R19 & $2 \mathrm{OH}^{*}=\mathrm{H}_{2} \mathrm{O}^{*}+\mathrm{O}^{*}$ & $2.21 \mathrm{E}-01$ \\
\hline M1 & $\mathrm{CO}^{*}=\mathrm{CO}(\mathrm{g})^{+*}$ & $1.13 \mathrm{E}+03$ \\
\hline M2 & $\mathrm{CO}_{2}(\mathrm{~g})+*=\mathrm{CO}_{2} *$ & $4.17 \mathrm{E}+07$ \\
\hline M3 & $\mathrm{H}_{2} \mathrm{O}^{*}=\mathrm{H}_{2} \mathrm{O}(\mathrm{g})+*$ & $3.23 \mathrm{E}-01$ \\
\hline M4 & $\mathrm{CH}_{4}(\mathrm{~g}){ }^{*}=\mathrm{CH}_{4} *$ & $6.92 \mathrm{E}+07$ \\
\hline
\end{tabular}


Table S16 Under kinetic limitations, the coverage of each species on $\mathrm{MoNi}_{4}(001)$ from the microkinetic simulation considering the impact of coverage at $1073.15 \mathrm{~K}$ and 1 bar.

\begin{tabular}{ccc}
\hline Label & Species & Coverage (ML) \\
\hline 1 & $*$ & $5.16 \mathrm{E}-01$ \\
2 & $\mathrm{CH}^{*}$ & $1.04 \mathrm{E}-07$ \\
3 & $\mathrm{H}^{*}$ & $3.31 \mathrm{E}-04$ \\
4 & $\mathrm{CH}^{*}$ & $3.61 \mathrm{E}-10$ \\
5 & $\mathrm{CH}^{*}$ & $2.34 \mathrm{E}-05$ \\
6 & $\mathrm{C}^{*}$ & $5.72 \mathrm{E}-02$ \\
7 & $\mathrm{CO}^{*}$ & $1.27 \mathrm{E}-09$ \\
8 & $\mathrm{O}^{*}$ & $4.26 \mathrm{E}-01$ \\
9 & $\mathrm{COOH}^{*}$ & $5.98 \mathrm{E}-13$ \\
10 & $\mathrm{OH}^{*}$ & $4.17 \mathrm{E}-06$ \\
11 & $\mathrm{CH}_{3} \mathrm{O}^{*}$ & $7.76 \mathrm{E}-13$ \\
12 & $\mathrm{CH}_{2} \mathrm{O}^{*}$ & $2.30 \mathrm{E}-13$ \\
13 & $\mathrm{CHO}^{*}$ & $9.80 \mathrm{E}-11$ \\
14 & $\mathrm{CO}_{2}{ }^{*}$ & $1.65 \mathrm{E}-08$ \\
15 & $\mathrm{H}_{2} \mathrm{O}^{*}$ & $1.69 \mathrm{E}-14$ \\
16 & $\mathrm{CH}_{4}{ }^{*}$ & $5.64 \mathrm{E}-09$ \\
\hline
\end{tabular}


Table S17 Under thermodynamic limitations, the reaction rate of the elementary reactions on $\mathrm{MoNi}_{4}(001)$ from the microkinetic simulation without considering the impact of coverage at $1073.15 \mathrm{~K}$ and 1 bar.

\begin{tabular}{|c|c|c|}
\hline Label & Elementary reaction & Reaction rate $\left(\mathrm{s}^{-1}\right)$ \\
\hline $\mathrm{R} 1$ & $\mathrm{CH}_{4} *+*=\mathrm{CH}_{3} *+\mathrm{H}^{*}$ & $2.35 \mathrm{E}-10$ \\
\hline $\mathrm{R} 2$ & $\mathrm{CH}_{3} *+*=\mathrm{CH}_{2} *+\mathrm{H}^{*}$ & 7.91E-12 \\
\hline R3 & $\mathrm{CH}_{2}{ }^{*}+*=\mathrm{CH}^{*}+\mathrm{H}^{*}$ & $1.36 \mathrm{E}-11$ \\
\hline R4 & $\mathrm{CH}^{*+*}=\mathrm{C}^{*+}+\mathrm{H}^{*}$ & $1.11 \mathrm{E}-13$ \\
\hline R5 & $\mathrm{CO}_{2} *+*=\mathrm{CO}^{*}+\mathrm{O}^{*}$ & 4.82E-09 \\
\hline R6 & $\mathrm{CO}_{2}{ }^{*}+\mathrm{H}^{*}=\mathrm{COOH}^{*+*}$ & 8.37E-18 \\
\hline R7 & $\mathrm{COOH}^{*+*}=\mathrm{CO}^{*+}+\mathrm{OH}^{*}$ & $3.13 \mathrm{E}-26$ \\
\hline $\mathrm{R} 8$ & $\mathrm{CH}_{3} *+\mathrm{O}^{*}=\mathrm{CH}_{3} \mathrm{O}^{*}+*$ & $2.27 \mathrm{E}-10$ \\
\hline R9 & $\mathrm{CH}_{3} \mathrm{O}^{*+} *=\mathrm{CH}_{2} \mathrm{O}^{*}+\mathrm{H}^{*}$ & $2.27 \mathrm{E}-10$ \\
\hline $\mathrm{R} 10$ & $\mathrm{CH}_{2} *+\mathrm{O}^{*}=\mathrm{CH}_{2} \mathrm{O}^{*+*}$ & $6.24 \mathrm{E}-09$ \\
\hline R11 & $\mathrm{CH}_{2} \mathrm{O}^{*}+*=\mathrm{CHO}^{*}+\mathrm{H}^{*}$ & $2.21 \mathrm{E}-10$ \\
\hline $\mathrm{R} 12$ & $\mathrm{CH}^{*+}+\mathrm{O}^{*}=\mathrm{CHO}^{*+*}$ & $3.26 \mathrm{E}-11$ \\
\hline $\mathrm{R} 13$ & $\mathrm{CHO}^{*+*}=\mathrm{CO}^{*+}+\mathrm{H}^{*}$ & $2.35 \mathrm{E}-10$ \\
\hline $\mathrm{R} 14$ & $\mathrm{C}^{*+}+\mathrm{O}^{*}=\mathrm{CO}^{*+*}$ & $1.11 \mathrm{E}-13$ \\
\hline R15 & $2 \mathrm{H}^{*}=\mathrm{H}_{2}(\mathrm{~g})+2^{*}$ & $4.21 \mathrm{E}-15$ \\
\hline R16 & $\mathrm{O}^{*+}+\mathrm{H}^{*}=\mathrm{OH}^{*+*}$ & $9.74 \mathrm{E}-04$ \\
\hline $\mathrm{R} 17$ & $\mathrm{OH}^{*+}+\mathrm{H}^{*}=\mathrm{H}_{2} \mathrm{O}^{*+*}$ & $1.73 \mathrm{E}-12$ \\
\hline $\mathrm{R} 18$ & $2 \mathrm{CO}^{*}=\mathrm{CO}_{2} *+\mathrm{C}^{*}$ & $2.24 \mathrm{E}-35$ \\
\hline R19 & $2 \mathrm{OH}^{*}=\mathrm{H}_{2} \mathrm{O}^{*}+\mathrm{O}^{*}$ & $1.03 \mathrm{E}-05$ \\
\hline M1 & $\mathrm{CO}^{*}=\mathrm{CO}(\mathrm{g})^{+*}$ & $5.05 \mathrm{E}-09$ \\
\hline M2 & $\mathrm{CO}_{2}(\mathrm{~g})+*=\mathrm{CO}_{2} *$ & $3.38 \mathrm{E}+01$ \\
\hline M3 & $\mathrm{H}_{2} \mathrm{O}^{*}=\mathrm{H}_{2} \mathrm{O}(\mathrm{g})+^{*}$ & 4.04E-10 \\
\hline M4 & $\mathrm{CH}_{4}(\mathrm{~g})+*=\mathrm{CH}_{4} *$ & $5.62 \mathrm{E}+01$ \\
\hline
\end{tabular}


Table S18 Under thermodynamic limitations, the coverage of each species on $\mathrm{MoNi}_{4}(001)$ from the microkinetic simulation without considering the impact of coverage at $1073.15 \mathrm{~K}$ and 1 bar.

\begin{tabular}{ccc}
\hline Label & Species & Coverage (ML) \\
\hline 1 & $*$ & $4.19 \mathrm{E}-07$ \\
2 & $\mathrm{CH}^{*}$ & $2.84 \mathrm{E}-15$ \\
3 & $\mathrm{H}^{*}$ & $8.05 \mathrm{E}-13$ \\
4 & $\mathrm{CH}^{*}$ & $1.41 \mathrm{E}-17$ \\
5 & $\mathrm{CH}^{*}$ & $1.76 \mathrm{E}-17$ \\
6 & $\mathrm{C}^{*}$ & $2.67 \mathrm{E}-14$ \\
7 & $\mathrm{CO}^{*}$ & $5.70 \mathrm{E}-21$ \\
8 & $\mathrm{O}^{*}$ & $1.00 \mathrm{E}+00$ \\
9 & $\mathrm{COOH}^{*}$ & $1.24 \mathrm{E}-27$ \\
10 & $\mathrm{OH}^{*}$ & $2.84 \mathrm{E}-08$ \\
11 & $\mathrm{CH}_{3} \mathrm{O}^{*}$ & $6.15 \mathrm{E}-14$ \\
12 & $\mathrm{CH}_{2} \mathrm{O}^{*}$ & $2.56 \mathrm{E}-14$ \\
13 & $\mathrm{CHO}^{*}$ & $1.66 \mathrm{E}-15$ \\
14 & $\mathrm{CO}_{2}{ }^{*}$ & $1.34 \mathrm{E}-14$ \\
15 & $\mathrm{H}_{2} \mathrm{O}^{*}$ & $2.12 \mathrm{E}-23$ \\
16 & $\mathrm{CH}_{4}{ }^{*}$ & $4.58 \mathrm{E}-15$ \\
\hline
\end{tabular}


Table S19 Under thermodynamic limitations, the reaction rate of the elementary reactions on $\mathrm{MoNi}_{4}(001)$ from the microkinetic simulation considering the impact of coverage at $1073.15 \mathrm{~K}$ and 1 bar.

\begin{tabular}{|c|c|c|}
\hline Label & Elementary reaction & Reaction rate $\left(\mathrm{s}^{-1}\right)$ \\
\hline $\mathrm{R} 1$ & $\mathrm{CH}_{4} *+*=\mathrm{CH}_{3} *+\mathrm{H}^{*}$ & $6.69 \mathrm{E}+01$ \\
\hline $\mathrm{R} 2$ & $\mathrm{CH}_{3} *+*=\mathrm{CH}_{2} *+\mathrm{H}^{*}$ & $6.68 \mathrm{E}+01$ \\
\hline R3 & $\mathrm{CH}_{2}{ }^{*}+*=\mathrm{CH}^{*}+\mathrm{H}^{*}$ & $1.51 \mathrm{E}+02$ \\
\hline R4 & $\mathrm{CH}^{*+*}=\mathrm{C}^{*+} \mathrm{H}^{*}$ & $2.09 \mathrm{E}+05$ \\
\hline R5 & $\mathrm{CO}_{2} *+*=\mathrm{CO}^{*}+\mathrm{O}^{*}$ & $6.73 \mathrm{E}+01$ \\
\hline R6 & $\mathrm{CO}_{2} *+\mathrm{H}^{*}=\mathrm{COOH}^{*+*}$ & 7.95E-04 \\
\hline R7 & $\mathrm{COOH}^{*+*}=\mathrm{CO}^{*+}+\mathrm{OH}^{*}$ & $1.51 \mathrm{E}-06$ \\
\hline R8 & $\mathrm{CH}_{3} *+\mathrm{O}^{*}=\mathrm{CH}_{3} \mathrm{O}^{*}+*$ & $2.24 \mathrm{E}-03$ \\
\hline R9 & $\mathrm{CH}_{3} \mathrm{O}^{*+*}=\mathrm{CH}_{2} \mathrm{O}^{*}+\mathrm{H}^{*}$ & $2.23 \mathrm{E}-03$ \\
\hline $\mathrm{R} 10$ & $\mathrm{CH}_{2} *+\mathrm{O}^{*}=\mathrm{CH}_{2} \mathrm{O}^{*}+*$ & $8.08 \mathrm{E}-02$ \\
\hline R11 & $\mathrm{CH}_{2} \mathrm{O}^{*+} *=\mathrm{CHO}^{*}+\mathrm{H}^{*}$ & $2.84 \mathrm{E}-03$ \\
\hline $\mathrm{R} 12$ & $\mathrm{CH}^{*}+\mathrm{O}^{*}=\mathrm{CHO}^{*+*}$ & $7.18 \mathrm{E}+01$ \\
\hline $\mathrm{R} 13$ & $\mathrm{CHO}^{*+} *=\mathrm{CO}^{*+}+\mathrm{H}^{*}$ & $6.64 \mathrm{E}+01$ \\
\hline $\mathrm{R} 14$ & $\mathrm{C}^{*+}+\mathrm{O}^{*}=\mathrm{CO}^{*+*}$ & $3.95 \mathrm{E}-01$ \\
\hline $\mathrm{R} 15$ & $2 \mathrm{H}^{*}=\mathrm{H}_{2}(\mathrm{~g})+2^{*}$ & $1.33 \mathrm{E}+02$ \\
\hline R16 & $\mathrm{O}^{*+}+\mathrm{H}^{*}=\mathrm{OH}^{*+*}$ & $1.08 \mathrm{E}+05$ \\
\hline $\mathrm{R} 17$ & $\mathrm{OH}^{*+}+\mathrm{H}^{*}=\mathrm{H}_{2} \mathrm{O}^{*+*}$ & $6.60 \mathrm{E}-02$ \\
\hline $\mathrm{R} 18$ & $2 \mathrm{CO}^{*}=\mathrm{CO}_{2} *+\mathrm{C}^{*}$ & $1.58 \mathrm{E}-14$ \\
\hline R19 & $2 \mathrm{OH}^{*}=\mathrm{H}_{2} \mathrm{O}^{*}+\mathrm{O}^{*}$ & 4.70E-01 \\
\hline M1 & $\mathrm{CO}^{*}=\mathrm{CO}(\mathrm{g})+^{*}$ & $1.34 \mathrm{E}+02$ \\
\hline M2 & $\mathrm{CO}_{2}(\mathrm{~g})+*=\mathrm{CO}_{2} *$ & $1.81 \mathrm{E}+07$ \\
\hline M3 & $\mathrm{H}_{2} \mathrm{O}^{*}=\mathrm{H}_{2} \mathrm{O}(\mathrm{g})+^{*}$ & $5.34 \mathrm{E}-01$ \\
\hline M4 & $\mathrm{CH}_{4}(\mathrm{~g}){ }^{*}=\mathrm{CH}_{4} *$ & $3.00 \mathrm{E}+07$ \\
\hline
\end{tabular}


Table S20 Under thermodynamic limitations, the coverage of each species on $\mathrm{MoNi}_{4}(001)$ from the microkinetic simulation considering the impact of coverage at $1073.15 \mathrm{~K}$ and 1 bar.

\begin{tabular}{ccc}
\hline Label & Species & Coverage (ML) \\
\hline 1 & $*$ & $2.24 \mathrm{E}-01$ \\
2 & $\mathrm{CH}^{*}$ & $4.49 \mathrm{E}-08$ \\
3 & $\mathrm{H}^{*}$ & $1.43 \mathrm{E}-04$ \\
4 & $\mathrm{CH}^{*}$ & $2.93 \mathrm{E}-10$ \\
5 & $\mathrm{CH}^{*}$ & $6.23 \mathrm{E}-05$ \\
6 & $\mathrm{C}^{*}$ & $1.53 \mathrm{E}-01$ \\
7 & $\mathrm{CO}^{*}$ & $1.52 \mathrm{E}-10$ \\
8 & $\mathrm{O}^{*}$ & $6.23 \mathrm{E}-01$ \\
9 & $\mathrm{COOH}^{*}$ & $1.12 \mathrm{E}-13$ \\
10 & $\mathrm{OH}^{*}$ & $6.08 \mathrm{E}-06$ \\
11 & $\mathrm{CH}_{3} \mathrm{O}^{*}$ & $1.14 \mathrm{E}-12$ \\
12 & $\mathrm{CH}_{2} \mathrm{O}^{*}$ & $6.16 \mathrm{E}-13$ \\
13 & $\mathrm{CHO}^{*}$ & $8.81 \mathrm{E}-10$ \\
14 & $\mathrm{CO}_{2}{ }^{*}$ & $7.15 \mathrm{E}-09$ \\
15 & $\mathrm{H}_{2} \mathrm{O}^{*}$ & $2.80 \mathrm{E}-14$ \\
16 & $\mathrm{CH}_{4}{ }^{*}$ & $2.44 \mathrm{E}-09$ \\
\hline
\end{tabular}




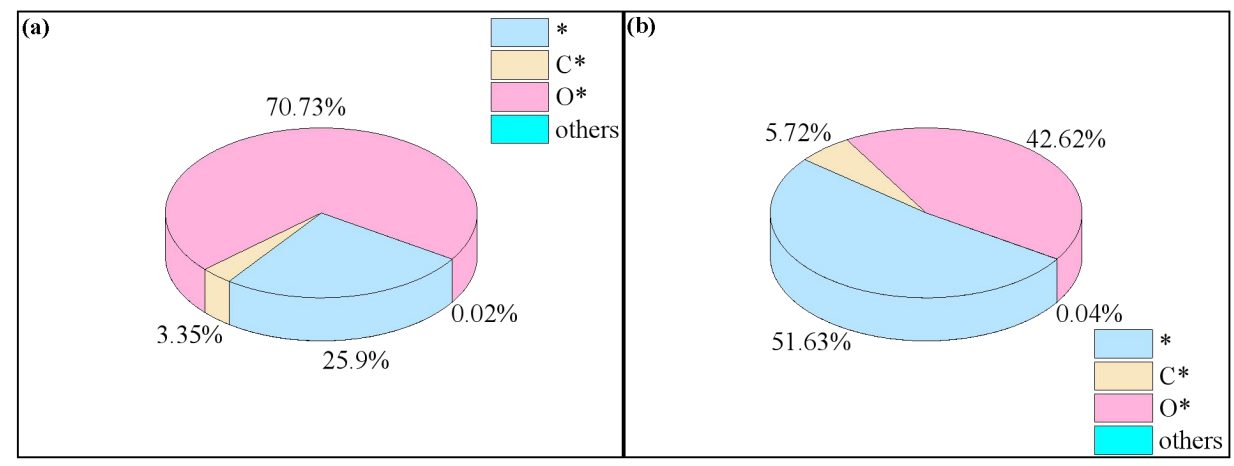

Fig. S21 Under kinetic limitations, coverage distributions on $\mathrm{MoNi}_{4}(001)$ from simulations without considering the impact of coverage (a) and simulations considering the impact of coverage (b).

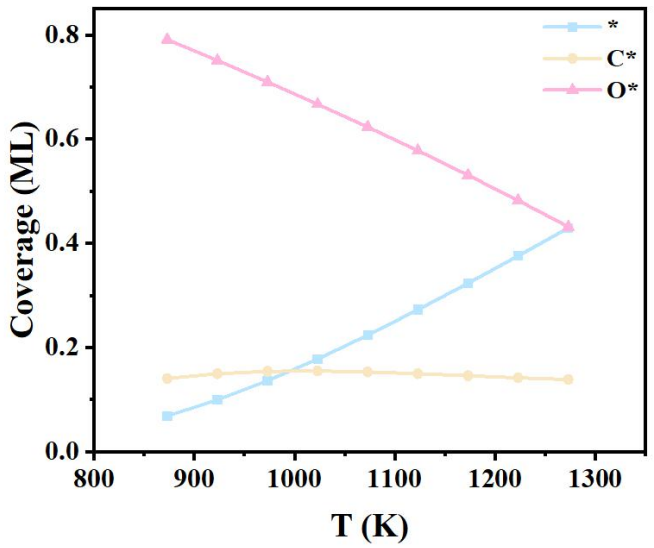

Fig. S22 Under thermodynamic limitations, the change trend of main species coverage with temperature on $\mathrm{MoNi}_{4}(001)$ from simulations considering the impact of coverage in the temperature range of 873.15-1273.15 K.

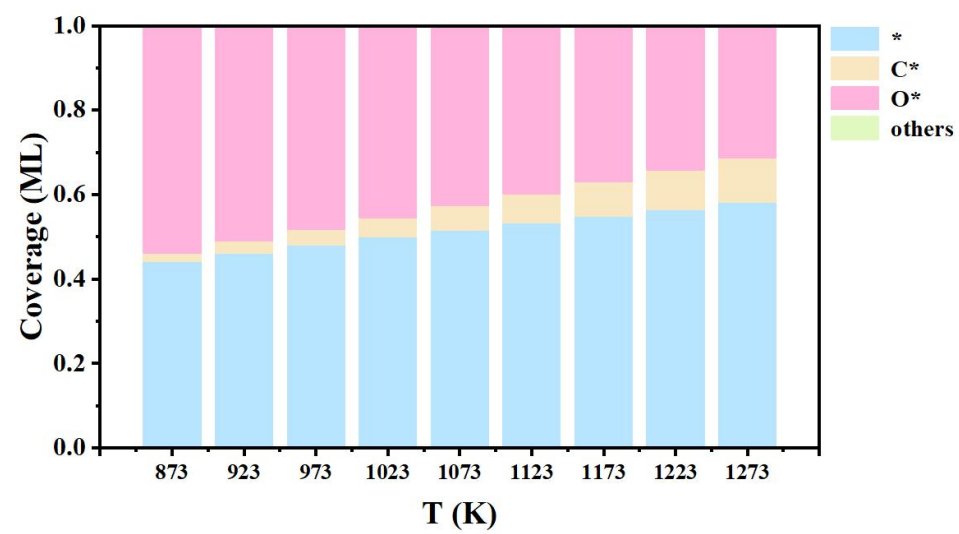

Fig. S23 Under kinetic limitations, the variation of coverage distributions with temperature on $\mathrm{MoNi}_{4}(001)$ from simulations considering the impact of coverage in the temperature range of 873.15-1273.15 K. 


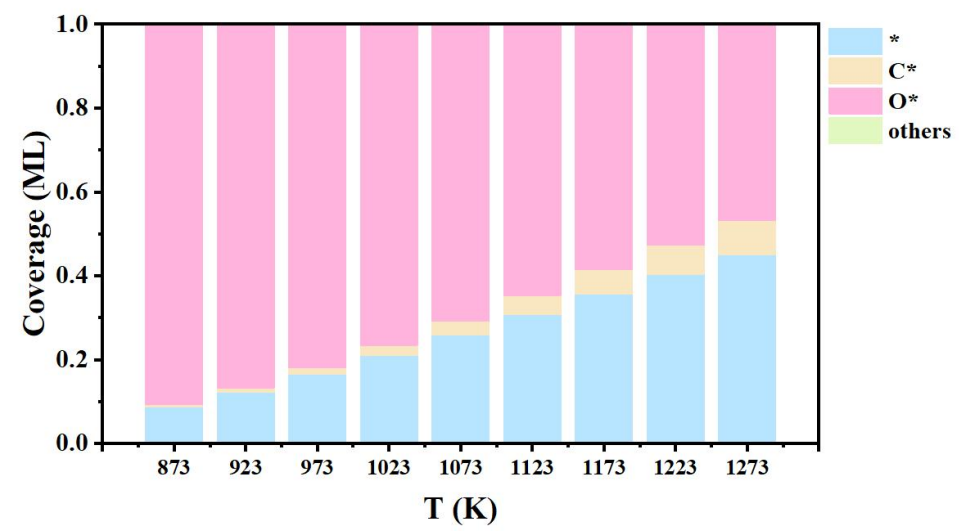

Fig. S24 Under kinetic limitations, the variation of coverage distributions with temperature on $\mathrm{MoNi}_{4}(001)$ from simulations without considering the impact of coverage in the temperature range of 873.15-1273.15 K.

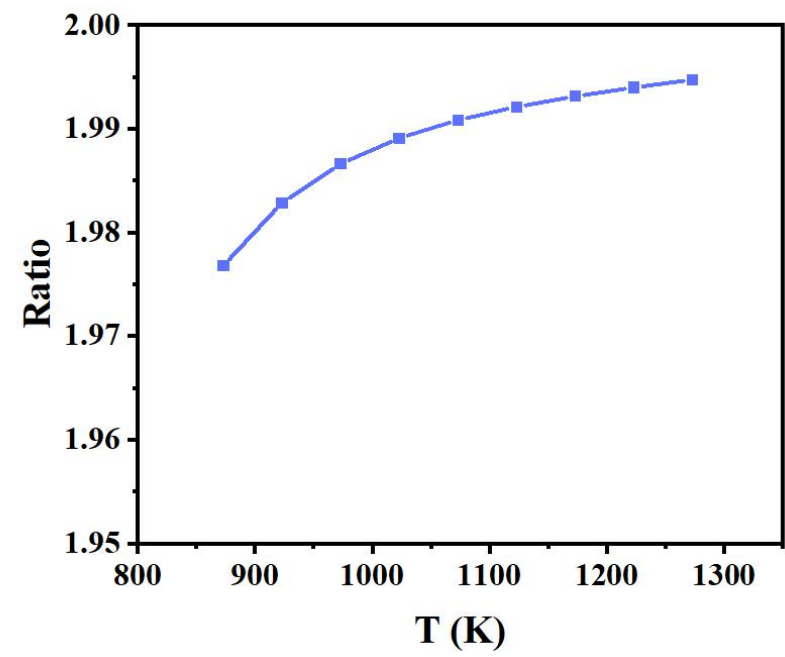

Fig. S25 Under thermodynamic limitations, the change of the ratio of $\mathrm{CO}$ generation rate to $\mathrm{CO}_{2}$ activation rate with temperature on $\mathrm{MoNi}_{4}(001)$ from simulations considering the impact of coverage in the temperature range of $873.15-1273.15 \mathrm{~K}$.

\section{References}

(1) Kresse, G.; Hafner, J. Ab initio molecular dynamics for liquid metals. Phys Rev B Condens Matter 1993, 47, 558-561.

(2) Kresse, G.; Furthmüller, J. Efficiency of ab-initio total energy calculations for metals and semiconductors using a plane-wave basis set. Computational materials science 1996, 6, 15-50.

(3) Kresse, G.; Furthmüller, J. Efficient iterative schemes for ab initio total-energy calculations using a plane-wave basis set. $J$ Physical review $B$ 1996, 54, 11169.

(4) Blochl, P.E. Projector augmented-wave method. Phys Rev B Condens Matter 1994, 50, 17953-17979.

(5) Kresse, G.; Joubert, D. From ultrasoft pseudopotentials to the projector augmented-wave method. Physical Review B 1999, 59 1758-1775.

(6) Perdew, J.P.; Burke, K.; Ernzerhof, M. Generalized Gradient Approximation Made Simple. Physical Review Letters 1996, 77, 3865-3868.

(7) Perdew, J.P.; Wang, Y. Accurate and simple analytic representation of the electron-gas correlation energy. Phys Rev B 
Condens Matter 1992, 45, 13244-13249.

(8) Monkhorst, H.J.; Pack, J.D. On Special Points for Brillouin Zone Integrations. Physical review. B, Condensed matter 1976, 13, 5188-5192.

(9) Kresse, G.; Hafner, J. First-principles study of the adsorption of atomic H on Ni (111),(100) and (110). Surface science 2000, 459, 287-302.

(10) Grimme, S.; Antony, J.; Ehrlich, S.; Krieg, H. A consistent and accurate ab initio parametrization of density functional dispersion correction (DFT-D) for the 94 elements H-Pu. J Chem Phys 2010, 132, 154104.

(11) Grimme, S.; Ehrlich, S.; Goerigk, L. Effect of the damping function in dispersion corrected density functional theory. journal of computational chemistry 2011, 32, 1456-1465.

(12) Behler, J. Perspective: Machine learning potentials for atomistic simulations. J Chem Phys 2016, 145, 170901.

(13) Harker, D. The Crystal Structure of Ni4Mo. The Journal of Chemical Physics 1944, 12, 315-317.

(14) Lide, D.R., CRC handbook of chemistry and physics, CRC press, 2004.

(15) Campbell, C.T.; Sellers, J.R.V. Correction to "The Entropies of Adsorbed Molecules". Journal of the American Chemical Society 2013, 135, 13998-13998.

(16) Cortright, R.D.; Dumesic, J.A., Kinetics of heterogeneous catalytic reactions: Analysis of reaction schemes, in: Advances in Catalysis, Academic Press, 2001, 46, 161-264.

(17) Filot, I.A.; Broos, R.J.; van Rijn, J.P.; van Heugten, G.J.; van Santen, R.A.; Hensen, E.J. First-principles-based microkinetics simulations of synthesis gas conversion on a stepped rhodium surface. ACS Catalysis 2015, 5, 5453-5467.

(18) Hindmarsh, A.C. ODEPACK, a systematized collection of ODE solvers. Scientific computing 1983, 55-64.

(19) Qi, K.; Zhao, J.-M.; Wang, G.-C. A density functional theory study of ethylene hydrogenation on MgO-and $\gamma-\mathrm{Al}_{2} \mathrm{O}_{3}$-supported carbon-containing $\mathrm{Ir}_{4}$ clusters. Physical Chemistry Chemical Physics 2015, 17, 4899-4908.

(20) Yao, Z.; Guo, C.; Mao, Y.; Hu, P. Quantitative Determination of C-C Coupling Mechanisms and Detailed Analyses on the Activity and Selectivity for Fischer-Tropsch Synthesis on Co(0001): Microkinetic Modeling with Coverage Effects. ACS Catalysis 2019, 9, 5957-5973.

(21) Zhu, Y.-A.; Chen, D.; Zhou, X.-G.; Yuan, W.-K. DFT studies of dry reforming of methane on Ni catalyst. Catalysis Today 2009, 148, 260-267. 